\title{
Top quark mass measurement using the template method in the lepton + jets channel at CDF II
}

A. Abulencia, ${ }^{23}$ D. Acosta, ${ }^{17}$ J. Adelman, ${ }^{13}$ T. Affolder, ${ }^{10}$ T. Akimoto, ${ }^{53}$ M. G. Albrow,${ }^{16}$ D. Ambrose,${ }^{16}$ S. Amerio, ${ }^{42}$ D. Amidei, ${ }^{33}$ A. Anastassov, ${ }^{50}$ K. Anikeev, ${ }^{16}$ A. Annovi, ${ }^{44}$ J. Antos, ${ }^{1}$ M. Aoki, ${ }^{53}$ G. Apollinari, ${ }^{16}$ J.-F. Arguin, ${ }^{32}$ T. Arisawa, ${ }^{55}$ A. Artikov, ${ }^{14}$ W. Ashmanskas, ${ }^{16}$ A. Attal, ${ }^{8}$ F. Azfar, ${ }^{41}$ P. Azzi-Bacchetta, ${ }^{42}$ P. Azzurri, ${ }^{44}$ N. Bacchetta, ${ }^{42}$ H. Bachacou, ${ }^{28}$ W. Badgett, ${ }^{16}$ A. Barbaro-Galtieri, ${ }^{28}$ V. E. Barnes, ${ }^{46}$ B. A. Barnett,${ }^{24}$ S. Baroiant,${ }^{7}$ V. Bartsch, ${ }^{30}$ G. Bauer, ${ }^{31}$ F. Bedeschi, ${ }^{44}$ S. Behari, ${ }^{24}$ S. Belforte,${ }^{52}$ G. Bellettini, ${ }^{44}$ J. Bellinger, ${ }^{57}$ A. Belloni, ${ }^{31}$ E. Ben-Haim, ${ }^{16}$ D. Benjamin, ${ }^{15}$ A. Beretvas, ${ }^{16}$ J. Beringer, ${ }^{28}$ T. Berry, ${ }^{29}$ A. Bhatti, ${ }^{48}$ M. Binkley, ${ }^{16}$ D. Bisello, ${ }^{42}$ M. Bishai, ${ }^{16}$ R. E. Blair, ${ }^{2}$ C. Blocker, ${ }^{6}$ K. Bloom ${ }^{33}$ B. Blumenfeld, ${ }^{24}$ A. Bocci, ${ }^{48}$ A. Bodek,${ }^{47}$ V. Boisvert, ${ }^{47}$ G. Bolla ${ }^{46}$ A. Bolshov, ${ }^{31}$ D. Bortoletto, ${ }^{46}$

J. Boudreau, ${ }^{45}$ S. Bourov, ${ }^{16}$ A. Boveia,${ }^{10}$ B. Brau,${ }^{10}$ C. Bromberg, ${ }^{34}$ E. Brubaker, ${ }^{13}$ J. Budagov, ${ }^{14}$ H. S. Budd,${ }^{47}$ S. Budd,${ }^{23}$ K. Burkett, ${ }^{16}$ G. Busetto, ${ }^{42}$ P. Bussey, ${ }^{20}$ K. L. Byrum, ${ }^{2}$ S. Cabrera, ${ }^{15}$ M. Campanelli, ${ }^{19}$ M. Campbell, ${ }^{33}$ F. Canelli, ${ }^{8}$

A. Canepa,${ }^{46}$ D. Carlsmith, ${ }^{57}$ R. Carosi, ${ }^{44}$ S. Carron,,${ }^{15}$ M. Casarsa,${ }^{52}$ A. Castro, ${ }^{5}$ P. Catastini, ${ }^{44}$ D. Cauz,${ }^{52}$

M. Cavalli-Sforza, ${ }^{3}$ A. Cerri, ${ }^{28}$ L. Cerrito, ${ }^{41}$ S. H. Chang, ${ }^{27}$ J. Chapman, ${ }^{33}$ Y. C. Chen, ${ }^{1}$ M. Chertok, ${ }^{7}$ G. Chiarelli, ${ }^{44}$ G. Chlachidze, ${ }^{14}$ F. Chlebana, ${ }^{16}$ I. Cho, ${ }^{27}$ K. Cho, ${ }^{27}$ D. Chokheli, ${ }^{14}$ J. P. Chou, ${ }^{21}$ P. H. Chu, ${ }^{23}$ S. H. Chuang, ${ }^{57}$ K. Chung, ${ }^{12}$ W. H. Chung, ${ }^{57}$ Y. S. Chung, ${ }^{47}$ M. Ciljak, ${ }^{44}$ C. I. Ciobanu, ${ }^{23}$ M. A. Ciocci, ${ }^{44}$ A. Clark, ${ }^{19}$ D. Clark, ${ }^{6}$ M. Coca, ${ }^{15}$ A. Connolly, ${ }^{28}$ M. E. Convery, ${ }^{48}$ J. Conway, ${ }^{7}$ B. Cooper,${ }^{30}$ K. Copic,${ }^{33}$ M. Cordelli, ${ }^{18}$ G. Cortiana, ${ }^{42}$ A. Cruz, ${ }^{17}$ J. Cuevas, ${ }^{11}$ R. Culbertson, ${ }^{16}$ C. Currat ${ }^{28}$ D. Cyr, ${ }^{57}$ S. DaRonco, ${ }^{42}$ S. D'Auria, ${ }^{20}$ M. D'onofrio, ${ }^{19}$ D. Dagenhart, ${ }^{6}$ P. de Barbaro, ${ }^{47}$ S. De Cecco, ${ }^{49}$ A. Deisher, ${ }^{28}$ G. De Lentdecker, ${ }^{47}$ M. Dell'Orso, ${ }^{44}$ S. Demers, ${ }^{47}$ L. Demortier, ${ }^{48}$ J. Deng, ${ }^{15}$ M. Deninno, ${ }^{5}$ D. De Pedis, ${ }^{49}$ P. F. Derwent, ${ }^{16}$ C. Dionisi, ${ }^{49}$ J. Dittmann, ${ }^{4}$ P. DiTuro, ${ }^{50}$ C. Dörr, ${ }^{25}$ A. Dominguez, ${ }^{28}$ S. Donati, ${ }^{44}$ M. Donega,${ }^{19}$ P. Dong, ${ }^{8}$ J. Donini, ${ }^{42}$ T. Dorigo, ${ }^{42}$ S. Dube,${ }^{50}$ K. Ebina, ${ }^{55}$ J. Efron, ${ }^{38}$ J. Ehlers,${ }^{19}$ R. Erbacher, ${ }^{7}$ D. Errede,${ }^{23}$ S. Errede, ${ }^{23}$ R. Eusebi, ${ }^{47}$ H. C. Fang, ${ }^{28}$ S. Farrington, ${ }^{29}$ I. Fedorko, ${ }^{44}$ W. T. Fedorko, ${ }^{13}$ R. G. Feild, ${ }^{58}$ M. Feindt, ${ }^{25}$ J.P. Fernandez, ${ }^{46}$ R. Field, ${ }^{17}$ G. Flanagan, ${ }^{34}$ L. R. Flores-Castillo, ${ }^{45}$ A. Foland, ${ }^{21}$ S. Forrester, ${ }^{7}$ G. W. Foster, ${ }^{16}$ M. Franklin, ${ }^{21}$ J. C. Freeman, ${ }^{28}$ Y. Fujii, ${ }^{26}$ I. Furic,${ }^{13}$ A. Gajjar, ${ }^{29}$ M. Gallinaro, ${ }^{48}$ J. Galyardt, ${ }^{12}$ J. E. Garcia, ${ }^{44}$ M. Garcia Sciveres ${ }^{28}$ A. F. Garfinkel,${ }^{46}$ C. Gay, ${ }^{58}$ H. Gerberich, ${ }^{23}$ E. Gerchtein, ${ }^{12}$ D. Gerdes,${ }^{33}$ S. Giagu, ${ }^{49}$ P. Giannetti,${ }^{44}$ A. Gibson, ${ }^{28}$ K. Gibson, ${ }^{12}$ C. Ginsburg,${ }^{16}$ K. Giolo,${ }^{46}$ M. Giordani, ${ }^{52}$ M. Giunta, ${ }^{44}$ G. Giurgiu, ${ }^{12}$ V. Glagolev, ${ }^{14}$ D. Glenzinski, ${ }^{16} \mathrm{M}$. Gold, ${ }^{36} \mathrm{~N}$. Goldschmidt, ${ }^{33} \mathrm{~J}$. Goldstein, ${ }^{41} \mathrm{G}$. Gomez, ${ }^{11} \mathrm{G}$. Gomez-Ceballos, ${ }^{11} \mathrm{M}$. Goncharov, ${ }^{51}$ O. González, ${ }^{46}$ I. Gorelov, ${ }^{36}$ A. T. Goshaw, ${ }^{15}$ Y. Gotra, ${ }^{45}$ K. Goulianos ${ }^{48}$ A. Gresele, ${ }^{42}$ M. Griffiths, ${ }^{29}$ S. Grinstein ${ }^{21}$ C. Grosso-Pilcher, ${ }^{13}$ U. Grundler, ${ }^{23}$ J. Guimaraes da Costa, ${ }^{21}$ C. Haber,${ }^{28}$ S. R. Hahn, ${ }^{16}$ K. Hahn, ${ }^{43}$ E. Halkiadakis, ${ }^{47}$ A. Hamilton, ${ }^{32}$ B.-Y. Han, ${ }^{47}$ R. Handler,${ }^{57}$ F. Happacher,${ }^{18}$ K. Hara,${ }^{53}$ M. Hare, ${ }^{54}$ S. Harper, ${ }^{41}$ R. F. Harr,${ }^{56}$ R. M. Harris, ${ }^{16}$ K. Hatakeyama ${ }^{48}$ J. Hauser, ${ }^{8}$ C. Hays,${ }^{15}$ H. Hayward, ${ }^{29}$ A. Heijboer, ${ }^{43}$ B. Heinemann, ${ }^{29}$ J. Heinrich, ${ }^{43}$ M. Hennecke, ${ }^{25}$ M. Herndon, ${ }^{57}$ J. Heuser, ${ }^{25}$ D. Hidas, ${ }^{15}$ C. S. Hill, ${ }^{10}$ D. Hirschbuehl, ${ }^{25}$ A. Hocker,${ }^{16}$ A. Holloway,,${ }^{21}$ S. Hou, ${ }^{1}$ M. Houlden,${ }^{29}$ S.-C. Hsu, ${ }^{9}$ B. T. Huffman, ${ }^{41}$ R. E. Hughes,${ }^{38}$ J. Huston, ${ }^{34}$ K. Ikado,${ }^{55}$ J. Incandela, ${ }^{10}$ G. Introzzi, ${ }^{44}$ M. Iori, ${ }^{49}$ Y. Ishizawa, ${ }^{53}$ A. Ivanov, ${ }^{7}$ B. Iyutin, ${ }^{31}$ E. James, ${ }^{16}$ D. Jang,${ }^{50}$ B. Jayatilaka, ${ }^{33}$ D. Jeans, ${ }^{49}$ H. Jensen, ${ }^{16}$ E. J. Jeon, ${ }^{27}$ M. Jones,${ }^{46}$ K. K. Joo, ${ }^{27}$ S. Y. Jun, ${ }^{12}$ T. R. Junk, ${ }^{23}$ T. Kamon, ${ }^{51}$ J. Kang, ${ }^{33}$ M. Karagoz-Unel, ${ }^{37}$ P. E. Karchin,${ }^{56}$ Y. Kato, ${ }^{40}$ Y. Kemp,${ }^{25}$ R. Kephart, ${ }^{16}$ U. Kerzel, ${ }^{25}$ V. Khotilovich, ${ }^{51}$ B. Kilminster, ${ }^{38}$ D. H. Kim, ${ }^{27}$ H. S. Kim, ${ }^{27}$ J. E. Kim, ${ }^{27}$ M. J. Kim, ${ }^{12}$ M. S. Kim, ${ }^{27}$ S. B. Kim, ${ }^{27}$ S. H. Kim ${ }^{53}$ Y. K. Kim, ${ }^{13}$ M. Kirby, ${ }^{15}$ L. Kirsch, ${ }^{6}$ S. Klimenko, ${ }^{17}$ M. Klute, ${ }^{31}$ B. Knuteson, ${ }^{31}$ B. R. Ko, ${ }^{15}$ H. Kobayashi, ${ }^{53}$ K. Kondo, ${ }^{55}$ D. J. Kong, ${ }^{27}$ J. Konigsberg, ${ }^{17}$ K. Kordas, ${ }^{18}$ A. Korytov, ${ }^{17}$ A. V. Kotwal, ${ }^{15}$ A. Kovalev, ${ }^{43}$ J. Kraus, ${ }^{23}$ I. Kravchenko, ${ }^{31}$ M. Kreps, ${ }^{25}$ A. Kreymer, ${ }^{16}$ J. Kroll, ${ }^{43}$ N. Krumnack, ${ }^{4}$ M. Kruse, ${ }^{15}$ V. Krutelyov, ${ }^{51}$ S. E. Kuhlmann, ${ }^{2}$ Y. Kusakabe, ${ }^{55}$ S. Kwang, ${ }^{13}$ A. T. Laasanen, ${ }^{46}$ S. Lai, ${ }^{32}$ S. Lami, ${ }^{44}$ S. Lammel, ${ }^{16}$ M. Lancaster, ${ }^{30}$

R. L. Lander, ${ }^{7}$ K. Lannon, ${ }^{38}$ A. Lath, ${ }^{50}$ G. Latino, ${ }^{44}$ I. Lazzizzera, ${ }^{42}$ C. Lecci, ${ }^{25}$ T. LeCompte,${ }^{2}$ J. Lee, ${ }^{47}$ J. Lee, ${ }^{27}$ S. W. Lee, ${ }^{51}$ Y. J. Lee, ${ }^{27}$ R. Lefèvre, ${ }^{3}$ N. Leonardo, ${ }^{31}$ S. Leone, ${ }^{44}$ S. Levy, ${ }^{13}$ J. D. Lewis, ${ }^{16}$ K. Li,${ }^{58}$ C. Lin,${ }^{58}$ C. S. Lin,${ }^{16}$ M. Lindgren, ${ }^{16}$ E. Lipeles, ${ }^{9}$ T. M. Liss,${ }^{23}$ A. Lister, ${ }^{19}$ D. O. Litvintsev, ${ }^{16}$ T. Liu, ${ }^{16}$ Y. Liu, ${ }^{19}$ N. S. Lockyer, ${ }^{43}$ A. Loginov, ${ }^{35}$ M. Loreti, ${ }^{42}$ P. Loverre, ${ }^{49}$ R.-S. Lu, ${ }^{1}$ D. Lucchesi,${ }^{42}$ P. Lujan, ${ }^{28}$ P. Lukens,,${ }^{16}$ G. Lungu,${ }^{17}$ L. Lyons,,${ }^{41}$ J. Lys,${ }^{28}$ R. Lysak, ${ }^{1}$ E. Lytken, ${ }^{46}$ P. Mack,${ }^{25}$ D. MacQueen, ${ }^{32}$ R. Madrak, ${ }^{16}$ K. Maeshima, ${ }^{16}$ P. Maksimovic, ${ }^{24}$ G. Manca, ${ }^{29}$ F. Margaroli, ${ }^{5}$ R. Marginean, ${ }^{16}$ C. Marino, ${ }^{23}$ A. Martin, ${ }^{58}$ M. Martin, ${ }^{24}$ V. Martin, ${ }^{37}$ M. Martínez, ${ }^{3}$ T. Maruyama, ${ }^{53}$ H. Matsunaga, ${ }^{53}$

M. E. Mattson, ${ }^{56}$ R. Mazini, ${ }^{32}$ P. Mazzanti, ${ }^{5}$ K. S. McFarland,${ }^{47}$ D. McGivern,${ }^{30}$ P. McIntyre, ${ }^{51}$ P. McNamara, ${ }^{50}$

R. McNulty, ${ }^{29}$ A. Mehta, ${ }^{29}$ S. Menzemer, ${ }^{31}$ A. Menzione, ${ }^{44}$ P. Merkel,${ }^{46}$ C. Mesropian, ${ }^{48}$ A. Messina, ${ }^{49}$ M. von der Mey, ${ }^{8}$ T. Miao, ${ }^{16}$ N. Miladinovic, ${ }^{6}$ J. Miles, ${ }^{31}$ R. Miller, ${ }^{34}$ J. S. Miller, ${ }^{33}$ C. Mills, ${ }^{10}$ M. Milnik, ${ }^{25}$ R. Miquel, ${ }^{28}$ S. Miscetti, ${ }^{18}$

G. Mitselmakher, ${ }^{17}$ A. Miyamoto, ${ }^{26}$ N. Moggi, ${ }^{5}$ B. Mohr, ${ }^{8}$ R. Moore, ${ }^{16}$ M. Morello, ${ }^{44}$ P. Movilla Fernandez,${ }^{28}$ J. Mülmenstädtt, ${ }^{28}$ A. Mukherjee, ${ }^{16}$ M. Mulhearn, ${ }^{31}$ Th. Muller, ${ }^{25}$ R. Mumford,${ }^{24}$ P. Murat, ${ }^{16}$ J. Nachtman, ${ }^{16}$ S. Nahn, ${ }^{58}$ I. Nakano, ${ }^{39}$ A. Napier, ${ }^{54}$ D. Naumov, ${ }^{36}$ V. Necula,,${ }^{17}$ C. Neu, ${ }^{43}$ M. S. Neubauer, ${ }^{9}$ J. Nielsen, ${ }^{28}$ T. Nigmanov, ${ }^{45}$ 
L. Nodulman, ${ }^{2}$ O. Norniella, ${ }^{3}$ T. Ogawa, ${ }^{55}$ S. H. Oh, ${ }^{15}$ Y. D. Oh, ${ }^{27}$ T. Okusawa, ${ }^{40}$ R. Oldeman, ${ }^{29}$ R. Orava, ${ }^{22}$ K. Osterberg, ${ }^{22}$ C. Pagliarone, ${ }^{44}$ E. Palencia,${ }^{11}$ R. Paoletti, ${ }^{44}$ V. Papadimitriou, ${ }^{16}$ A. Papikonomou, ${ }^{25}$ A. A. Paramonov, ${ }^{13}$ B. Parks ${ }^{38}$ S. Pashapour, ${ }^{32}$ J. Patrick,${ }^{16}$ G. Pauletta, ${ }^{52}$ M. Paulini,${ }^{12}$ C. Paus,${ }^{31}$ D. E. Pellett, ${ }^{7}$ A. Penzo,${ }^{52}$ T. J. Phillips,${ }^{15}$ G. Piacentino, ${ }^{44}$ J. Piedra, ${ }^{11}$ K. Pitts, ${ }^{23}$ C. Plager,${ }^{8}$ L. Pondrom,${ }^{57}$ G. Pope,${ }^{45}$ X. Portell, ${ }^{3}$ O. Poukhov,${ }^{14}$ N. Pounder, ${ }^{41}$ F. Prakoshyn, ${ }^{14}$ A. Pronko, ${ }^{16}$ J. Proudfoot,${ }^{2}$ F. Ptohos, ${ }^{18}$ G. Punzi,${ }^{44}$ J. Pursley, ${ }^{24}$ J. Rademacker, ${ }^{41}$ A. Rahaman, ${ }^{45}$ A. Rakitin, ${ }^{31}$ S. Rappoccio, ${ }^{21}$ F. Ratnikov,${ }^{50}$ B. Reisert, ${ }^{16}$ V. Rekovic, ${ }^{36}$ N. van Remortel, ${ }^{22}$ P. Renton, ${ }^{41}$ M. Rescigno, ${ }^{49}$ S. Richter, ${ }^{25}$ F. Rimondi,${ }^{5}$ K. Rinnert,${ }^{25}$ L. Ristori, ${ }^{44}$ W. J. Robertson, ${ }^{15}$ A. Robson, ${ }^{20}$ T. Rodrigo, ${ }^{11}$ E. Rogers,${ }^{23}$ S. Rolli ${ }^{54}$ R. Roser, ${ }^{16}$ M. Rossi,${ }^{52}$ R. Rossin, ${ }^{17}$ C. Rott, ${ }^{46}$ A. Ruiz,${ }^{11}$ J. Russ, ${ }^{12}$ V. Rusu, ${ }^{13}$ D. Ryan,,${ }^{54}$ H. Saarikko, ${ }^{22}$ S. Sabik, ${ }^{32}$ A. Safonov, ${ }^{7}$ W. K. Sakumoto, ${ }^{47}$ G. Salamanna, ${ }^{49}$ O. Salto, ${ }^{3}$ D. Saltzberg, ${ }^{8}$ C. Sanchez, ${ }^{3}$ L. Santi, ${ }^{52}$ S. Sarkar, ${ }^{49}$ K. Sato, ${ }^{53}$ P. Savard, ${ }^{32}$ A. Savoy-Navarro, ${ }^{16}$ T. Scheidle, ${ }^{25}$ P. Schlabach, ${ }^{16}$ E. E. Schmidt, ${ }^{16}$ M. P. Schmidt, ${ }^{58}$ M. Schmitt, ${ }^{37}$ T. Schwarz, ${ }^{33}$ L. Scodellaro, ${ }^{11}$ A. L. Scott, ${ }^{10}$ A. Scribano, ${ }^{44}$ F. Scuri, ${ }^{44}$ A. Sedov,${ }^{46}$ S. Seidel, ${ }^{36}$ Y. Seiya,${ }^{40}$ A. Semenov, ${ }^{14}$ F. Semeria ${ }^{5}$ L. Sexton-Kennedy, ${ }^{16}$ I. Sfiligoi, ${ }^{18}$ M. D. Shapiro, ${ }^{28}$ T. Shears,${ }^{29}$ P. F. Shepard, ${ }^{45}$ D. Sherman,${ }^{21}$ M. Shimojima, ${ }^{53}$ M. Shochet, ${ }^{13}$ Y. Shon ${ }^{57}$ I. Shreyber,${ }^{35}$ A. Sidoti, ${ }^{44}$ J. Siegrist, ${ }^{28}$ A. Sill, ${ }^{16}$ P. Sinervo, ${ }^{32}$ A. Sisakyan, ${ }^{14}$ J. Sjolin, ${ }^{41}$ A. Skiba, ${ }^{25}$ A. J. Slaughter, ${ }^{16}$ K. Sliwa, ${ }^{54}$ D. Smirnov ${ }^{36}$ J. R. Smith, ${ }^{7}$ F. D. Snider, ${ }^{16}$ R. Snihur, ${ }^{32}$ M. Soderberg, ${ }^{33}$ A. Soha,${ }^{7}$ S. Somalwar, ${ }^{50}$ V. Sorin, ${ }^{34}$ J. Spalding, ${ }^{16}$ F. Spinella, ${ }^{44}$ P. Squillacioti, ${ }^{44}$ M. Stanitzki, ${ }^{58}$ A. Staveris-Polykalas, ${ }^{44}$ R. St. Denis, ${ }^{20}$ B. Stelzer, ${ }^{8}$ O. Stelzer-Chilton, ${ }^{32}$ D. Stentz,${ }^{37}$ J. Strologas, ${ }^{36}$ D. Stuart,${ }^{10}$ J. S. Suh, ${ }^{27}$ A. Sukhanov, ${ }^{17}$ K. Sumorok,${ }^{31}$ H. Sun, ${ }^{54}$ T. Suzuki, ${ }^{53}$ A. Taffard, ${ }^{23}$ R. Tafirout, ${ }^{32}$ R. Takashima, ${ }^{39}$ Y. Takeuchi,${ }^{53} \mathrm{~K}$. Takikawa, ${ }^{53} \mathrm{M}$. Tanaka, ${ }^{2} \mathrm{R}$. Tanaka, ${ }^{39}$ N. Tanimoto, ${ }^{39} \mathrm{M}$. Tecchio, ${ }^{33} \mathrm{P} . \mathrm{K}$. Teng, ${ }^{1} \mathrm{~K}$. Terashi, ${ }^{48}$

S. Tether,${ }^{31}$ J. Thom,${ }^{16}$ A. S. Thompson, ${ }^{20}$ E. Thomson, ${ }^{43}$ P. Tipton,${ }^{47}$ V. Tiwari, ${ }^{12}$ S. Tkaczyk,${ }^{16}$ D. Toback,${ }^{51}$ K. Tollefson, ${ }^{34}$ T. Tomura, ${ }^{53}$ D. Tonelli, ${ }^{44}$ M. Tönnesmann, ${ }^{34}$ S. Torre, ${ }^{44}$ D. Torretta, ${ }^{16}$ S. Tourneur, ${ }^{16} \mathrm{~W}$. Trischuk, ${ }^{32}$ R. Tsuchiya, ${ }^{55}$ S. Tsuno,${ }^{39}$ N. Turini, ${ }^{44}$ F. Ukegawa ${ }^{53}$ T. Unverhau,${ }^{20}$ S. Uozumi,${ }^{53}$ D. Usynin,${ }^{43}$ L. Vacavant,${ }^{28}$ A. Vaiciulis ${ }^{47}$ S. Vallecorsa, ${ }^{19}$ A. Varganov,${ }^{33}$ E. Vataga ${ }^{36}$ G. Velev,${ }^{16}$ G. Veramendi, ${ }^{23}$ V. Veszpremi, ${ }^{46}$ T. Vickey, ${ }^{23}$ R. Vidal, ${ }^{16}$ I. Vila, ${ }^{11}$ R. Vilar, ${ }^{11}$ I. Vollrath, ${ }^{32}$ I. Volobouev, ${ }^{28}$ F. Würthwein, ${ }^{9}$ P. Wagner,${ }^{51}$ R. G. Wagner, ${ }^{2}$ R. L. Wagner, ${ }^{16}$ W. Wagner, ${ }^{25}$ R. Wallny, ${ }^{8}$ T. Walter, ${ }^{25}$ Z. Wan, ${ }^{50}$ M. J. Wang, ${ }^{1}$ S. M. Wang, ${ }^{17}$ A. Warburton, ${ }^{32}$ B. Ward, ${ }^{20}$ S. Waschke, ${ }^{20}$ D. Waters,${ }^{30}$ T. Watts, ${ }^{50} \mathrm{M}$. Weber, ${ }^{28}$ W. C. Wester III, ${ }^{16}$ B. Whitehouse, ${ }^{54}$ D. Whiteson, ${ }^{43}$ A. B. Wicklund, ${ }^{2}$ E. Wicklund, ${ }^{16}$ H. H. Williams, ${ }^{43}$ P. Wilson, ${ }^{16}$ B. L. Winer, ${ }^{38}$ P. Wittich, ${ }^{43}$ S. Wolbers, ${ }^{16}$ C. Wolfe,${ }^{13}$ S. Worm, ${ }^{50}$ T. Wright, ${ }^{33}$ X. Wu, ${ }^{19}$ S. M. Wynne, ${ }^{29}$ S. Xie, ${ }^{32}$ A. Yagil,${ }^{16}$ K. Yamamoto ${ }^{40}$ J. Yamaoka, ${ }^{50}$ T. Yamashita ${ }^{39}$ C. Yang,${ }^{58}$ U. K. Yang, ${ }^{13}$ W. M. Yao, ${ }^{28}$ G. P. Yeh, ${ }^{16}$ J. Yoh, ${ }^{16}$ K. Yorita, ${ }^{13}$ T. Yoshida ${ }^{40}$ I. Yu, ${ }^{27}$ S. S. Yu ${ }^{43}$ J. C. Yun, ${ }^{16}$ L. Zanello, ${ }^{49}$ A. Zanetti, ${ }^{52}$ I. Zaw, ${ }^{21}$ F. Zetti, ${ }^{44}$ X. Zhang, ${ }^{23}$ J. Zhou, ${ }^{50}$ and S. Zucchelli ${ }^{5}$

\title{
(CDF Collaboration)
}

\author{
${ }^{1}$ Institute of Physics, Academia Sinica, Taipei, Taiwan 11529, Republic of China \\ ${ }^{2}$ Argonne National Laboratory, Argonne, Illinois 60439, USA \\ ${ }^{3}$ Institut de Fisica d'Altes Energies, Universitat Autonoma de Barcelona, E-08193, Bellaterra (Barcelona), Spain \\ ${ }^{4}$ Baylor University, Waco, Texas 76798, USA \\ ${ }^{5}$ Istituto Nazionale di Fisica Nucleare, University of Bologna, I-40127 Bologna, Italy \\ ${ }^{6}$ Brandeis University, Waltham, Massachusetts 02254, USA \\ ${ }^{7}$ University of California, Davis, Davis, California 95616, USA \\ ${ }^{8}$ University of California, Los Angeles, Los Angeles, California 90024, USA \\ ${ }^{9}$ University of California, San Diego, La Jolla, California 92093, USA \\ ${ }^{10}$ University of California, Santa Barbara, Santa Barbara, California 93106, USA \\ ${ }^{11}$ Instituto de Fisica de Cantabria, CSIC-University of Cantabria, 39005 Santander, Spain \\ ${ }^{12}$ Carnegie Mellon University, Pittsburgh, Pennsylvania 15213, USA \\ ${ }^{13}$ Enrico Fermi Institute, University of Chicago, Chicago, Illinois 60637, USA \\ ${ }^{14}$ Joint Institute for Nuclear Research, RU-141980 Dubna, Russia \\ ${ }^{15}$ Duke University, Durham, North Carolina 27708, USA \\ ${ }^{16}$ Fermi National Accelerator Laboratory, Batavia, Illinois 60510, USA \\ ${ }^{17}$ University of Florida, Gainesville, Florida 32611, USA \\ ${ }^{18}$ Laboratori Nazionali di Frascati, Istituto Nazionale di Fisica Nucleare, I-00044 Frascati, Italy \\ ${ }^{19}$ University of Geneva, CH-1211 Geneva 4, Switzerland \\ ${ }^{20}$ Glasgow University, Glasgow G12 8QQ, United Kingdom \\ ${ }^{21}$ Harvard University, Cambridge, Massachusetts 02138, USA \\ ${ }^{22}$ Division of High Energy Physics, Department of Physics, University of Helsinki \\ and Helsinki Institute of Physics, FIN-00014, Helsinki, Finland
}




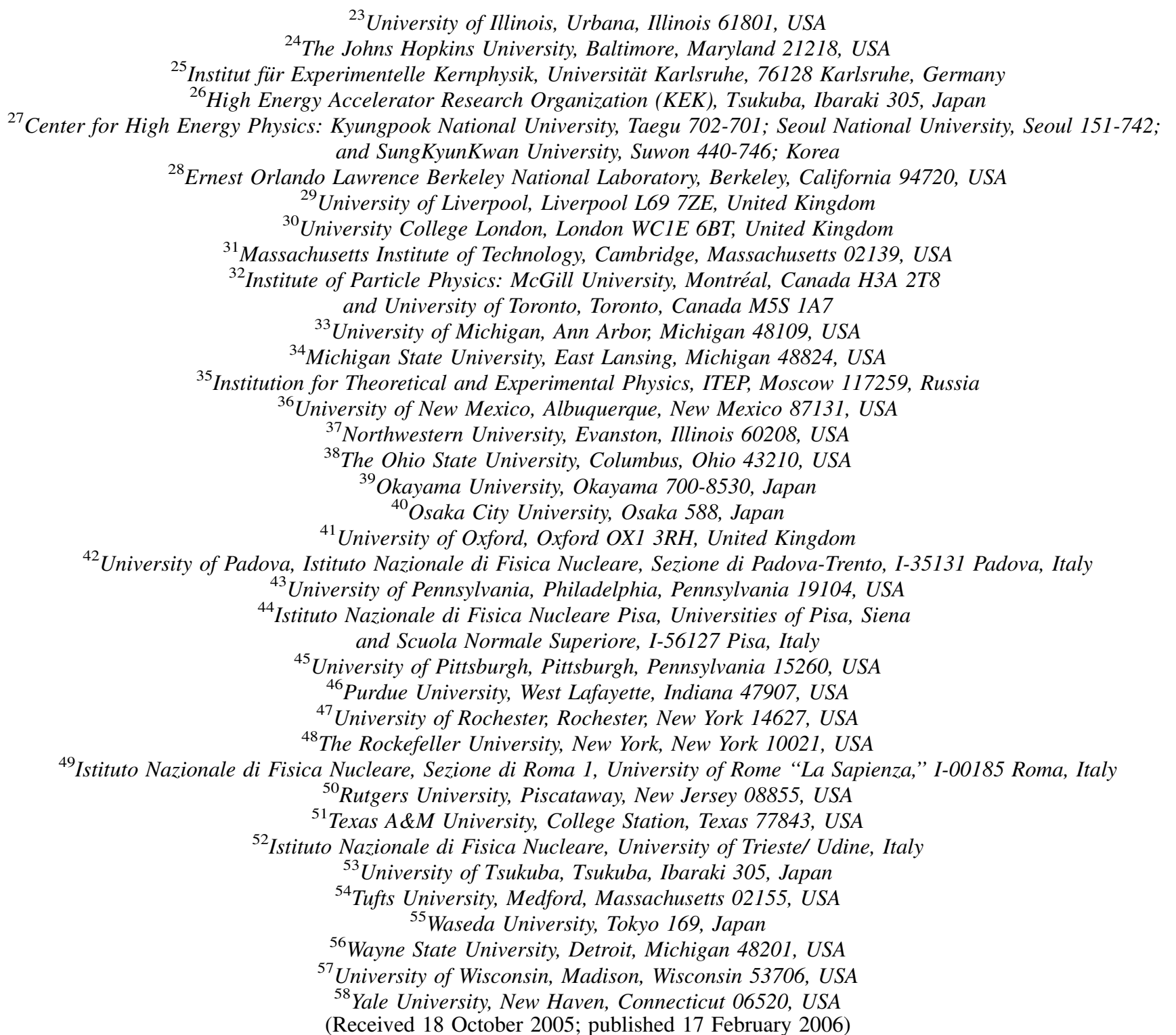

This article presents a measurement of the top quark mass using the CDF II detector at Fermilab. Colliding beams of protons and antiprotons at Fermilab's Tevatron $(\sqrt{s}=1.96 \mathrm{TeV})$ produce top/antitop pairs, which decay to $W^{+} W^{-} b \bar{b}$; events are selected where one $W$ decays to hadrons and the other $W$ decays to either $e$ or $\mu$ plus a neutrino. The data sample corresponds to an integrated luminosity of approximately $318 \mathrm{pb}^{-1}$. A total of $165 t \bar{t}$ events are separated into four subsamples based on jet transverse energy thresholds and the number of $b$ jets identified by reconstructing a displaced vertex. In each event, the reconstructed top quark invariant mass is determined by minimizing a $\chi^{2}$ for the overconstrained kinematic system. At the same time, the mass of the hadronically decaying $W$ boson is measured in the same event sample. The observed $W$ boson mass provides an in situ improvement in the determination of the hadronic jet energy scale. A simultaneous likelihood fit of the reconstructed top quark masses and the $W$ boson invariant masses in the data sample to distributions from simulated signal and background events gives a top quark mass of $173.5_{-3.8}^{+3.9} \mathrm{GeV} / c^{2}$.

DOI: 10.1103/PhysRevD.73.032003

PACS numbers: $12.15 . \mathrm{Ff}, 14.65 . \mathrm{Ha}$

\section{INTRODUCTION}

The top quark is the heaviest observed elementary particle, with a mass roughly 40 times larger than the mass of the $b$ quark. This property of the top quark produces large contributions to electroweak radiative corrections, making more accurate measurements of the top quark mass important for precision tests of the standard model and providing tighter constraints on the mass of the putative Higgs particle. The near-unity Yukawa coupling of the top quark 
also hints at a role for the particle in electroweak symmetry breaking. Improved measurements of the top quark mass are key not only for completing our current description of particle physics, but also for understanding possible physics beyond the standard model.

The top quark was first observed in 1995 during the first run of the Fermilab Tevatron, by CDF [1] and D0 [2]. By the end of run I, the combined measurement of the top quark mass was $178.0 \pm 4.3 \mathrm{GeV} / c^{2}$ [3] using $100-125 \mathrm{pb}^{-1}$ of data per experiment. This article reports a measurement of the top quark mass in the lepton + jets decay channel using the upgraded CDF II detector at Fermilab, with $318 \mathrm{pb}^{-1}$ of $p \bar{p}$ data collected between February 2002 and August 2004. A brief overview of the analysis is as follows.

We scrutinize the data for events where a $t \bar{t}$ pair has been produced and has decayed to two $W$ bosons and two $b$ quarks, where subsequently one $W$ boson decayed to two quarks and the other $W$ boson decayed to an electron or muon and a neutrino. Thus we look for a high-energy electron or muon, momentum imbalance in the detector representing the neutrino, two jets of particles corresponding to the $b$ quarks, and two additional jets corresponding to the hadronic $W$ decay.

Our measurement uses an observable that is strongly correlated with the top quark pole mass, namely, the reconstructed top quark mass. This quantity is determined for each event by minimizing a $\chi^{2}$ function in a kinematic fit to a $t \bar{t}$ final state [4]. In this fit, we apply energy and momentum conservation, constrain both sets of $W$ decay daughters to have the invariant mass of the $W$ boson, and constrain both $W b$ states to have the same mass. The mass reconstruction is complicated by an ambiguity as to which jet represents each quark in the final state. However, since the above procedure yields an overconstrained system, we can choose which jet to assign to each quark based on the fit quality. In addition, some jets are experimentally identified as arising from $b$ quarks by utilizing the relatively long lifetime of the $b$ quark, reducing the number of allowed jetquark assignments.

The method we use to measure the top quark mass is similar in concept to an analysis performed at CDF using data from run I [5]. We compare the distribution of the reconstructed mass from events in the data with the distributions derived from events simulated at various values of the top quark mass. We also simulate events from the expected background processes. Our measured value is the top quark mass for which the simulated events, when combined with the background, best describe the distribution in the data. We improve the power of the method by separating the events into four subsamples that have different background contamination and different sensitivity to the top quark mass.

An important uncertainty in top mass measurements arises from the uncertainty in the jet energy scale, particu- larly for the two jets from $b$ quarks that are direct decay products of the top quarks. To reduce this uncertainty, we have developed a technique exploiting the fact that the daughters of the hadronically decaying $W$ boson should form an invariant mass consistent with the precisely known $W$ boson mass. We constrain the jet energy scale by comparing the distribution of observed dijet invariant mass for candidate $W$ boson daughter jets with simulated distributions assuming various shifts in the jet energy scale with respect to our nominal scale. We show that this improves the jet energy scale information and is largely independent of the top quark mass. Furthermore, since this information applies in large part to $b$ jets as well, it can be used to significantly reduce the uncertainties in the overall top quark mass measurement. A measurement of the top quark mass without this additional information gives consistent results, albeit with larger overall uncertainties.

A brief outline of this article is as follows: In Sec. II, we describe the CDF II detector used for the analysis and our event selection for $t \bar{t}$ candidates in the lepton + jets channel and give background estimates. Section III explains the corrections we make to the jets measured in our detector, as well as the systematics associated with these corrections that dominate top quark mass measurements. Also described in this section is how we reduce these systematics using the $W$ dijet mass. The machinery for reconstructing distributions of top quark masses and dijet masses is explained in Sec. IV, and our method for fitting these distributions is described in Sec. V. Section VI gives the results of fits to the data, as well as cross-checks for our measurement. The remaining systematics are detailed in Sec. VI, and we conclude in Sec. VIII.

\section{DETECTOR, BACKGROUNDS, AND EVENT SELECTION}

This section begins with an explanation of the $t \bar{t}$ event signature along with a summary of the background processes that can mimic it. The relevant parts of the CDF II detector are briefly described, as well as the Monte Carlo generation and simulation procedures. The event selection and the separation into disjoint subsamples are defined next. Finally, the expected number of background events is discussed.

\section{A. Event signature}

In the standard model, the top quark decays with a very short lifetime $\left(\tau \approx 4 \times 10^{-25} \mathrm{~s}\right)$ and with $\sim 100 \%$ branching ratio into a $W$ boson and a $b$ quark. The $t \bar{t}$ event signature is therefore determined by the decay products of the two $W$ bosons, each of which can produce two quarks or a charged lepton and a neutrino. This analysis considers events in the lepton + jets channel, where one $W$ decays to quarks and the other $W$ decays to $e \nu_{e}$ or $\mu \nu_{\mu}$. In the following, "lepton" will refer exclusively to a candidate electron or muon. Thus, events of interest to this 
measurement have an energetic $e$ or $\mu$, a neutrino, and four jets, two of which are $b$ jets. More jets may be present due to hard gluon radiation from an incoming parton (initialstate radiation, ISR) or from a final-state quark (final-state radiation, FSR). Events where a $W$ boson decays to $\tau \nu_{\tau}$ can also enter the event sample when a secondary electron or muon from the tau decay passes the lepton cuts - about $6 \%$ of identified $t \bar{t}$ events have this decay chain.

There are several non- $t \bar{t}$ processes that have similar signatures and enter into the event sample for this analysis. Events where a leptonically decaying $W$ boson is found in association with QCD production of at least four additional jets, sometimes including a $b \bar{b}$ pair, have the same signature and are an irreducible background. Singly produced top quarks, e.g. $q \bar{q} \rightarrow t \bar{b}$, with a leptonic $W$ decay and additional jets produced via QCD radiation, also have the same signature. Additional background events enter the sample when the $t \bar{t}$ signature is faked. For example, a jet can fake an isolated lepton, albeit with small probability, a neutrino can be mistakenly inferred when the missing energy in the event is mismeasured, and a leptonically decaying $Z$ boson can look like a $W$ if one lepton goes undetected.

\section{B. Detector}

The Collider Detector at Fermilab is a general-purpose detector observing $p \bar{p}$ collisions at Fermilab's Tevatron.
The detector geometry is cylindrical, with the $z$ axis pointing along a tangent to the Tevatron ring, in the direction of proton flight in the accelerator. Transverse quantities such as $E_{T}$ and $p_{T}$ are magnitudes of projections into the plane perpendicular to the $z$ axis. The coordinates $x, y, r$, and $\phi$ are defined in this transverse plane, with the $x$ axis pointing outward from the accelerator ring, and the $y$ axis pointing straight up. The angle $\theta$ is the polar angle measured from the proton direction, and $\eta=-\ln \left(\tan \frac{\theta}{2}\right)$ is the pseudorapidity. When $\eta$ is calculated using the reconstructed interaction point, it is referred to as $\eta_{\text {evt }}$. Figure 1 shows an elevation view of the CDF detector. The relevant subdetectors are described briefly below. A more complete description of the CDF II detector is provided elsewhere [6].

The CDF tracking system is the first detector element crossed by a particle leaving the interaction point in the central region. The silicon detectors [7] provide threedimensional position measurements with very good resolution for charged particles close to the interaction region, allowing extrapolation of tracks back to the collision point and reconstruction of secondary, displaced vertices. There are a total of 722432 channels, with a typical strip pitch of 55-65 $\mu \mathrm{m}$ for axial strips, $60-75 \mu \mathrm{m}$ for $1.2^{\circ}$ small-angle stereo strips, and $125-145 \mu \mathrm{m}$ for $90^{\circ}$ stereo strips. The silicon detector is divided into three separate subdetectors. The layer 00 (L00) is a single-sided layer of silicon mounted directly on the beampipe (made of beryllium),

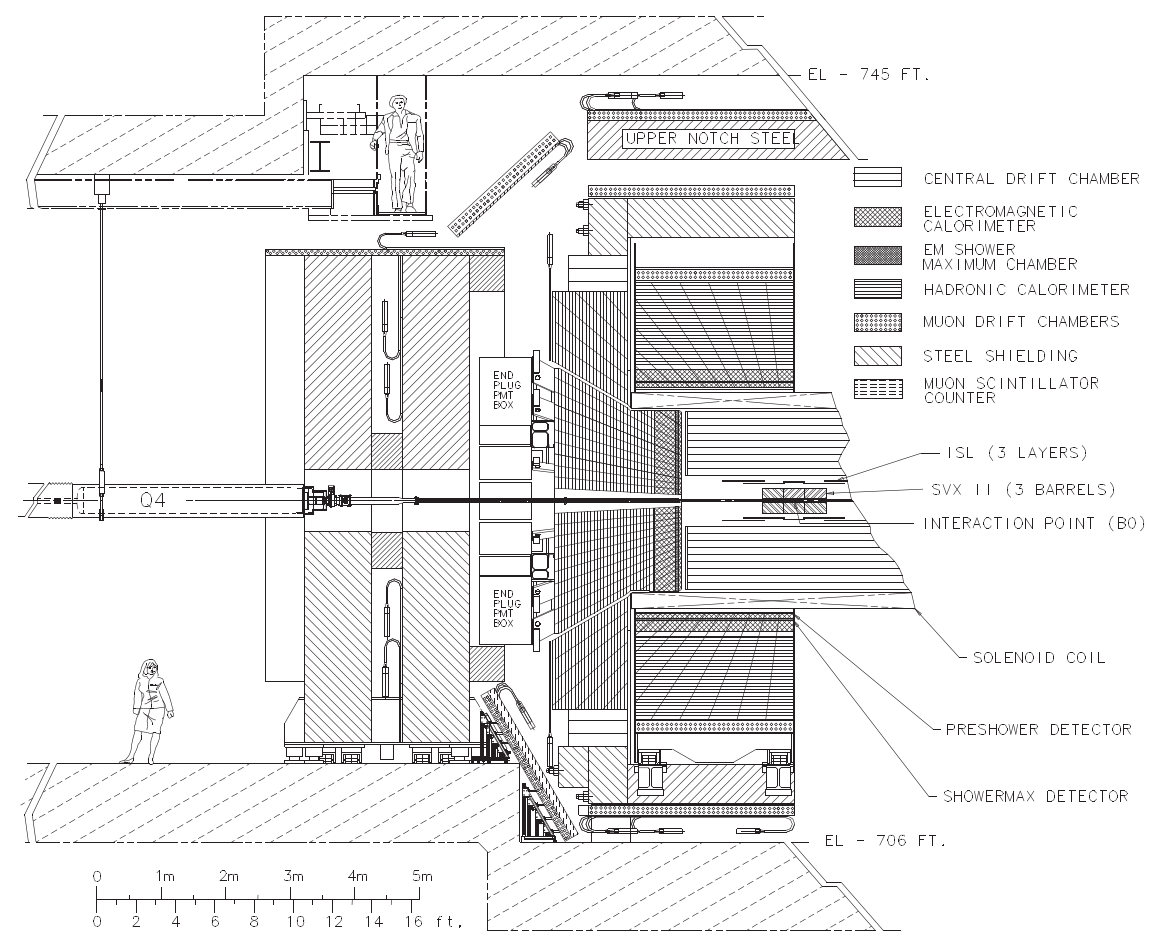

FIG. 1. An elevation view of the CDF II detector. From the collision region outwards, CDF consists of a silicon strip detector, a tracking drift chamber, an electromagnetic calorimeter, a hadronic calorimeter, and muon chambers. 
at a radius of $1.4-1.6 \mathrm{~cm}$, providing an axial measurement close to the collision point. The SVXII detector is $90 \mathrm{~cm}$ long and contains 12 wedges in $\phi$, each with 5 layers of silicon at radii from 2.5 to $10.6 \mathrm{~cm}$. One side of each layer contains strips oriented in the axial direction, and the other side contains $90^{\circ}$ stereo strips in three cases, and $1.2^{\circ}$ small-angle stereo strips in two cases. The intermediate silicon layers (ISL) comprise three additional layers of double-sided silicon at larger radii: at $22 \mathrm{~cm}$ for $|\eta|<1$ and at $20 \mathrm{~cm}$ and $28 \mathrm{~cm}$ for $1<|\eta|<2$. Each layer of the ISL provides axial and small-angle stereo measurements.

The central outer tracker (COT) [8] measures particle locations over a large radial distance, providing precise measurements of track curvature up to about $|\eta|=1$. It is a large open-cell drift chamber with 8 "superlayers" (4 axial and 4 with a $2^{\circ}$ stereo angle), each of which contains 12 wire layers, for a total of 96 layers. There are 30240 wires in total. The COT active volume is $310 \mathrm{~cm}$ in length and covers 43 to $132 \mathrm{~cm}$ in radius. An axial magnetic field of $1.4 \mathrm{~T}$ is provided by a superconducting solenoid surrounding the silicon detectors and central drift chamber.

Particle energies are measured using sampling calorimeters. The calorimeters are segmented into towers with projective geometry. The segmentation of the CDF calorimeters is rather coarse, so that often several particles contribute to the energy measured in one tower.

In the central region, i.e. $|\eta|<1.1$, the calorimeter is divided into wedges subtending $15^{\circ}$ in $\phi$. Each wedge has ten towers, of roughly equal size in $\eta$, on each side of $\eta=$ 0 . The central electromagnetic calorimeter (CEM) [9] contains alternating layers of lead and scintillator, making 18 radiation lengths of material. The transverse energy resolution for high-energy electrons and photons is $\frac{\sigma\left(E_{T}\right)}{E_{T}}=$ $\frac{13.5 \%}{\sqrt{E_{T}[\mathrm{GeV}]}} \oplus 2 \%$. Embedded in the CEM is a shower maximum detector, the CES, which provides good position measurements of electromagnetic showers at a depth of six radiation lengths and is used in electron identification. The CES consists of wire proportional chambers with wires and cathode strips providing stereo position information. The central hadronic calorimeter (CHA) and the end wall hadronic calorimeter (WHA) [10] are of similar construction, with alternating layers of steel and scintillator (4.7 interaction lengths). The WHA fills a gap in the projective geometry between the $\mathrm{CHA}$ and the plug calorimeter.

The calorimetry [11] in the end plugs $(1<|\eta|<3.6)$ has a very complicated tower geometry, but the $15^{\circ}$ wedge pattern is respected. The plug electromagnetic calorimeter (PEM) has lead absorber and scintillating tile read out with wavelength shifting fibers. An electron traversing the PEM passes through 23.2 radiation lengths of material. The energy resolution for high-energy electrons and photons is $\frac{\sigma(E)}{E}=\frac{14.4 \%}{\sqrt{E[\mathrm{GeV}]}} \oplus 0.7 \%$. There is a shower maximum detector (PES), whose scintillating strips measure the po- sition of electron and photon showers. The plug hadronic calorimeter (PHA) has alternating layers of iron and scintillating tile, for a total of 6.8 interaction lengths.

Muon identification is performed by banks of singlewire drift cells four layers deep. The central muon detector (CMU) [12] is located directly behind the hadronic calorimeter in a limited portion of the central region $(|\eta|<$ 0.6). The central muon upgrade (CMP) adds additional coverage in the central region and reduces background with an additional $60 \mathrm{~cm}$ of steel shielding, corresponding to 2.4 interaction lengths at $90^{\circ}$. The central muon extension $(\mathrm{CMX})$ covers the region $0.6<|\eta|<1.0$ and contains eight layers of drift tubes, with the average muon passing through six.

A three-level trigger system is used to select interesting events to be recorded to tape at $\sim 75 \mathrm{~Hz}$ from the bunch crossing rate of $1.7 \mathrm{MHz}$. This analysis uses data from triggers based on high- $p_{T}$ leptons, which come from the leptonically decaying $W$ in the event. The first two trigger levels perform limited reconstruction using dedicated hardware, including the extremely fast tracker (XFT), which reconstructs tracks from the COT in the $r-\phi$ plane with a momentum resolution of better than $2 \% \cdot p_{T}[\mathrm{GeV} / c]$ [13]. The electron trigger requires a coincidence of an XFT track with an electromagnetic cluster in the central calorimeter, while the muon trigger requires that an XFT track points toward a set of hits in the muon chambers. The third level is a software trigger that performs full event reconstruction. Electron and muon triggers at the third level require fully reconstructed objects as in the event selection described below but with looser criteria.

\section{Monte Carlo simulation}

This analysis relies on the use of Monte Carlo (MC) event generation and detector simulation. Event generation is performed by HERWIG v6.505 [14] for $t \bar{t}$ signal samples, and HERWIG, PYTHIA v6.216 [15], and ALPGEN v1.3 [16] for background and control samples.

A detailed description of the CDF detector is used in a simulation that tracks the interactions of particles in each subdetector and fills data banks whose format is the same as the raw data [17]. The GEANT package [18] provides a good description of most interactions, and detailed models are developed and tuned to describe other aspects (for example, the COT ionization and drift properties) so that high-level quantities like tracking efficiency and momentum resolution from the data can be reproduced. The calorimeter simulation is performed using a parameterized shower simulation (GFLASH [19]) tuned to single particle energy response and shower shapes from the data.

\section{Event selection}

A data sample enriched in $t \bar{t}$ events in the lepton + jets channel is selected by looking for events with an electron (muon) with $E_{T}>20 \mathrm{GeV}\left(p_{T}>20 \mathrm{GeV} / c\right)$, missing 
transverse energy $\mathscr{E}_{T}>20 \mathrm{GeV}$, at least three jets with $E_{T}>15 \mathrm{GeV}$, and a fourth jet with $E_{T}>8 \mathrm{GeV}$. This section describes the event selection in detail.

Selected events must contain exactly one well-identified lepton candidate in events recorded by the high- $p_{T}$ lepton triggers. The lepton candidate can be a central electron or a muon observed in the CMU and central muon upgrade detectors or a muon observed in the CMX detector. The trigger efficiencies for leptons in the final sample are high, $\sim 96 \%$ for electrons and $\sim 90 \%$ for muons, and show negligible $p_{T}$ dependence.

Electrons are identified by a high-momentum track in the tracking detectors matched with an energy cluster in the electromagnetic calorimeter with $E_{T}>20 \mathrm{GeV}$. The rate of photons and hadronic matter faking electrons is reduced by requiring the ratio of calorimeter energy to track momentum to be no greater than 2 (unless $p_{T}>50 \mathrm{GeV} / c$, in which case this requirement is not imposed) and by requiring the ratio of hadronic to electromagnetic energy in the calorimeter towers to be less than $0.055+0.00045 \cdot E_{E M}$. Isolated electrons from $W$ decays are preferentially selected over electrons from $b$ or $c$ quark semileptonic decays by requiring the additional calorimeter energy in a cone of $\Delta R=\sqrt{\Delta \phi^{2}+\Delta \eta_{\mathrm{evt}}^{2}}=0.4$ around the cluster to be less than $10 \%$ of the cluster energy. Electrons are rejected if they come from photon conversions to $e^{+} e^{-}$ pairs that have been explicitly reconstructed.

Muons are identified by a high-momentum track in the tracking detectors $\left(p_{T}>20 \mathrm{GeV} / c\right)$ matched with a set of hits in the muon chambers. The calorimeter towers to which the track points must contain energy consistent with a minimum ionizing particle. An isolation cut is imposed, requiring the total calorimeter energy in a cone of $\Delta R=0.4$ around the muon track (excluding the towers through which the muon passed) to be less than $10 \%$ of the track momentum. Cosmic ray muons explicitly identified are rejected. A complete description of electron and muon selection, including all additional cuts used, can be found elsewhere [20].

A neutrino from the leptonic $W$ boson decay is inferred when the observed momentum in the transverse plane does not balance. The missing transverse energy, $\not{E}_{T}$, is formed by projecting each tower energy in the central, wall, and plug calorimeters into the plane transverse to the beams and summing: $\mathbb{E}_{T}=-\left\|\sum_{i} E_{T}^{i} \mathbf{n}_{\mathbf{i}}\right\|$, where $\mathbf{n}_{\mathbf{i}}$ is the unit vector in the transverse plane that points to the $i$ th calorimeter tower. The $\mathscr{E}_{T}$ is corrected using the muon track momentum when a muon is identified in the event. For clusters of towers that have been identified as jets, we apply an additional correction to the $\mathbb{E}_{T}$ due to variation in detector responses relative to the fiducial central region and due to the effects of multiple $p \bar{p}$ interactions. We require the $\mathbb{E}_{T}$ to be at least $20 \mathrm{GeV}$.

Jets are identified by looking for clusters of energy in the calorimeter using a cone algorithm, JETCLU, where the cone radius is $\Delta R=0.4$. Towers with $E_{T}>1 \mathrm{GeV}$ are used as a seed for the jet search, then nearby towers are added to the clusters, out to the maximum radius of 0.4. A final step of splitting and merging is performed such that a tower does not contribute to more than one jet. More details about the jet clustering are available elsewhere [21]. Jet energies are corrected for relative detector response and for multiple interactions, as described in Sec. III A.

Jets can be identified as $b$ jets using a displaced vertex tagging algorithm, which proceeds as follows. The primary event vertex is identified using a fit to all prompt tracks in the event and a beamline constraint. The beamline is defined as a linear fit to the collection of primary vertices for particular running periods. The luminous region described by the beamline has a width of approximately $30 \mu \mathrm{m}$ in the transverse view and $29 \mathrm{~cm}$ in the $z$ direction. Jets with $E_{T}>15 \mathrm{GeV}$ are checked for good quality tracks with both COT and silicon information. When a secondary vertex can be reconstructed from at least two of those tracks, the signed distance between the primary and secondary vertices along the jet direction in the plane transverse to the beams $\left(L_{2 D}\right)$ is calculated, along with its uncertainty $\left[\sigma\left(L_{2 D}\right)\right]$. If $L_{2 D} / \sigma\left(L_{2 D}\right)>7.5$, the jet is considered tagged. The per jet efficiency for $b$ jets in the central region is shown as a function of jet $E_{T}$ in Fig. 2; the algorithm has an efficiency of about $60 \%$ for tagging at least one $b$ jet in a $t \bar{t}$ event. More information concerning $b$ tagging is available elsewhere [22].

An additional $b$ tagging algorithm is used only in a cross-check of this analysis, described in Sec. VIC. The jet probability (JPB) tagger $[23,24]$ calculates the probability of observing the $r-\phi$ impact parameters of the tracks in the jet with respect to the primary interaction vertex, under the hypothesis that the jet does not arise from a heavy flavor quark. In the check described later, a jet is identified as a $b$ jet if it has a JPB value less than $5 \%$. Since

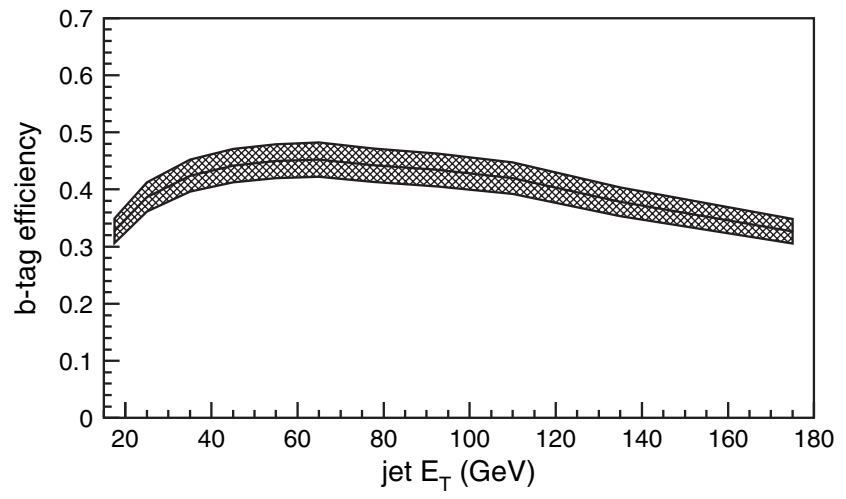

FIG. 2. The efficiency of the secondary vertex $b$-tagging algorithm is shown as a function of jet $E_{T}$ for $b$ jets in the central region of the detector $(|\eta|<1)$, where the tracking efficiency is high. The shaded band gives the $\pm 1 \sigma$ range for $b$-tagging efficiency. The curve is measured using a combination of data and Monte Carlo simulated samples. 
it uses much of the same information, the JPB tag efficiency is correlated with the displaced vertex tag efficiency.

We require at least four jets in the event with $|\eta|<2.0$ in order to reconstruct the $t \bar{t}$ system. In events with more than 4 jets, only the 4 jets with highest $E_{T}$ (the leading 4 jets) are used in jet-quark assignments. The events are separated into four subsamples based on the jet activity. These four categories of events are found to have different background content and different shapes in the reconstruction of the top quark mass for signal events. By treating the subsamples separately, the statistical power of the method is improved. Double-tagged (2-tag) events have two $b$-tagged jets in the event. These events have low background contamination, as well as excellent mass resolution, since the number of allowed jet-quark assignments is small. In this category, we require three jets with $E_{T}>$ $15 \mathrm{GeV}$ and the fourth jet with $E_{T}>8 \mathrm{GeV}$. Tight singletagged [1-tag(T)] events have exactly one $b$-tagged jet in the event, and all four jets with $E_{T}>15 \mathrm{GeV}$. Loose single-tagged [1-tag $(\mathrm{L})]$ events also have exactly one $b$ tag, but the fourth jet has $8 \mathrm{GeV}<E_{T}<15 \mathrm{GeV}$. These two categories have good mass resolution, but 1-tag(L) events have a higher background content than 1-tag(T) events. Finally, 0 -tag events have no $b$ tags and thus a high background contamination. To increase the signal to background ratio (S:B), a tighter $E_{T}$ cut is required: all four jets must have $E_{T}>21 \mathrm{GeV}$.

We find $165 t \bar{t}$ candidates in $318 \mathrm{pb}^{-1}$ of data selected for good quality in all relevant subdetectors. The jet selection requirements for each of the four event types are summarized in Table I, which also lists the expected signal to background ratio and the number of each event type found in the data. The expected S:B assumes a standard model top quark with a mass of $178 \mathrm{GeV} / c^{2}$ (the run I world average) and a corresponding $t \bar{t}$ theoretical cross section of $6.1 \mathrm{pb}$. Since in the 0-tag category we do not

TABLE I. The selection requirements for the four types of events are given. The subsamples have different background content and reconstructed mass shapes. The jet $E_{T}$ requirements apply to the leading four jets in the event, but additional jets are permitted. Also shown are the number of events observed in $318 \mathrm{pb}^{-1}$ of data, and, for purposes of illustration, the expected signal to background ratio $(\mathrm{S}: \mathrm{B})$ assuming a $t \bar{t}$ cross section of $6.1 \mathrm{pb}$. The 0-tag sample category has no independent background estimate.

\begin{tabular}{lccccc}
\hline \hline Category & & 2-tag & 1-tag(T) & 1-tag(L) & 0-tag \\
\hline Jet $E_{T}$ & $\mathrm{j} 1-\mathrm{j} 3$ & $E_{T}>15$ & $E_{T}>15$ & $E_{T}>15$ & $E_{T}>21$ \\
cuts $(\mathrm{GeV})$ & $\mathrm{j} 4$ & $E_{T}>8$ & $E_{T}>15$ & $15>E_{T}>8$ & $E_{T}>21$ \\
\hline$b$-tagged Jets & 2 & 1 & 1 & 0 \\
\hline Expected S:B & $10.6: 1$ & $3.7: 1$ & $1.1: 1$ & N/A \\
\hline Number of events & 25 & 63 & 33 & 44 \\
\hline \hline
\end{tabular}

have an independent background estimate, no estimate of $\mathrm{S}: \mathrm{B}$ is given; about $22 t \bar{t}$ events are expected.

\section{E. Background estimation}

Wherever possible, we obtain an estimate of the background contamination in each subsample that is nearly independent of the observed number of events in that subsample; adding this information as a constraint in the likelihood fit a priori improves the result.

The amount and composition of the background contamination depends strongly on the number of jets with $b$ tags. In the double $b$-tagged sample, the background contribution is very small. In the single $b$-tagged sample, the dominant backgrounds are $W+$ multijet events and non- $W$ QCD events where the primary lepton is not from a $W$ decay. The $W+$ multijet events contain either a heavy flavor jet or a light flavor jet mistagged as a heavy flavor jet. In the events with no $b$ tag, $W+$ multijet production dominates, and the jets are primarily light flavor since there are no $b$ tags.

Table II gives estimates for the background composition in each tagged subsample. Note that some of the estimates in Table II for the various background processes are correlated, so the uncertainty on the total background is not simply the sum in quadrature of the component uncertainties. The procedures for estimating each background type are described in the following sections and are detailed elsewhere [22].

\section{Non-W (QCD) background}

For the non- $W$ background (QCD multijet events), a data-driven technique estimates the contribution to the signal sample. The sideband regions of the lepton isolation $(>0.2)$ vs $\mathbb{E}_{T}(<15 \mathrm{GeV})$ plane (after subtracting the expected $t \bar{t}$ and $W+$ multijet contributions) are used to predict the number of QCD multijet events in the signal region, assuming no correlation between the isolation and $\mathbb{E}_{T}$.

\section{2. $W+$ multijet backgrounds}

Simulated samples of $W+$ multijet backgrounds are obtained using the ALPGEN generator, which produces

TABLE II. The sources and expected numbers of background events in the three subsamples with $b$ tags.

\begin{tabular}{lccc}
\hline \hline \multirow{2}{*}{ Source } & \multicolumn{3}{c}{ Expected Background } \\
\cline { 2 - 4 } & 2-tag & 1-tag(T) & 1-tag $(\mathrm{L})$ \\
\hline Non- $W(\mathrm{QCD})$ & $0.31 \pm 0.08$ & $2.32 \pm 0.50$ & $2.04 \pm 0.54$ \\
$W b \bar{b}+W c \bar{c}+W c$ & $1.12 \pm 0.43$ & $3.91 \pm 1.23$ & $6.81 \pm 1.85$ \\
$W+$ light jets & $0.40 \pm 0.08$ & $3.22 \pm 0.41$ & $4.14 \pm 0.53$ \\
$W W / W Z$ & $0.05 \pm 0.01$ & $0.45 \pm 0.10$ & $0.71 \pm 0.13$ \\
Single top & $0.008 \pm 0.002$ & $0.49 \pm 0.09$ & $0.60 \pm 0.11$ \\
\hline Total & $1.89 \pm 0.52$ & $10.4 \pm 1.72$ & $14.3 \pm 2.45$ \\
\hline \hline
\end{tabular}


multiple partons associated with a $W$ boson using an exact leading order matrix element calculation. The generator is interfaced with HERWIG to simulate parton showering and hadronization. ALPGEN describes the kinematics of events with high jet multiplicity very well, but it suffers from a large theoretical uncertainty in the normalization due to the choice of $Q^{2}$ scale and next-to-leading order (NLO) effects. Thus, the normalization for these backgrounds is taken from the data. The normalization for the $W+$ multijet background in the subsamples requiring $b$ tags comes from the $W+$ multijet events before tagging, after subtracting the expected contributions for $t \bar{t}$ and non- $W$ processes. Because of this procedure, the tagged background predictions are weakly coupled to the observed numbers of events in the tagged subsamples. Using the same procedure, the 0 -tag background estimate would be strongly coupled to the number of observed 0 -tag events. In order to avoid this correlation in the likelihood fit, no background constraint is used for the 0-tag sample.

The major contributions for the $W+$ heavy flavor backgrounds, i.e. events with a $b$ tag on a real $b$ or $c$ jet, come from the $W b \bar{b}, W c \bar{c}$, and $W c$ processes. The fractions of inclusive $W+$ multijet events that contain $b \bar{b}$ pairs, $c \bar{c}$ pairs, and single $c$ quarks are estimated using the ALPGEN/HERWIG Monte Carlo samples after a calibration to the parallel fractions in inclusive jet data. Then the contribution of each background type to the data sample is determined by multiplying the corresponding fraction, the event tagging efficiency for the particular configuration of $b$ and $c$ jets, and the number of $W+$ multijet events in the data before $b$ tagging.

Another $W+$ multijet contribution comes from events where a light flavor jet is misidentified as a heavy flavor jet. Using jet data events, a per jet mistag rate is determined as a function of the number of tracks, $E_{T}, \eta$, and $\phi$ of the jet, and the scalar sum of $E_{T}$ for all jets with $E_{T}>10 \mathrm{GeV}$ and $|\eta|<2$.4. The mistag rate is then applied to pretag data events in the signal region to obtain the $W+$ light flavor contribution.

\section{Other backgrounds}

There are other minor contributions to the backgrounds: diboson production ( $W W, W Z$, and $Z Z$ ) associated with jets, and single top production. We use ALPGEN Monte Carlo samples to estimate their acceptance. The NLO cross section values $[25,26]$ are used for normalization.

\section{JET CORRECTIONS AND SYSTEMATICS}

Jets of particles arising from quarks and gluons are the most important reconstructed objects in the top quark mass measurement but are measured with poor energy resolution. The jet measurements therefore make the largest contribution to the resolution of the mass reconstruction de- scribed in Sec. IV. Additionally, systematic uncertainties on the jet energy measurements are the dominant source of systematic uncertainty on the top quark mass. We describe here the corrections applied to the measured jet energies, as well as the systematic uncertainties on our modeling of the jet production and detector response. A more thorough treatment of these topics is available elsewhere [27]. Finally, we introduce the jet energy scale quantity $\Delta_{\mathrm{JES}}$, which is measured in situ using the $W$ boson mass resonance.

\section{A. Jet corrections}

Matching reconstructed jets to quarks from the $t \bar{t}$ decay has both theoretical and experimental complications. A correspondence generally can be assumed between measured jet quantities and the kinematics of partons from the hard interaction and decay. A series of corrections are made to jet energies in order to best approximate the corresponding quark energies. Measured jet energies have a poor resolution and are treated as uncertain quantities in the mass reconstruction. The measured angles of the jets, in contrast, are good approximations of the corresponding quark angles, so they are used without corrections and are fixed in the mass reconstruction.

\section{Tower calibrations}

Before clustering into jets, the calorimeter tower energies are calibrated as follows. The overall electromagnetic scale is set using the peak of the dielectron mass resonance resulting from decays of the $Z$ boson. The scale of the hadronic calorimeters is set using test beam data, with changes over time monitored using radioactive sources and the energy deposition of muons from $J / \psi$ decays, which are minimum ionizing particles in the calorimeter. Tower-to-tower uniformity for the CEM is achieved by requiring the ratio of electromagnetic energy to track momentum $(E / p)$ of electrons to be the same across the calorimeter. In the CHA and WHA, the $J / \psi \rightarrow \mu \mu$ minimum ionizing particles are also used to equalize the response of towers. For the PEM and PHA, where tracks are not available, the tower-to-tower calibrations use a laser calibration system and ${ }^{60} \mathrm{Co}$ sourcing. The WHA calorimeter also has a sourcing system to monitor changes in the tower gains.

\section{Process-independent corrections}

After clustering, jets are first corrected with a set of "generic" jet corrections, so called because they are intended to be independent of the particular process under consideration. For these corrections, the quark $p_{T}$ distribution is assumed to be flat. Since some of the corrections are a function of jet $p_{T}$, and since the jet resolution is nonnegligible, this assumption has a considerable effect on the derived correction. 
These generic jet corrections scale the measured jet four-vector to account for a set of well-studied effects. First, a dijet balancing procedure is used to determine and correct for variations in the calorimeter response to jets as a function of $\eta$. These variations are due to different detector technology, to differing amounts of material in the tracking volume and the calorimeters, and to uninstrumented regions. In dijet balancing, events are selected with two and only two jets, one in the well-understood central region $(0.2<|\eta|<0.6)$. A correction is determined such that the transverse momentum of the other jet, called the probe jet, as a function of its $\eta$, is equal on average to that of the central jet. This relative correction ranges from about $+15 \%$ to $-10 \%$ and can be seen in Fig. 4 in Sec. III B.

After a small correction for the extra energy deposited by multiple collisions in the same accelerator bunch crossing, a correction for calorimeter nonlinearity is applied so that the jet energies correspond to the most probable incone hadronic energy assuming a flat $p_{T}$ distribution. First, the response of the calorimeter to hadrons is measured using $E / p$ of single tracks in the data. Studies of energy flow and jet shapes in the data also constrain the modeling of jet fragmentation. After tuning the simulation to model what we observe in the data, the correction $(+10 \%$ to $+30 \%$, depending on jet $p_{T}$ ) is determined using a simulated sample of dijet events covering a large $p_{T}$ range.
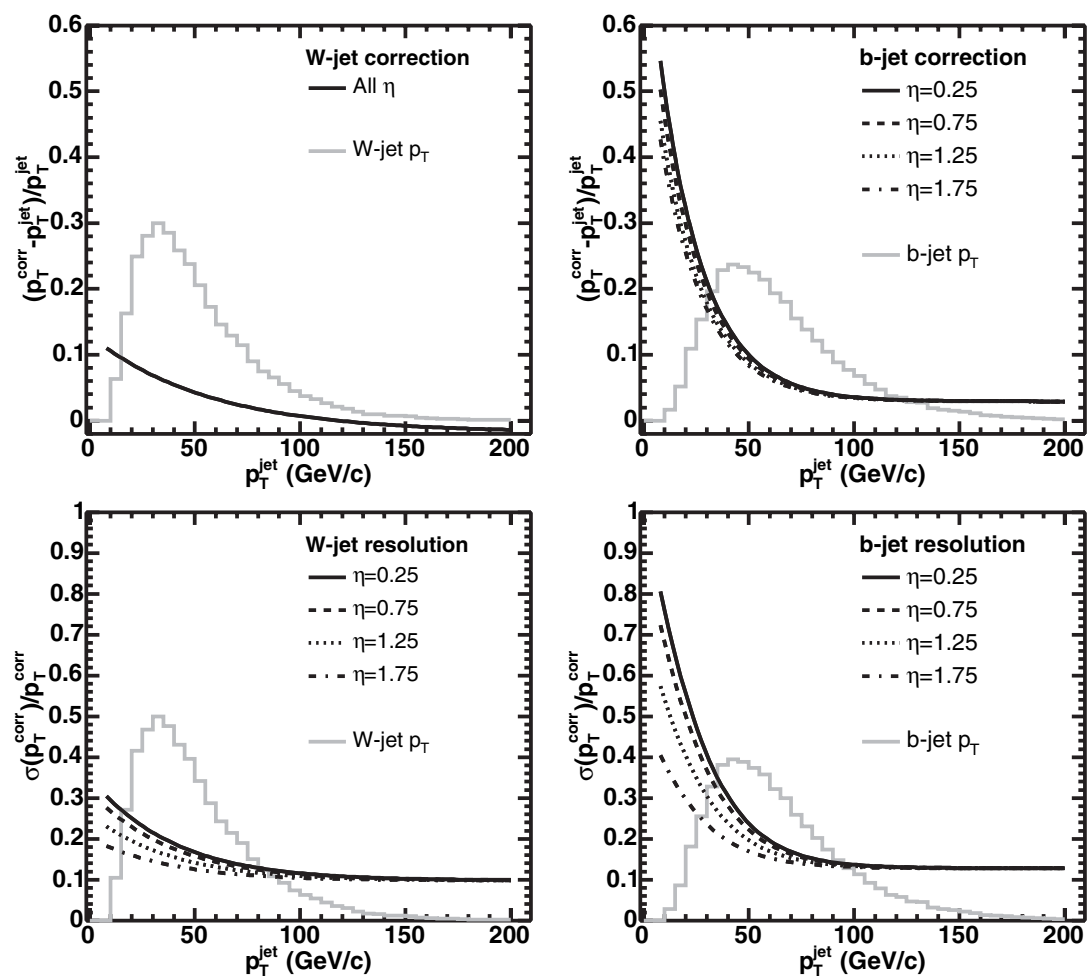

FIG. 3. The $t \bar{t}$-specific corrections are shown for $W$ jets (left) and $b$ jets (right) as a function of jet $p_{T}$ for several values of $|\eta|$. On the top is the correction factor, and on the bottom is the fractional resolution passed to the fitter. The histograms give the distributions of jet $p_{T}$ (arbitrarily normalized) from a signal Monte Carlo sample with generated top quark mass of $178 \mathrm{GeV} / \mathrm{c}^{2}$. 
their correction depends only on $p_{T}^{\text {jet }}$. The MPV is chosen, rather than the mean of the asymmetric distribution, in order to accurately correct as many jets as possible in the core of the distribution. This increases the number of events for which the correct jet-quark assignment is chosen by the fitter (see below), resulting in a narrower core for the reconstructed mass distribution. A corresponding resolution is found by taking the symmetric window about the MPV of the jet response that includes $68 \%$ of the total area. Figure 3 shows the corrections and resolutions as a function of jet $p_{T}$ for several values of $|\eta|$.

As a final step in correcting the jet four-vector, the jet momentum is held fixed while the jet energy is adjusted so that the jet has a mass according to its flavor hypothesis. A mass of $0.5 \mathrm{GeV} / c^{2}$ is used for $W$ jets, and a mass of $5.0 \mathrm{GeV} / c^{2}$ is used for $b$ jets. This is done to match the generator-level quarks used to derive the $t \bar{t}$-specific corrections.

\section{B. Systematic uncertainties on jet energy scale}

There are significant uncertainties on many aspects of the measurement of jet energies. Some of these are in the form of uncertainties on the energy measurements themselves; some are uncertainties on the detector simulation, which is used to derive many corrections and ultimately to extract the top quark mass; still others are best understood as theoretical uncertainties on jet production and fragmentation models used in the generators.

\section{Calorimeter response relative to central}

The systematic uncertainties in the calorimeter response is relative to the central calorimeter range from $0.5 \%$ to $2.5 \%$ for jets used in this analysis. The uncertainties account for the residual $\eta$ dependence after dijet balancing,

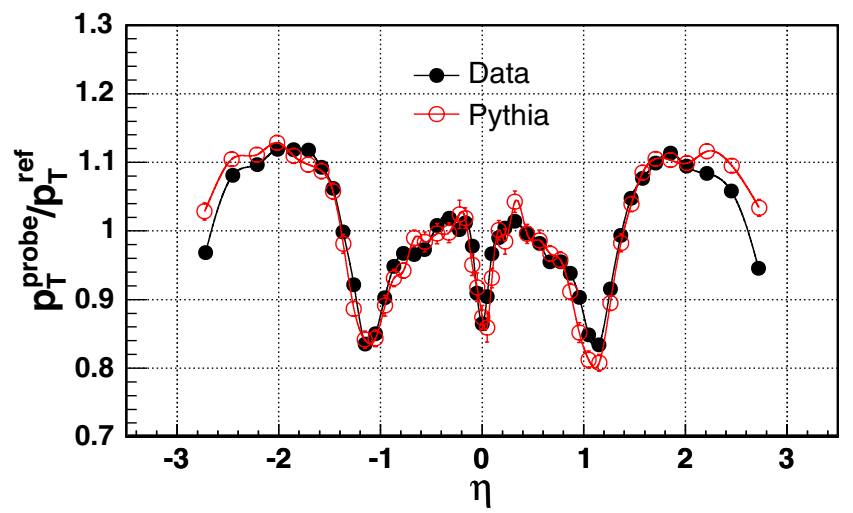

FIG. 4 (color online). Results of the dijet balancing procedure are shown for data and simulated dijet events with $p_{T}^{\text {jet }}>$ $20 \mathrm{GeV} / c$. Probe jets from throughout the detector are compared with a reference jet in the central region; the ratio of the $p_{T}$ of the jets is plotted as a function of the probe jet $\eta$. The simulation models well the detector response as a function of $\eta$. biases in the dijet balancing procedure (especially near the uninstrumented regions), and the variation of the plug calorimeter response with time. Photon-jet balancing is used to check the $\eta$ dependence after corrections in data and simulated events, and the residual differences in this comparison are also included in the systematic uncertainty. Figure 4 shows the dijet balancing as a function of the probe jet pseudorapidity, demonstrating that the simulation models well the detector response for $|\eta|<2$.0. Since differing response in neighboring regions of the detector is the primary source of biased jet angle measurements, the plot also demonstrates that we can expect angle biases to be well modeled in the simulated events.

\section{Modeling of hadron jets}

The main systematic uncertainties at the hadronic level are obtained by propagating the uncertainties on the single particle response and the fragmentation, which are determined from studies on the data. Smaller contributions are included from the comparison of data and Monte Carlo simulation of the calorimeter response close to tower boundaries in azimuth and from the stability of the calorimeter calibration with time. There is also a small uncertainty on the energy deposited by additional $p \bar{p}$ interactions. In all, this uncertainty varies from $1.5 \%$ to $3.0 \%$, depending on jet $p_{T}$, and only accounts for variations that affect the energy inside the jet cone.

\section{Modeling of out-of-cone energy}

The uncertainty on the fraction of energy contained in the jet cone (also primarily due to jet fragmentation modeling) is estimated in two parts - one between $R=0.4$ and $R=1.3$ and the other for $R>1.3$. This systematic uncertainty, which is roughly $9 \%$ at very low jet $p_{T}$ but falls rapidly to $<2 \%$ for $p_{T}>70 \mathrm{GeV} / c$, is determined by comparing the energy flow in jets from data and Monte Carlo for various event topologies.

\section{Modeling of underlying event}

The underlying event deposits energy uniformly in calorimeter towers throughout the detector, some of which are clustered into jets. Such energy is subtracted from the jet energy in the corrections. The uncertainty on this correction decreases rapidly from $2 \%$ at very low $p_{T}$ to less than $0.5 \%$ at about $35 \mathrm{GeV} / c$.

\section{Total uncertainty}

The systematic uncertainties on jet energies for jets in the reference central region $(0.2<|\eta|<0.6)$ are shown as a function of $p_{T}$ in Fig. 5. For other $\eta$ regions, only the contribution of the "relative response" uncertainty changes. The black line gives the total uncertainty on the jet energy measurement, obtained by adding in quadrature the contributions described above. 
Events in which a jet recoils against a high-energy photon are used to check the absolute corrections. We compare the corrected jet energy to the photon energy, which is well calibrated using $Z \rightarrow e^{+} e^{-}$decays. This $\gamma$-jet balancing is performed on data and Monte Carlo samples, as a function of photon $E_{T}$ and jet $\eta$, as a crosscheck of the energy corrections and systematic uncertainties described above. Figure 6 shows a comparison of the $\gamma$-jet balancing in data and Monte Carlo after all jet corrections, along with the $\pm 1 \sigma$ range of the jet energy systematics. The agreement provides confidence that the systematic uncertainties are reasonable.

The systematic uncertainties on jet energies described here are understood to apply to all jets. Clearly additional flavor-specific or process-specific uncertainties could be present. In particular, any systematics specific to the $b$ jets are extremely important in a measurement of the top quark mass and could arise from mismodeling of $b$ quark fragmentation, semileptonic decays, or color connections not present in the $W$ boson decay system. Uncertainties from these sources have been studied and found to be relatively small; see Sec. VII A.

\section{Jet energy scale}

Since the jet energy systematics described in the previous section generate the dominant systematic uncertainty on the top quark mass measurement, a method has been developed to further constrain those systematics using the $W$ boson mass resonance in situ. In particular, we measure

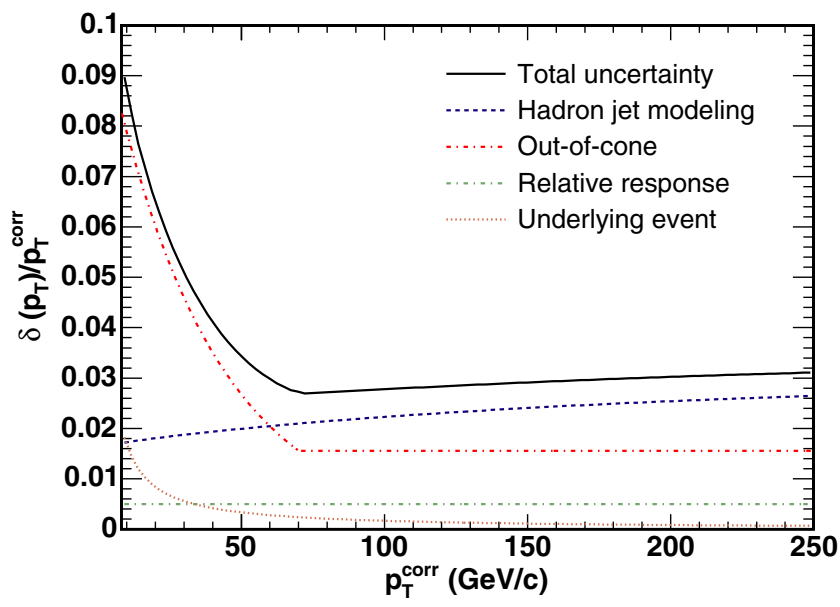

FIG. 5 (color online). The systematic uncertainties on jet energy are shown for jets in the central calorimeter $(0.2<|\eta|<$ 0.6 ). For noncentral jets, the total uncertainty has a different contribution from the eta-dependent uncertainty. In this plot the corrected jet transverse momentum $p_{T}^{\text {corr }}$ is the processindependent estimate of the parton $p_{T}$. At low $p_{T}^{\text {corr }}$, the main contribution to the systematic is from the uncertainty on the fraction of jet energy lost outside the cone, while at high $p_{T}^{\text {corr }}$ it is from the linearity corrections to obtain an absolute jet energy scale. a parameter $\Delta_{\mathrm{JES}}$ that represents a shift in the jet energy scale from our default calibration.

Rather than defining $\Delta_{\mathrm{JES}}$ as a constant percentage shift of the jet energies, we define it in units of the total nominal jet energy scale uncertainty $\left(\sigma_{c}\right)$, which is derived from the extrinsic calibration procedures above. This $\sigma_{c}$ is the quantity depicted in Fig. 5 for central jets. Thus $\Delta_{\mathrm{JES}}=$ $0 \sigma_{c}$ corresponds to our default jet energy scale; $\Delta_{\mathrm{JES}}=$ $1 \sigma_{c}$ implies a shift in all jet energies by 1 standard deviation in the uncertainty defined above; and so on. This choice has two consequences. The first is that the effect of a shift in $\Delta_{\mathrm{JES}}$ is different for jets with different $p_{T}$ and $\eta$. For example, jets with very low $p_{T}$ have a larger fractional uncertainty and therefore have a larger fractional shift with a $1 \sigma_{c}$ change in $\Delta_{\mathrm{JES}}$. The second is that it is easy to incorporate the independent estimate of the jet energy systematics (with its $p_{T}$ and $\eta$ dependence) by constraining $\Delta_{\mathrm{JES}}$ using a Gaussian centered at $0 \sigma_{c}$ with a width of $1 \sigma_{c}$.

As described in Sec. III B, the jet energy scale uncertainty comprises many small effects, which have different

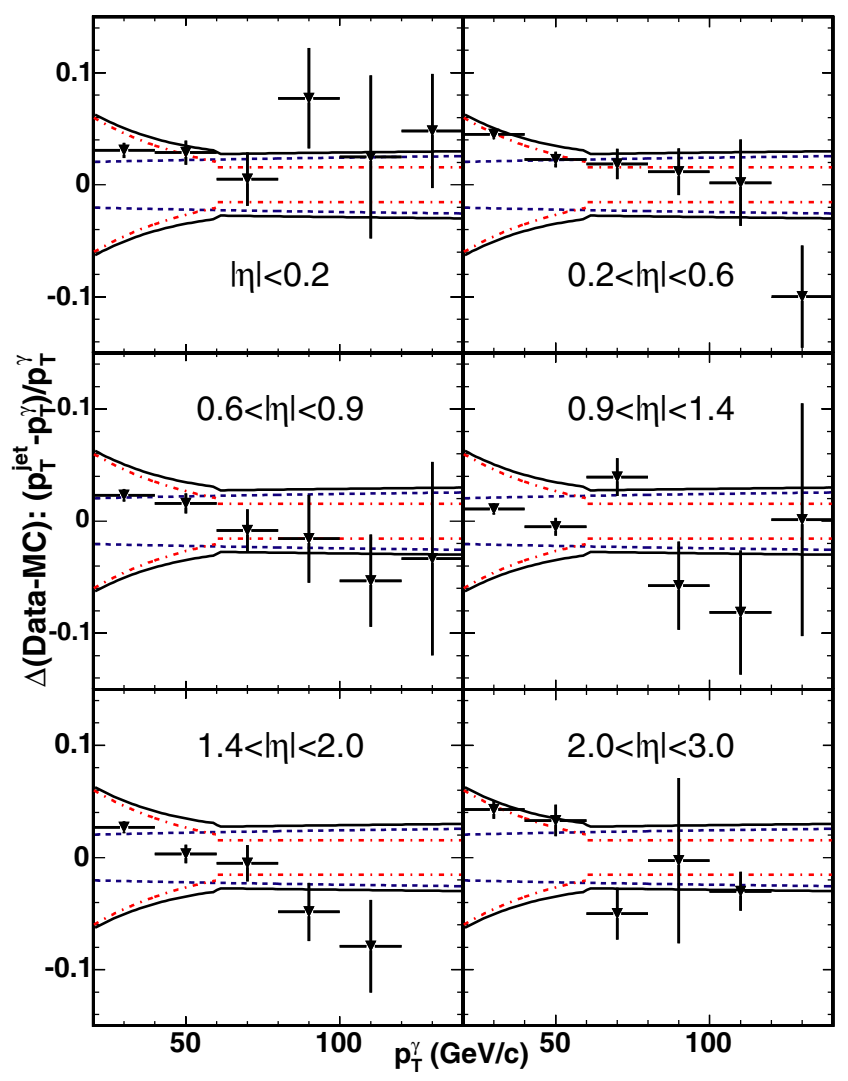

FIG. 6 (color online). For $\gamma$-jet events in both data and simulation, we find the fractional difference in $p_{T}$ between the jet and the photon after all jet corrections are applied. Plotted here, for different ranges of jet $\eta$, is the difference between this quantity in data and simulated events as a function of photon $p_{T}$. The solid lines show the $\pm 1 \sigma$ range given by the jet energy systematics. The other lines follow the same definitions as in Fig. 5. 
dependences on jet $\eta$ and $p_{T}$. With more statistics, we would choose to measure the various effects independently. Currently, however, we make the approximation of assuming that a single value of the parameter $\Delta_{\mathrm{JES}}$ applies to all jets in the sample; that is, we measure a value of $\Delta_{\mathrm{JES}}$ that is averaged over jets in the sample. Additionally, by construction, our $\Delta_{\mathrm{JES}}$ measurement is primarily sensitive to jets from the hadronic $W$ decay. We estimate the effect of this approximation as a systematic uncertainty on the top quark mass measurement.

\section{MASS RECONSTRUCTION}

In this section, we describe the procedures for determining in each event the reconstructed top quark mass $m_{t}^{\text {reco }}$ and the dijet mass $m_{j j}$, representing the mass of the hadronically decaying $W$ boson. We then discuss the results of applying these reconstruction techniques. Remember that by itself $m_{t}^{\text {reco }}$ is not an event-by-event measurement of the top quark mass; rather it is a quantity whose distribution in the data will be compared with simulated samples to extract the top quark mass (see Sec. V). Similarly, the distribution of $m_{j j}$ will be used to constrain the calibration of the jet energy scale in the reconstructed events.

Throughout the mass reconstruction, each event is assumed to be a $t \bar{t}$ event decaying in the lepton + jets channel, and the four leading jets are assumed to correspond to the four quarks from the top and $W$ decays. First, the measured four-vectors for the jets and lepton in the event are corrected for known effects, and resolutions are assigned where needed. Next, for the top quark mass reconstruction, a $\chi^{2}$ fit is used to extract the reconstructed mass, so that each event has a particular value of $m_{t}^{\text {reco }}$ and a corresponding $\chi^{2}$ value. Some events are discarded from the event sample when their minimized $\chi^{2}$ exceeds a cut value. Meanwhile, for the dijet mass reconstruction, the invariant mass $m_{j j}$ is calculated for each pair of jets without $b$ tags among the leading four jets.

\section{A. Inputs to the mass reconstruction}

The $\chi^{2}$ fit takes as input the four-vectors of the jets and lepton identified in the event. All known corrections are applied to these 4-vectors, and Gaussian uncertainties are computed for the transverse momenta, since they will be permitted to vary in the fit. The treatment of the neutrino four-vector is more complicated, since the $\mathscr{E}_{T}$ is a derived quantity and does not have an uncertainty independent of the other measured values. The $\chi^{2}$ includes instead information about a related fundamental quantity, the unclustered energy, which is described below.

\section{Jet inputs}

The corrections made to the jet four-vectors are described in detail in Sec. III A. To summarize, a series of corrections are applied to the jet energies in order to determine the energy of the quark corresponding to each jet. The jet angles are relatively well measured and are fixed in the kinematic fit. The final step of the jet corrections is the $t \bar{t}$-specific correction that treats separately $b$ jets and jets from the $W$ decay and, in addition, provides for the $p_{T}$ of each jet a resolution that is used in the $\chi^{2}$ expression.

\section{Lepton inputs}

The electron four-vector has energy determined by its electromagnetic calorimeter cluster and angles defined by the associated track. The electron energy is corrected for differences in the calorimeter response depending on where in the tower face the electron enters. The electron mass is set to zero, and the angles are taken as perfectly measured quantities. The transverse momentum $\left(p_{T}^{e}=\right.$ $p \sin \theta)$ of the electron is assigned an uncertainty of

$$
\frac{\sigma_{p_{T}^{e}}}{p_{T}^{e}}=\sqrt{\left(\frac{0.135}{\sqrt{p_{T}^{e}[\mathrm{GeV} / c]}}\right)^{2}+(0.02)^{2}} .
$$

The muon four-vector uses the three-vector of the associated track, also with a mass of zero. Track curvature corrections due to chamber misalignment are applied. The angles and mass are given no uncertainty; the transverse momentum has an uncertainty of

$$
\frac{\sigma_{p_{T}^{\mu}}^{\mu}}{p_{T}^{\mu}}=0.0011 \cdot p_{T}^{\mu}[\mathrm{GeV} / c] \text {. }
$$

The uncertainties on measured electron and muon transverse momenta are obtained from studies of leptonic $Z^{0}$ decays.

\section{Neutrino inputs: Unclustered energy}

The neutrino in a $t \bar{t}$ event is not observed; its presence is inferred by an imbalance in the observed transverse momentum. Therefore, rather than treating the neutrino fourvector as an independent input to the $\chi^{2}$ fit, the measured quantities, as varied in the fit, are used to dynamically calculate the neutrino transverse momentum.

All of the transverse energy in the calorimeter (towers with $|\eta|<3.6)$ that is not associated with the primary lepton or one of the leading four jets is considered "unclustered energy." For towers clustered into a jet that has $E_{T}>8 \mathrm{GeV}$ and $|\eta|<2.0$, but that is not one of the leading four jets, the tower momenta are replaced with the jet momentum after the generic jet corrections described in Sec. III A 2. The rest of the tower momenta are multiplied by a scale factor of 1.4 , which is the estimated generic correction factor for $8 \mathrm{GeV}$ jets. Finally, the unclustered energy includes the energy attributed to enter into the leading four jets from the underlying event, and excludes the energy thought to fall outside the jet cones of the leading four jets. This avoids double-counting of energy that is included in the leading four jet energies after all 
corrections. Each transverse component of the unclustered energy $\left(p_{x}^{U E}, p_{y}^{U E}\right)$ is assigned an uncertainty of $0.4 \sqrt{\sum E_{T}^{\text {uncl }}}$, where $\sum E_{T}^{\text {uncl }}$ is the scalar sum of the transverse energy excluding the primary lepton and leading four jets. The uncertainty comes from studies of events with no real missing energy and no hard jet activity.

The unclustered energy is the observed quantity and the input to the $\chi^{2}$ fit, but it is related to the missing energy through the other measured physics objects in the event, since the $p \bar{p}$ system has total transverse momentum close to 0 . The neutrino transverse momentum $p_{T}^{\nu}$ is calculated at each step of the fit, using the fitted values of lepton, jet, and unclustered transverse energies:

$$
\vec{p}_{T}^{\nu}=-\left(\vec{p}_{T}^{\ell}+\sum \vec{p}_{T}^{\mathrm{jet}}+\vec{p}_{T}^{U E}\right) .
$$

Note that this quantity, used in the mass fitting procedure, is different from the missing energy described in Sec. II D and used in event selection, where simpler calorimeter energy corrections are used.

Although other treatments of the unclustered energy and missing energy can be motivated, the $\mathscr{E}_{T}$ calculation does not have a large effect on the results of the $\chi^{2}$ fit. Various other approaches to correcting the unclustered energy and assigning resolution were tried, and no changes had any significant effect on the reconstructed top quark mass resolution.

The mass of the neutrino is fixed at zero, and the longitudinal momentum, $p_{z}^{\nu}$, is a free (unconstrained) parameter in the fit. The initial value of $p_{z}^{\nu}$ is calculated using the initial value of the lepton four-vector and the initial $p_{T}^{\nu}$, assuming that they arise from a $W$ boson at the nominal pole mass. Since these conditions yield a quadratic equation, there are in general two solutions for the $p_{z}^{\nu}$; a separate $\chi^{2}$ fit is done with each solution used as the initial value of $p_{z}^{\nu}$. When the solutions are imaginary, the real part $\pm 20 \mathrm{GeV} / c$ are the two values of $p_{z}^{\nu}$ used to initialize the fit.

\section{B. Event $\chi^{2}$ fit}

Given the inputs described above, the event-by-event fit for the reconstructed top quark mass proceeds as follows. MINUIT is used to minimize a $\chi^{2}$ where $m_{t}^{\text {reco }}$ is a free parameter. For each event, the $\chi^{2}$ is minimized once for each possible way of assigning the leading four jets to the four quarks from the $t \bar{t}$ decay. Since the two $W$ daughter jets are indistinguishable in the $\chi^{2}$ expression, the number of permutations is $\frac{4 !}{2}=12$. In addition, there are two solutions for the initial value of the neutrino longitudinal momentum, so the minimization is performed a total of 24 times for each event. When $b$ tags are present, permutations that assign a tagged jet to a light quark at parton level are rejected. In the case of single-tagged events, the number of allowed permutations is six, and for double-tagged events it is two. In the rare cases when an event has three $b$ tags, two of the tagged jets must be assigned to $b$ quarks. We use the reconstructed top quark mass from the permutation with the lowest $\chi^{2}$ after minimization.

The $\chi^{2}$ expression has terms for the uncertainty on the measurements of jet, lepton, and unclustered energies, as well as terms for the kinematic constraints applied to the system:

$$
\begin{aligned}
\chi^{2}= & \sum_{i=\ell, \text { jets }} \frac{\left(p_{T}^{i, \text { fit }}-p_{T}^{i, \text { meas }}\right)^{2}}{\sigma_{i}^{2}}+\sum_{j=x, y} \frac{\left(p_{j}^{U E, \text { fit }}-p_{j}^{U E, \text { meas }}\right)^{2}}{\sigma_{U E}^{2}} \\
& +\frac{\left(M_{\ell \nu}-M_{W}\right)^{2}}{\Gamma_{W}^{2}}+\frac{\left(M_{j j}-M_{W}\right)^{2}}{\Gamma_{W}^{2}} \\
& +\frac{\left(M_{b \ell \nu}-m_{t}^{\text {reco }}\right)^{2}}{\Gamma_{t}^{2}}+\frac{\left(M_{b j j}-m_{t}^{\text {reco }}\right)^{2}}{\Gamma_{t}^{2}}
\end{aligned}
$$

The first term constrains the $p_{T}$ of the lepton and four leading jets to their measured values within their assigned uncertainties; the second term does the same for both transverse components of the unclustered energy. In the remaining four terms, the quantities $M_{\ell \nu}, M_{j j}, M_{b \ell_{\nu}}$, and $M_{b j j}$ refer to the invariant mass of the sum of the fourvectors denoted in the subscripts. For example, $M_{\ell \nu}$ is the invariant mass of the sum of the lepton and neutrino fourvectors. $M_{W}$ is the pole mass of the $W$ boson, $80.42 \mathrm{GeV} / c^{2}$ [28], and $m_{t}^{\text {reco }}$ is the free parameter for the reconstructed top quark mass used in the minimization. $M_{j j}$ is a quantity computed in the kinematic fit and should not be confused with $m_{j j}$, the measured dijet mass used to constrain $\Delta_{\mathrm{JES}}$. The fit is initialized with $m_{t}^{\text {reco }}=$ $175 \mathrm{GeV} / c^{2} . \Gamma_{W}$ and $\Gamma_{t}$ are the total width of the $W$ boson and the top quark. In order to use the $\chi^{2}$ formalism, the $W$ and top Breit-Wigner lineshapes are modeled with Gaussian distributions, using the Breit-Wigner full width at half maximum as the Gaussian sigma. $\Gamma_{W}$ is $2.12 \mathrm{GeV}$ [28], and $\Gamma_{t}$ is $1.5 \mathrm{GeV}$ [29]. Thus these terms provide constraints such that the $W$ masses come out correctly, and the $t$ and $\bar{t}$ masses come out the same (modulo the BreitWigner distribution, here modeled by a Gaussian, in both cases).

The jet-quark assignment (and $p_{z}^{\nu}$ solution) with the lowest $\chi^{2}$ after minimization is selected for each event. The $\chi^{2}$ of this combination is denoted $\chi_{\min }^{2}$ (or just $\chi^{2}$ when the context is unambiguous), and the requirement $\chi_{\min }^{2}<9$ is imposed. The expected statistical uncertainty on the top quark mass does not change much over a wide range of the value of the cut, even when it is varied independently for the four event types. The value of the cut chosen is close to the minimum of expected top quark mass uncertainty.

\section{Dijet mass and jet energy scale}

We calculate the dijet masses used to constrain $\Delta_{\mathrm{JES}}$ in the same data sample used to reconstruct the observed top 
quark mass, with the exception that there is no $\chi^{2}$ requirement on the jet-quark assignments under consideration. The imposition of the $\chi^{2}$ requirement would impose a bias in the dijet masses being considered and therefore reduce the sensitivity of the dijet mass distribution to $\Delta_{\text {JES }}$. We calculate the dijet masses directly from the measured jet four-vectors without the use of a kinematic fit, considering all jet-quark assignments in each event for any of the leading four jets that are not $b$-tagged. Monte Carlo studies have shown that the sensitivity of the dijet mass distribution to the $\Delta_{\text {JES }}$ parameter is maximized by considering all dijet mass combinations that do not involve a $b$-tagged jet in each event. The number of possible assignments ranges from one (for events with two $b$ tags) to six (for events with no $b$ tags).

\section{Mass reconstruction results}

Typical reconstructed top quark mass distributions for signal Monte Carlo $\left(\mathrm{M}_{\text {top }}=178 \mathrm{GeV} / \mathrm{c}^{2}\right)$ are shown for the four event categories as the light histograms in Fig. 7. Each event in the sample that passes both event selection and the $\chi^{2}$ cut contributes exactly one entry to these histograms. The distributions peak near the generated mass of $178 \mathrm{GeV} / c^{2}$. But there is not an exact correspondence between the generated mass and the mean or peak position of the reconstructed mass. Differences can arise when ISR/
FSR jets are selected instead of the $t \bar{t}$ decay products; even with the correct jets, the fit may choose the wrong jet-quark assignment. In particular, the broader shape, beneath the relatively sharp peak at $178 \mathrm{GeV} / c^{2}$, comprises events where an incorrect permutation has been chosen in the fit. The dark histograms in the same figure show the reconstructed mass distributions for events where the four leading jets correspond to the four quarks from $t \bar{t}$ decay and where the correct jet-quark assignment is chosen by the fit. These histograms have much smaller tails than the overall distributions and account for $47 \%$ of the 2-tag sample, $28 \%$ of the $1-\operatorname{tag}(\mathrm{T})$ sample, $18 \%$ of the $1-\operatorname{tag}(\mathrm{L})$, and $20 \%$ of the 0 -tag category.

The corresponding dijet mass distributions for the $W$ boson reconstruction are shown in Fig. 8 for the four subsamples. Each event contributes 1, 3, or 6 entries to the distributions, depending on the number of $b$ tags. One sees a clear $W$ boson mass signal, with a peak near the nominal $W$ boson mass of $80 \mathrm{GeV} / c^{2}$. The peak becomes more evident with increasing numbers of $b$-tagged jets in the event, a consequence of the decreasing number of combinations for $W$ boson jet daughters.

Some results of the mass reconstruction on Monte Carlo $t \bar{t}$ signal $\left(\mathrm{M}_{\mathrm{top}}=178 \mathrm{GeV} / c^{2}\right)$ and background samples are given in Table III. The four subsamples have significantly different $m_{t}^{\text {reco }}$ and $m_{j j}$ shapes for $t \bar{t}$ signal and background, as evidenced by their reconstructed mass
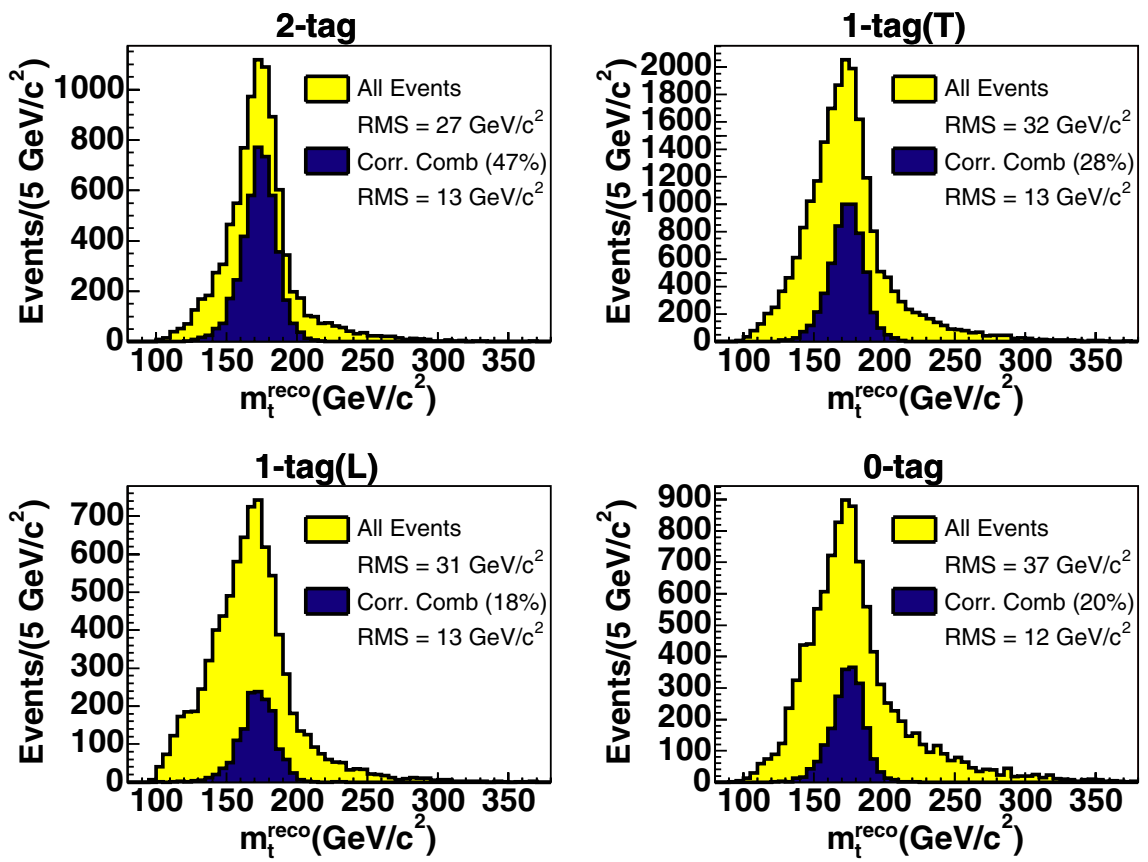

FIG. 7 (color online). The light histograms show the reconstructed top quark mass distribution for the $178 \mathrm{GeV} / c^{2}$ HERWIG $t \bar{t}$ sample at the nominal jet energy scale. Overlaid are darker histograms of the reconstructed mass distributions using the subset of events for which the leading four jets are matched (within $\Delta R=0.4$ ) to the four quarks from the $t \bar{t}$ decay and the correct jet-quark assignment has the lowest $\chi^{2}$. Distributions are shown for 2-tag (upper left), 1-tag(T) (upper right), 1-tag(L) (lower left), and 0-tag (lower right) events. 

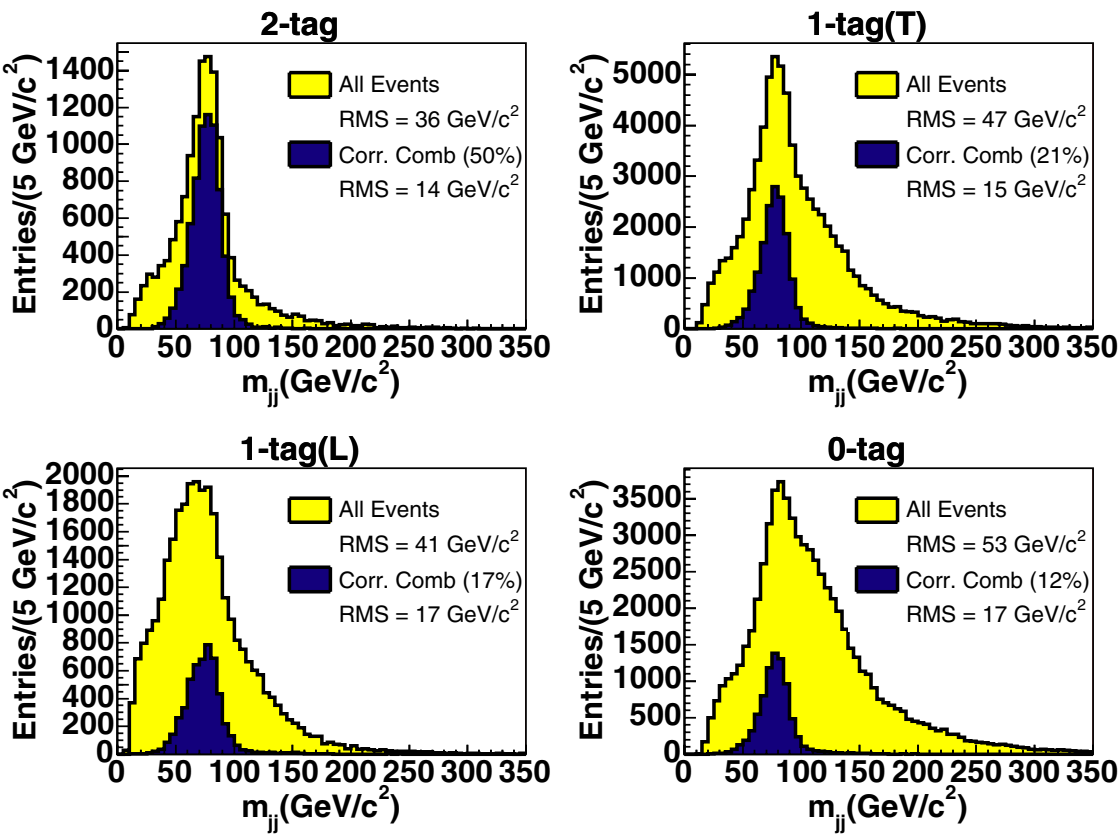

FIG. 8 (color online). The reconstructed dijet mass distributions for the $178 \mathrm{GeV} / c^{2}$ HERWIG $t \bar{t}$ sample at the nominal jet energy scale. Overlaid are darker histograms of the reconstructed mass distributions using the subset of events for which the leading four jets include two jets matched (within $\Delta R=0.4$ ) to the two quarks from the hadronic $W$ decay and plotting just the invariant mass of those two jets. Distributions are shown for 2-tag (upper left), 1-tag(T) (upper right), 1-tag(L) (lower left), and 0-tag (lower right) events.

mean and root mean square (RMS) values. The $\chi^{2}$ cut efficiency is lowest for 2-tag events, especially for the background processes, because there are fewer allowed jet-quark assignments and thus fewer chances to pass the $\chi^{2}$ cut. The efficiencies for signal events vary only weakly with the generated top quark mass and for the purposes of this analysis are assumed to be constant. The means of the background reconstructed mass distributions are primarily driven by the jet cuts (see Table I).

TABLE III. Monte Carlo samples of $t \bar{t}$ signal and of background processes with the expected relative weights are run through the $\chi^{2}$ mass fitter. For signal and background in each of the four event categories, the table shows the efficiency of the $\chi^{2}$ cut and the mean and RMS of the resulting reconstructed mass distributions. The signal sample has $\mathrm{M}_{\mathrm{top}}=178 \mathrm{GeV} / \mathrm{c}^{2}$, and the nominal jet energy scale is used for all events.

\begin{tabular}{lccccc}
\hline \hline Sample & \multirow{2}{*}{$\begin{array}{c}\text { 2 cut } \\
\text { Description }\end{array}$} & $\begin{array}{c}\text { reco } \\
\text { eff. }\end{array}$ & Mean & RMS & \multicolumn{2}{c}{$m_{j j}\left(\mathrm{GeV} / c^{2}\right)$} \\
Signal & & & & & \\
2-tag & 0.65 & 173.9 & 26.6 & 76.8 & 34.5 \\
1-tag(T) & 0.85 & 174.0 & 31.8 & 98.1 & 49.1 \\
1-tag(L) & 0.80 & 167.4 & 30.8 & 77.8 & 41.7 \\
0-tag & 0.91 & 179.3 & 36.9 & 112.6 & 57.9 \\
\hline Background & & & & & \\
2-tag & 0.38 & 160.2 & 35.1 & 77.2 & 53.3 \\
1-tag(T) & 0.73 & 166.4 & 42.2 & 95.8 & 59.7 \\
1-tag(L) & 0.71 & 153.7 & 37.3 & 67.7 & 46.3 \\
0-tag & 0.83 & 182.6 & 46.5 & 114.0 & 69.3 \\
\hline \hline
\end{tabular}
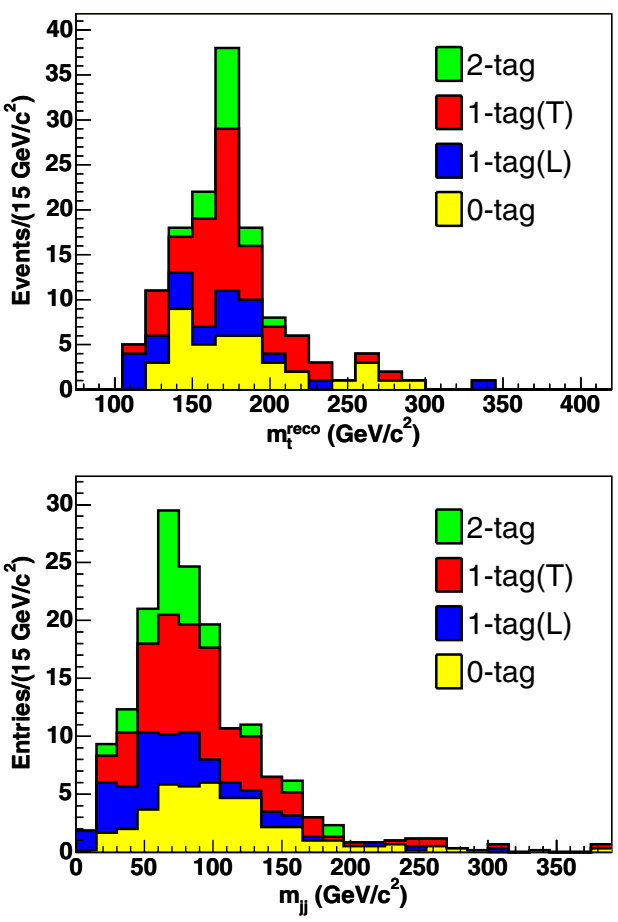

FIG. 9 (color online). The top histogram shows the reconstructed top quark mass $m_{t}^{\text {reco }}$, and the bottom histogram shows the dijet reconstructed mass $m_{j j}$, with events from the four subsamples represented by separate stacked histograms. In the $m_{j j}$ plot, each event has a different number of jet pairs, depending on the number of $b$ tags in the event, but the entries are weighted so that the total contribution from each event is one unit. The highest $m_{j j}$ bin contains overflow entries. 

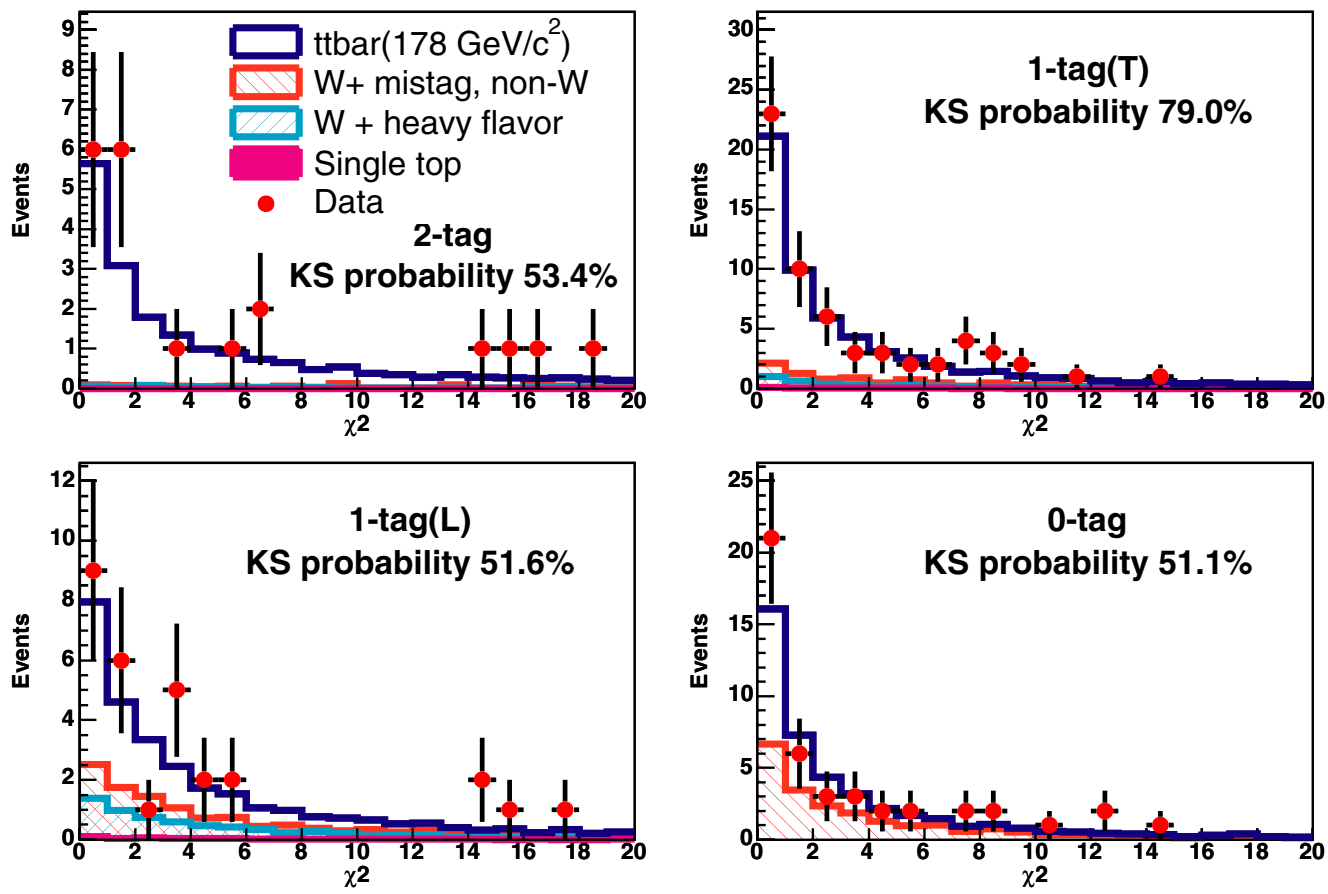

FIG. 10 (color online). The $\chi^{2}$ distribution is shown for data events and for signal and background simulated events in the expected ratio. Distributions are shown for 2-tag (upper left), 1-tag(T) (upper right), 1-tag(L) (lower left), and 0-tag (lower right) events.

The reconstructed top quark and dijet mass distributions for the 165 events found in the data can be seen in Fig. 9. These events consist of both $t \bar{t}$ signal and background events. Figure 10 shows distributions of $\chi^{2}$ values from the top quark mass reconstruction in data and simulated events, where the distributions from simulation contain the expected mixtures of signal and background events. Kolmogorov-Smirnov tests, with probability normalized using many trial distributions randomly selected from the Monte Carlo predictions, show that the distributions agree well, indicating that kinematic quantities and resolutions are correctly simulated.

\section{TOP QUARK MASS FITTING}

The distribution of reconstructed mass (either $m_{t}^{\text {reco }}$ or $m_{j j}$ ) for a particular top quark mass (or background process) and shift in the jet energy scale is referred to as a template. We compare the reconstructed top quark mass distribution and the dijet mass distribution from data to the Monte Carlo templates to measure simultaneously the top quark mass and the jet energy scale shift. First, probability density functions (p.d.f.'s) for the reconstructed top quark and dijet masses are determined for signal events and background events in each subsample by fitting a functional form to the corresponding templates; the signal p.d.f.'s depend on the top quark mass and jet energy scale. The shift in jet energy scale is given by $\Delta_{\text {JES }}$, which is the relative shift in units of the nominal uncertainty in the jet energy scale derived from the extrinsic calibration proce- dures (Sec. III B). Although the jet energy scale uncertainty varies with jet momentum and pseudorapidity, a one unit shift in the $\Delta_{\text {JES }}$ parameter is approximately equivalent to a $3 \%$ shift in the jet energy scale for jets in $t \bar{t}$ events. We perform an unbinned likelihood fit to determine the values of $\mathrm{M}_{\text {top }}$ and $\Delta_{\mathrm{JES}}$ that best describe the data. At the end of this section, we describe a number of checks of the method using simulated events.

\section{A. Parameterization of signal and background shapes}

Since templates are available only at discrete values of top quark mass and jet energy scale, the signal reconstructed mass distributions in each subsample are parameterized by a flexible functional form as a function of $\mathrm{M}_{\text {top }}$ and $\Delta_{\text {JES }}$ in order to smooth the distributions and interpolate between the templates.

For background events, the parameterization has no dependence on top quark mass or jet energy scale; a single p.d.f. is used to describe each background reconstructed mass shape in each subsample. In principle, a shift in the jet energy scale can change the shape of the background templates. However, we have determined from studies of the background that the shape of the background templates are insensitive to shifts in the jet energy scale. Rather, the overall rate of background events does show some sensitivity to the jet energy response, and this uncertainty is incorporated into the uncertainty in the rate of background events in the sample. 
The same parameterizations are used for both $m_{t}^{\text {reco }}$ and $m_{j j}$ signal p.d.f.'s, although of course the fitted parameters are different. In the case of the background, different functional forms are required to fit well the $m_{t}^{\text {reco }}$ and $m_{j j}$ templates.

\section{Signal shape parameterization}

Signal templates are produced using sets of Monte Carlo samples with the input top quark mass at $2.5-5 \mathrm{GeV} / c^{2}$ intervals from $130 \mathrm{GeV} / c^{2}$ to $230 \mathrm{GeV} / c^{2}$ and the jet energy scale shift varying from $-3.0 \sigma_{c}$ to $+3.0 \sigma_{c}$ in steps of 0.5. Examples of the template shapes from each event category are given in Fig. $7\left(m_{t}^{\text {reco }}\right)$ and Fig. $8\left(m_{j j}\right)$. Table IV shows the evolution of the mean, most probable value, and RMS of the reconstructed top quark mass templates as a function of top quark mass using selected generated mass samples and the nominal jet energy scale.

We derive from these distributions parameterized templates that are a smoothly varying function of top quark mass and jet energy scale. For any given $\mathrm{M}_{\text {top }}$ and $\Delta_{\mathrm{JES}}$, the probability to observe a particular reconstructed mass is specified by a function consisting of two Gaussians - intended to account for the well-reconstructed quantities plus a gamma distribution - intended to account for cases where the incorrect jets are used to reconstruct the top quark or $W$ masses. The 9 parameters necessary to specify this combination of functions are themselves assumed to depend linearly on $\mathrm{M}_{\mathrm{top}}$ and $\Delta_{\mathrm{JES}}$, so that the full set of p.d.f.'s is specified by 27 parameters. This assumed functional form works well in the limited range of top quark

TABLE IV. The evolution of the $m_{t}^{\text {reco }}$ template parameters is demonstrated using selected signal Monte Carlo samples with generated top quark mass of $145 \mathrm{GeV} / c^{2}, 165 \mathrm{GeV} / c^{2}$, $185 \mathrm{GeV} / c^{2}$, and $205 \mathrm{GeV} / c^{2}$, with the nominal jet energy scale. The mean, most probable value (MPV), and RMS of the template are given for each subsample in each generated mass. The mean and MPV of the templates are driven by the jet $E_{T}$ cuts, and the widths are dominated by the fraction of events with correct jet-quark assignments.

\begin{tabular}{lccccc}
\hline \hline & $\mathrm{M}_{\text {top }}$ & 2-tag & 1-tag(T) & 1-tag(L) & 0-tag \\
\hline & 145 & 151.5 & 155.1 & 147.1 & 163.9 \\
Mean & 165 & 164.5 & 166.5 & 158.8 & 174.3 \\
$\left(\mathrm{GeV} / c^{2}\right)$ & 185 & 178.2 & 179.1 & 171.1 & 185.3 \\
& 205 & 193.5 & 190.5 & 182.7 & 194.2 \\
\hline & 145 & 144.5 & 144.0 & 140.7 & 145.0 \\
$\mathrm{MPV}$ & 165 & 163.8 & 159.5 & 156.5 & 159.3 \\
$\left(\mathrm{GeV} / c^{2}\right)$ & 185 & 179.9 & 178.1 & 171.7 & 179.5 \\
& 205 & 198.5 & 194.7 & 185.4 & 193.9 \\
\hline & 145 & 25.1 & 31.7 & 28.5 & 39.2 \\
$\mathrm{RMS}$ & 165 & 24.8 & 31.8 & 28.6 & 39.1 \\
$\left(\mathrm{GeV} / c^{2}\right)$ & 185 & 27.1 & 32.3 & 32.1 & 37.7 \\
& 205 & 28.6 & 33.6 & 34.1 & 37.7 \\
\hline \hline
\end{tabular}

masses and jet energy scales considered; as an example, letting the 9 parameters have quadratic dependence on $\mathrm{M}_{\text {top }}$ or $\Delta_{\mathrm{JES}}$ does not improve the fit. Thus the parameterization is as follows:

$$
\begin{aligned}
P_{s}\left(m ; \mathrm{M}_{\mathrm{top}}, \Delta_{\mathrm{JES}}\right)= & \alpha_{7} \cdot \frac{\alpha_{2}^{1+\alpha_{1}}}{\Gamma\left(1+\alpha_{1}\right)} \cdot\left(m-\alpha_{0}\right)^{\alpha_{1}} \\
& \cdot \exp \left(-\alpha_{2}\left(m-\alpha_{0}\right)\right)+\alpha_{8} \cdot \frac{1}{\alpha_{4} \sqrt{2 \pi}} \\
& \cdot \exp \left(\frac{-\left(m-\alpha_{3}\right)^{2}}{2 \alpha_{4}^{2}}\right)+\left(1-\alpha_{7}\right. \\
& \left.-\alpha_{8}\right) \cdot \frac{1}{\alpha_{6} \sqrt{2 \pi}} \cdot \exp \left(\frac{-\left(m-\alpha_{5}\right)^{2}}{2 \alpha_{6}^{2}}\right)
\end{aligned}
$$

where

\begin{tabular}{|c|c|c|c|}
\hline 2-tag & $1-\operatorname{tag}(\mathrm{T})$ & $1-\operatorname{tag}(\mathrm{L})$ & 0 -tag \\
\hline \multicolumn{4}{|c|}{$\chi^{2} /$ n.d.o.f. for reconstructed top quark mass [Eq. (5.1)] } \\
\hline \multicolumn{4}{|c|}{$\chi^{2} /$ n.d.o.f. for dijet mass [Eq. (5.1)] } \\
\hline
\end{tabular}

TABLE V. The $\chi^{2}$ and number of degrees of freedom are given for the signal parameterization fits in each of the four subsamples.

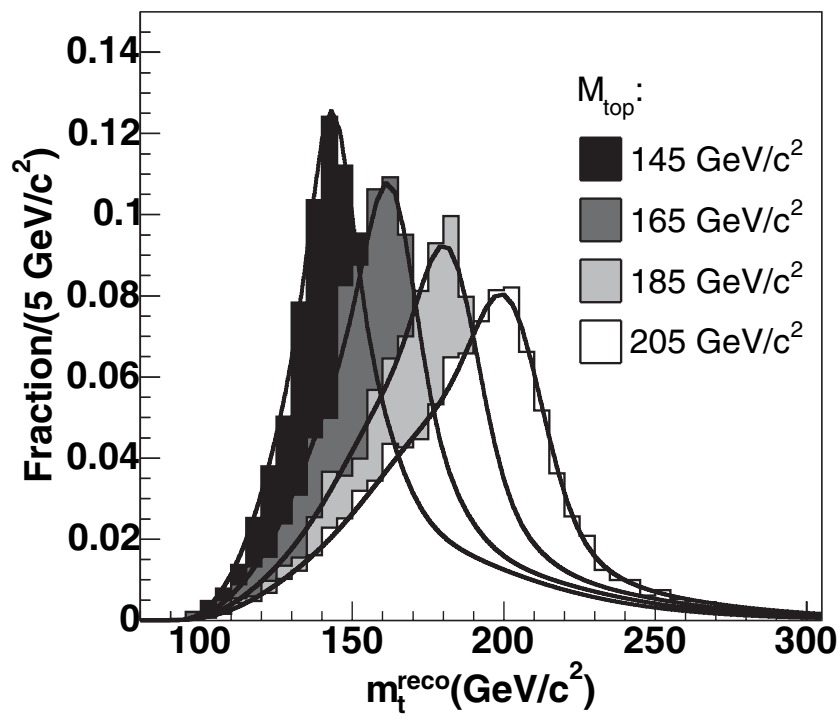

FIG. 11. Four $m_{t}^{\text {reco }}$ signal templates for the 1-tag(T) sample are shown, with top quark masses ranging from $145 \mathrm{GeV} / c^{2}$ to $205 \mathrm{GeV} / c^{2}$ and with $\Delta_{\mathrm{JES}}$ set to 0 . Overlaid are the fitted parameterizations at each generated mass, taken from the full parameterization given in Eq. (5.1). 


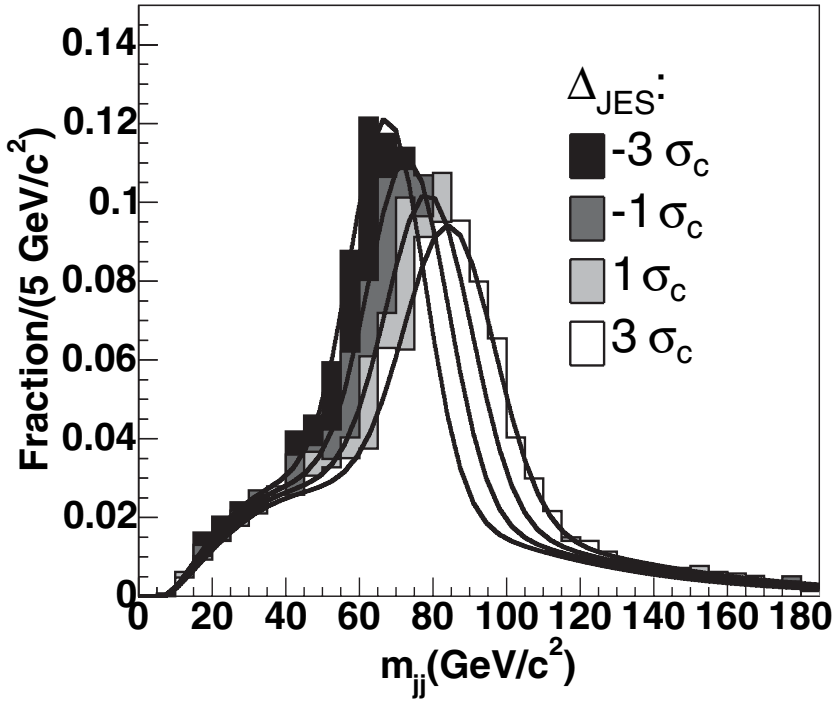

FIG. 12. Four $m_{j j}$ signal templates for the 2-tag sample are shown, with $\Delta_{\mathrm{JES}}$ values ranging from $-3 \sigma_{c}$ to $3 \sigma_{c}$ and with the top quark mass set to $180 \mathrm{GeV} / c^{2}$. Overlaid are the fitted parameterizations at each value of the jet energy scale shift, taken from the full parameterization given in Eq. (5.1).

$$
\alpha_{i}=p_{i}+p_{i+9} \cdot\left(\mathrm{M}_{\mathrm{top}}-175\right)+p_{i+18} \cdot\left(\Delta_{\mathrm{JES}}\right) .
$$

The variable $m$ in $\mathrm{GeV} / c^{2}$ refers to the reconstructed top quark or dijet mass, $\mathrm{M}_{\text {top }}$ in $\mathrm{GeV} / c^{2}$ refers to the top quark mass, and $\Delta_{\mathrm{JES}}$ refers to the shift in the jet energy scale from that determined by our calibrations. These template parameterizations are normalized so that, for a given top quark mass $\mathrm{M}_{\text {top }}$ and jet energy scale shift $\Delta_{\mathrm{JES}}$, the integral over all reconstructed masses $m$ is unity.

A binned likelihood fit is used to determine the 27 parameter values both for the $m_{t}^{\text {reco }}$ templates and for the $m_{j j}$ templates. The $\chi^{2}$ is calculated between the MC samples and the prediction from the fit, after rebinning to ensure that each bin has at least five predicted events. The resulting $\chi^{2}$ values are given in Table $\mathrm{V}$, along with the number of degrees of freedom. Clearly the corresponding probabilities are small, not surprising considering the limited flexibility of the functional form and the large statistics of the templates. But the method check and calibration of Sec. V C show that the disagreement between the templates and the parameterizations is not large enough to have a significant effect on the measurement.

In Fig. 11, four signal templates at varying generated masses are shown overlaid with the fitted parameterization evaluated at each mass. This figure exhibits the changing shape of the reconstructed mass templates as a function of top mass. Figure 12 shows the $m_{j j}$ templates with varying jet energy scale, overlaid with the fitted parameterization. One sees that the location of the $W$ boson peak is sensitive to the jet energy scale.

\section{Background shape parameterization}

Monte Carlo simulations of the various processes listed in Sec. II E are used to model the reconstructed top quark mass shape and dijet mass shape for background processes. When possible, a single large-statistics sample is used to represent several background processes that have similar template shapes.

For the tagged backgrounds, the $W+$ heavy flavor processes $(W b \bar{b}, W c \bar{c}, W c)$ all have similar reconstructed mass shapes, as shown in Fig. 13 for $m_{t}^{\text {reco }}$ in the 1-tag(T) sample, and thus are all modeled with a high-statistics $W b \bar{b}$ simulated sample. $W W$ and $W Z$ events, a negligible contribution to the total expected background, are also included in this category. The shapes for the three subsamples with tagged events are found by reconstructing the simulated events exactly as is done for the data and signal Monte Carlo. Similarly, the simulated $s$ - and $t$-channel single top quark events are used to obtain corresponding mass templates.

The mass templates for the $W+$ jets backgrounds in the tagged subsamples, i.e. "mistags," are not obtained using the Monte Carlo $b$ tagging, which is not expected to model well the rate or kinematic dependences of fake tags. Instead, a mistag matrix, derived from the data, is used to give the probability for a jet to be falsely tagged as a function of its $E_{T}, \phi, \eta$, number of tracks, and the $\Sigma E_{T}$ for all jets in the event. Then for each simulated event $(W+4$ partons, generated by ALPGEN and showered by HERWIG), every possible tag configuration on the leading four jets is considered. For every tag configuration, the fit with lowest $\chi^{2}$ among the jet-quark assignments consistent with the assumed tags is selected, and the appropriate mass template is filled with a weight corresponding to the probability of observing that set of tags. The result is a weighted template for the mistag backgrounds.

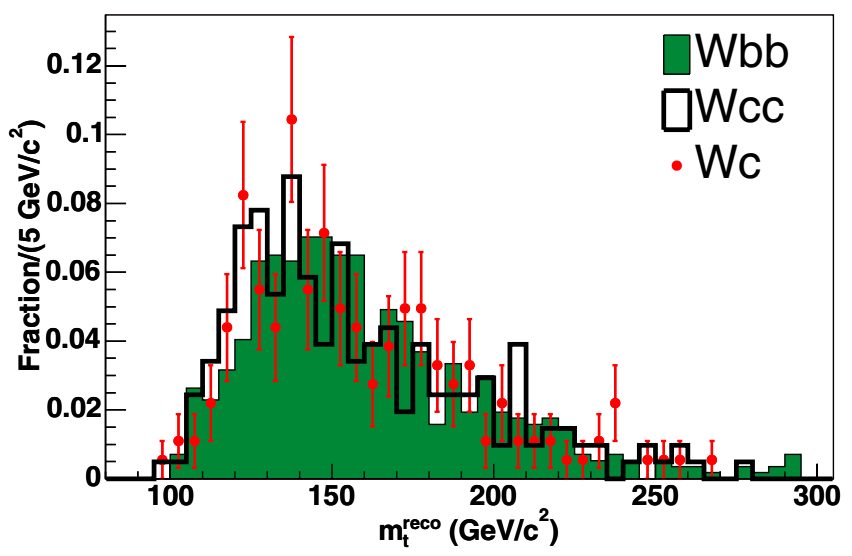

FIG. 13 (color online). The templates for $W c \bar{c}$ and $W c$ background processes are compared to the high-statistics $W b \bar{b}$ template for all tagged events. The agreement is good in both cases, so the $W b \bar{b}$ template is used to represent all $W+$ h.f. processes. 
The backgrounds that are least amenable to Monte Carlo modeling arise from QCD background events, i.e. events with no real $W$ to produce the isolated lepton and $\mathbb{E}_{T}$. These events are difficult to simulate but can be studied by selecting events in the data with nonisolated leptons, which are enriched in this type of background, but kinematically similar to events chosen in the default selection.

The mass reconstruction described in Sec. IV is expected to produce similar results for QCD background events and $W+$ jets background events. This is because the leptonic $W$ system, in which these types of events differ, does not have a strong effect on the mass reconstruction, since $\mathbb{E}_{T}$ is poorly measured and since the $W$ mass is constrained in the $\chi^{2}$ expression. In the kinematic properties of the jets, to which the mass reconstruction is very sensitive, these two types of events are similar since in both cases the jets arise from hard QCD radiation.

Indeed, within the limited statistics available, the reconstructed mass distributions of the QCD-enriched data events are consistent with those of simulated $W+$ jets events. Given these similarities, the $W+$ jets reconstructed mass templates are used also for the expected contributions from QCD for both the reconstructed top quark mass and the dijet invariant mass. An additional check, treating the subset of QCD events where the primary lepton is a jet misidentified as an electron, is performed using a large QCD-dominated data set with at least five jets. One jet with a large fraction of its energy in the electromagnetic calorimeter is assigned to "fake" an electron, and the mass reconstruction proceeds under that hypothesis. Very good agreement is found between the reconstructed mass distributions of these events and those of simulated $W+$ jets events. A systematic uncertainty on the background modeling (see Sec. VIIC) is assigned using the differences between the templates obtained from these three samples: $W+$ jets events, events with nonisolated leptons, and events with one jet assigned to fake an electron.

The background for the 0-tag subsample is treated separately from the others. The dominant process is $W+$ jets, with a smaller $(\sim 20 \%)$ contribution expected from non- $W$ (QCD) events. Since we model the reconstructed mass of QCD events using $W+$ jets events, the entire 0 -tag background shape comes from $W+4$ parton Monte Carlo events, simulated by ALPGEN and showered by HERWIG.

We do not allow the normalization of each background contribution to vary independently in the final likelihood fit. Instead, for each subsample, the templates from all background processes are combined in their expected ratios according to Table II. A single function is fitted to the combined background for each subsample and is used to describe the background shape in the final likelihood fit (Sec. V B). The overall background normalization for each subsample is then permitted to vary, within its constraint where applicable.

We determine the p.d.f.'s for the background reconstructed top quark mass templates using a parameterization similar in spirit to that of the signal, but simpler in form.
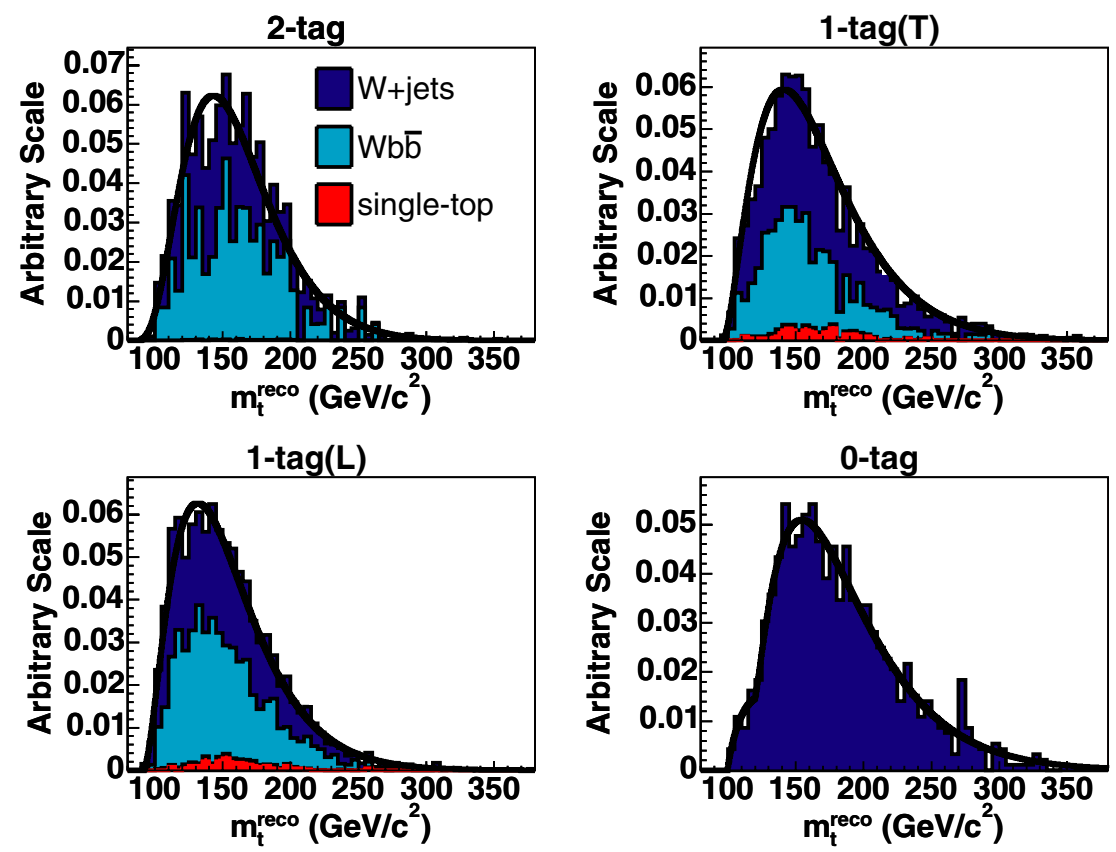

FIG. 14 (color online). Reconstructed top quark mass distributions of the combined backgrounds in each subsample. The contributions from different background templates are shown stacked; overlaid are the fitted curves [see Eq. (5.2) and (5.3)]. 

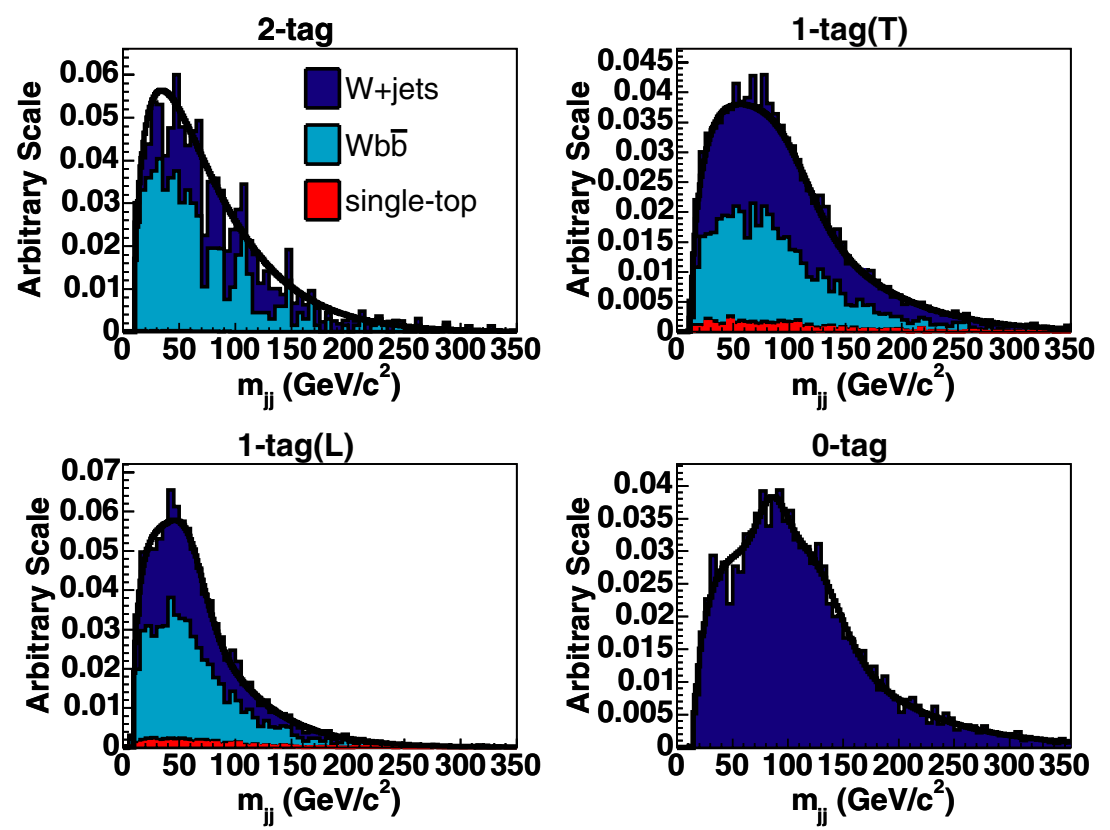

FIG. 15 (color online). Reconstructed dijet mass distributions of the combined backgrounds in each subsample. The contributions from different background templates are shown stacked; overlaid are the fitted curves [see Eqs. (5.4) and (5.5)].

First, there is no dependence on top quark mass or jet energy scale. Second, no narrow Gaussian peak is expected, so the full shape is modeled by the integrand of the gamma distribution. Specifically,

$$
\begin{aligned}
P_{b}\left(m_{t}^{\text {reco }}\right)= & \frac{p_{2}^{1+p_{1}}}{\Gamma\left(1+p_{1}\right)} \cdot\left(m_{t}^{\text {reco }}-p_{0}\right)^{p_{1}} \\
& \cdot \exp \left(-p_{2}\left(m_{t}^{\text {reco }}-p_{0}\right)\right) .
\end{aligned}
$$

In the case of the 0-tag background events, a slightly more sophisticated function is used to achieve a good fit:

$$
\begin{aligned}
P_{b}\left(m_{t}^{\text {reco }}\right)= & p_{6} \frac{p_{2}^{1+p_{1}}}{\Gamma\left(1+p_{1}\right)} \cdot\left(m_{t}^{\text {reco }}-p_{0}\right)^{p_{1}} \\
& \cdot \exp \left(-p_{2}\left(m_{t}^{\text {reco }}-p_{0}\right)\right)+\left(1-p_{6}\right) \\
& \times \frac{p_{5}^{1+p_{4}}}{\Gamma\left(1+p_{4}\right)} \cdot\left(m_{t}^{\text {reco }}-p_{3}\right)^{p_{4}} \\
& \cdot \exp \left(-p_{5}\left(m_{t}^{\text {reco }}-p_{3}\right)\right) .
\end{aligned}
$$

In the background events, the following parameterizations are used to fit the reconstructed dijet mass templates. For the background samples with $b$ tags,

$$
\begin{aligned}
P_{b}\left(m_{\mathrm{jj}}\right)= & \alpha_{5} \cdot \frac{\alpha_{2}^{1+\alpha_{1}}}{\Gamma\left(1+\alpha_{1}\right)} \cdot\left(m_{\mathrm{jj}}-\alpha_{0}\right)^{\alpha_{1}} \\
& \cdot \exp \left(-\alpha_{2}\left(m_{\mathrm{jj}}-\alpha_{0}\right)\right)+\left(1-\alpha_{5}\right) \\
& \cdot \frac{1}{\alpha_{4} \sqrt{2 \pi}} \cdot \exp \left(\frac{-\left(m_{\mathrm{jj}}-\alpha_{3}\right)^{2}}{2 \alpha_{4}^{2}}\right),
\end{aligned}
$$

and for the 0-tag sample,

$$
\begin{aligned}
P_{b}\left(m_{\mathrm{jj}}\right)= & \alpha_{7} \cdot \frac{\alpha_{2}^{1+\alpha_{1}}}{\Gamma\left(1+\alpha_{1}\right)} \cdot\left(m_{\mathrm{jj}}-\alpha_{0}\right)^{\alpha_{1}} \\
& \cdot \exp \left(-\alpha_{2}\left(m_{\mathrm{jj}}-\alpha_{0}\right)\right)+\alpha_{8} \cdot \frac{1}{\alpha_{4} \sqrt{2 \pi}} \\
& \cdot \exp \left(\frac{-\left(m_{\mathrm{jj}}-\alpha_{3}\right)^{2}}{2 \alpha_{4}^{2}}\right)+\left(1-\alpha_{7}-\alpha_{8}\right) \\
& \cdot \frac{1}{\alpha_{6} \sqrt{2 \pi}} \cdot \exp \left(\frac{-\left(m_{\mathrm{jj}}-\alpha_{5}\right)^{2}}{2 \alpha_{6}^{2}}\right) .
\end{aligned}
$$

The final background templates for the reconstructed top quark mass and dijet mass for the four subsamples are shown in Figs. 14 and 15, respectively, overlaid with the fitted parameterization.

\section{B. Likelihood fit for top quark mass}

The reconstructed mass distributions from data are simultaneously compared to the templates from signal and background sources using an unbinned extended likelihood fit. The likelihood includes free parameters for the number 
of expected signal events $n_{s}$ and background events $n_{b}$ in each subsample, and for the top quark pole mass $\mathrm{M}_{\text {top }}$ and the shift in jet energy scale $\Delta_{\mathrm{JES}}$. For each subsample, the likelihood is given by

$$
\mathcal{L}_{\text {sample }}=\mathcal{L}_{\text {shape }}^{m_{t}^{\text {reco }}} \times \mathcal{L}_{\text {shape }}^{m_{\text {ij }}} \times \mathcal{L}_{\text {nev }} \times \mathcal{L}_{\text {bg }},
$$

where

$$
\begin{aligned}
\mathcal{L}_{\text {shape }}^{m_{t}^{\text {reco }}}= & \prod_{k=1}^{N \chi^{2}} \frac{\left[\epsilon_{s} n_{s} P_{s}\left(m_{t, k}^{\mathrm{reco}} ; \mathrm{M}_{\mathrm{top}}, \Delta_{\mathrm{JES}}\right)+\epsilon_{b} n_{b} P_{b}\left(m_{t, k}^{\mathrm{reco}}\right)\right]}{\epsilon_{s} n_{s}+\epsilon_{b} n_{b}} ; \\
\mathcal{L}_{\text {shape }}^{m_{\mathrm{jj}}}= & \prod_{k=1}^{N \cdot C_{i}} \frac{n_{s} P_{s}\left(m_{\mathrm{jj}, k} ; \mathrm{M}_{\mathrm{top}}, \Delta_{\mathrm{JES}}\right)+n_{b} P_{b}\left(m_{\mathrm{jj}, k}\right)}{n_{s}+n_{b}} ; \\
\mathcal{L}_{\mathrm{nev}}= & \sum_{N_{s}+N_{b}=N} \operatorname{Pois}\left(N_{s} ; n_{s}\right) \times \operatorname{Pois}\left(N_{b} ; n_{b}\right) \\
& \times\left[\sum_{N_{s}^{\chi^{2}}+N_{b}^{\chi^{2}}=N^{\chi^{2}}}^{N_{s, b}^{\chi^{2}} \leq N_{s, b}} B\left(N_{s}^{\chi^{2}} ; N_{s}, \epsilon_{s}\right) B\left(N_{b}^{\chi^{2}} ; N_{b}, \epsilon_{b}\right)\right] ; \\
\mathcal{L}_{\mathrm{bg}}= & \exp \left(-\frac{\left(n_{b}-n_{b}^{0}\right)^{2}}{2 \sigma_{n_{b}}^{2}}\right) .
\end{aligned}
$$

The values $\epsilon_{s}$ and $\epsilon_{b}$ represent the efficiency of the $\chi^{2}$ cut for signal and background events, respectively, and are given in Table III. $N$ and $N^{\chi^{2}}$ are the number of events observed in the data before and after the $\chi^{2}$ cut. All other symbols are explained below.

The most important information on the top quark mass is provided by the products in $\mathcal{L}_{\text {shape }}^{m_{t}^{\text {reco }}}$ shape, the $k$ th term of which gives the probability of observing the $k$ th data event with reconstructed mass $m_{t, k}^{\text {reco }}$, given the background reconstructed top quark mass template, $P_{b}\left(m_{t, k}^{\text {reco }}\right)$, and the signal reconstructed top quark mass template with a top quark mass of $\mathrm{M}_{\text {top }}$ and energy scale shift $\Delta_{\mathrm{JES}}$, $P_{s}\left(m_{t, k}^{\mathrm{reco}} ; \mathrm{M}_{\mathrm{top}}, \Delta_{\mathrm{JES}}\right)$.

The second term, $\mathcal{L}_{\text {shape }}^{m_{j j}}$ shape, is sensitive primarily to the value of $\Delta_{\mathrm{JES}}$. It reflects the product of probabilities for each of $C_{i}$ ways of assigning the two $W$ daughter jets in each event, where $C_{i}=1,3,6$ for 2, 1, 0 tags, respectively. The probabilities are analogous to those in the first term, but are defined using the dijet mass template parameterizations.

The third term in the likelihood, $\mathcal{L}_{\text {nev }}$, represents the information arising from the number of signal and background events in the top quark mass and dijet mass samples, which are correlated. Since the number of expected signal and background events in the $W \rightarrow j j$ sample are $n_{s}$ and $n_{b}$, the expected numbers of signal and background events in the $m_{t}^{\text {reco }}$ sample are given by $\epsilon_{s} n_{s}$ and $\epsilon_{b} n_{b}$, respectively. This term expresses the likelihood associated with observing $N$ and $N^{\chi^{2}}$ events in the two samples given the expected number of events as defined above. We introduce the variables $N_{s}$ and $N_{b}$, the (un- known) number of signal and background events actually in our sample. We sum over the possible values of these two variables that are consistent with the total number of observed events. Thus the first two factors express the Poisson probability to observe $N_{s}$ signal and $N_{b}$ background events given Poisson means of $n_{s}$ and $n_{b}$, respectively. For the final factor we introduce and sum over the possible values of $N_{s}^{\chi^{2}}$ and $N_{b}^{\chi^{2}}$, variables for the (unknown) actual number of signal and background events remaining after the $\chi^{2}$ cut. The summand is the binomial probability to observe $N_{s}^{\chi^{2}}$ signal events and $N_{b}^{\chi^{2}}$ background events in the $m_{t}^{\text {reco }}$ sample given the assumed numbers of observed events $N_{s}$ and $N_{b}$ in the $m_{j j}$ sample and the $\chi^{2}$ cut efficiencies.

Finally, in the fourth term, $\mathcal{L}_{\text {bg }}$, the background normalization is constrained in the likelihood fit by a Gaussian probability distribution centered at $n_{b}^{0}$ and with width $\sigma_{n_{b}}$. The background normalizations are constrained for the 2tag, 1-tag(T), and 1-tag(L) samples. For the 0-tag subsample, no background normalization estimate is available, so no background constraint is used. Both $n_{s}$ and $n_{b}$ are required to be greater than zero.

As described in Secs. III B and III C independent detector calibrations and studies of other processes allow us to independently determine the jet energy scale, and this information is used in the reconstruction of the data events and in the determination of the signal templates. We include in the likelihood fit the knowledge of this independent jet energy calibration through an additional term in the overall likelihood:

$$
\mathcal{L}_{\mathrm{JES}}=\exp \left(-\frac{\left(\Delta_{\mathrm{JES}}-\Delta_{\mathrm{JES}}^{0}\right)^{2}}{2 \sigma_{\Delta_{\mathrm{JES}}}^{2}}\right)=\exp \left(-\frac{\Delta_{\mathrm{JES}}^{2}}{2}\right),
$$

where the simplification arises because, by our definition of $\Delta_{\mathrm{JES}}$, the calibrated shift in energy scale is $\Delta_{\mathrm{JES}}^{0}=0$ and the uncertainty is $\sigma_{\Delta_{\mathrm{JES}}}=1.0$.

The total likelihood is given by the product of the likelihoods for the four subsamples and the jet energy scale constraint:

$$
\mathcal{L}=\mathcal{L}_{2-\operatorname{tag}} \times \mathcal{L}_{1-\operatorname{tag}(\mathrm{T})} \times \mathcal{L}_{1-\operatorname{tag}(\mathrm{L})} \times \mathcal{L}_{0-\operatorname{tag}} \times \mathcal{L}_{\mathrm{JES}}
$$

The top quark pole mass $\mathrm{M}_{\text {top }}$ and the jet energy scale shift $\Delta_{\text {JES }}$ are shared between the four likelihoods. The likelihood is maximized with respect to all ten parameters $\left(n_{s}\right.$ and $n_{b}$ for four subsamples, $\Delta_{\mathrm{JES}}$, and $\mathrm{M}_{\mathrm{top}}$ ) using the MINUIT package. A likelihood curve as a function of $\mathrm{M}_{\text {top }}$ is found by maximizing the likelihood with respect to all other parameters for a series of fixed $\mathrm{M}_{\text {top }}$. The statistical uncertainty from the fit procedure is taken from the points $\mathrm{M}_{\text {top }}^{+}$and $\mathrm{M}_{\text {top }}^{-}$where the log-likelihood changes by $-1 / 2$ unit from its maximum. The positive and negative uncer- 
tainties are then scaled to achieve unit pull widths as described in Sec. V C.

\section{Method check}

The method described above is checked for any possible systematic biases by running large numbers of "pseudoexperiments," where we create, using Monte Carlo simulation, samples of signal and background events with an assumed value of the top quark mass and jet energy scale and with the same statistical properties as our observed sample. We then perform likelihood fits to each pseudoexperiment and characterize the accuracy of the technique in determining the correct values.

For each pseudoexperiment, the procedure is first to determine the number of signal and background events, then to generate a reconstructed top quark mass and dijet mass for each event, and finally to fit the resulting pseudodata using our standard machinery. The number of background events in each subsample is Poisson fluctuated around the central value given in Table II. The central value for the 0 -tag background is estimated by subtracting the estimated number of 0 -tag signal events from the observed number of 0 -tag events in the data. The number of signal events is Poisson fluctuated around the number observed in the data, minus the central value for the background expectation, for each subsample. For each event, reconstructed masses $m_{t}^{\text {reco }}$ and $m_{j j}$ are selected at random from the templates corresponding to signal or background processes. Some of the events are eliminated from the $m_{t}^{\text {reco }}$ sample, according to the $\chi^{2}$ cut efficiencies given in Table III. The resulting list of reconstructed masses is fit using exactly the same machinery used on the data, described in Sec. VB. Although this default procedure does not model correlations among the $m_{t}^{\text {reco }}$ and $m_{j j}$ values in each event, a separate check showed a complete modeling of the correlations to have a negligible effect on the measurement described here.

For each pseudoexperiment, the likelihood fit provides a measured top quark mass $\mathrm{M}_{\text {top }}$ and jet energy scale shift $\Delta_{\mathrm{JES}}$, as well as positive and negative errors $(\delta+$ and $\delta-$ )
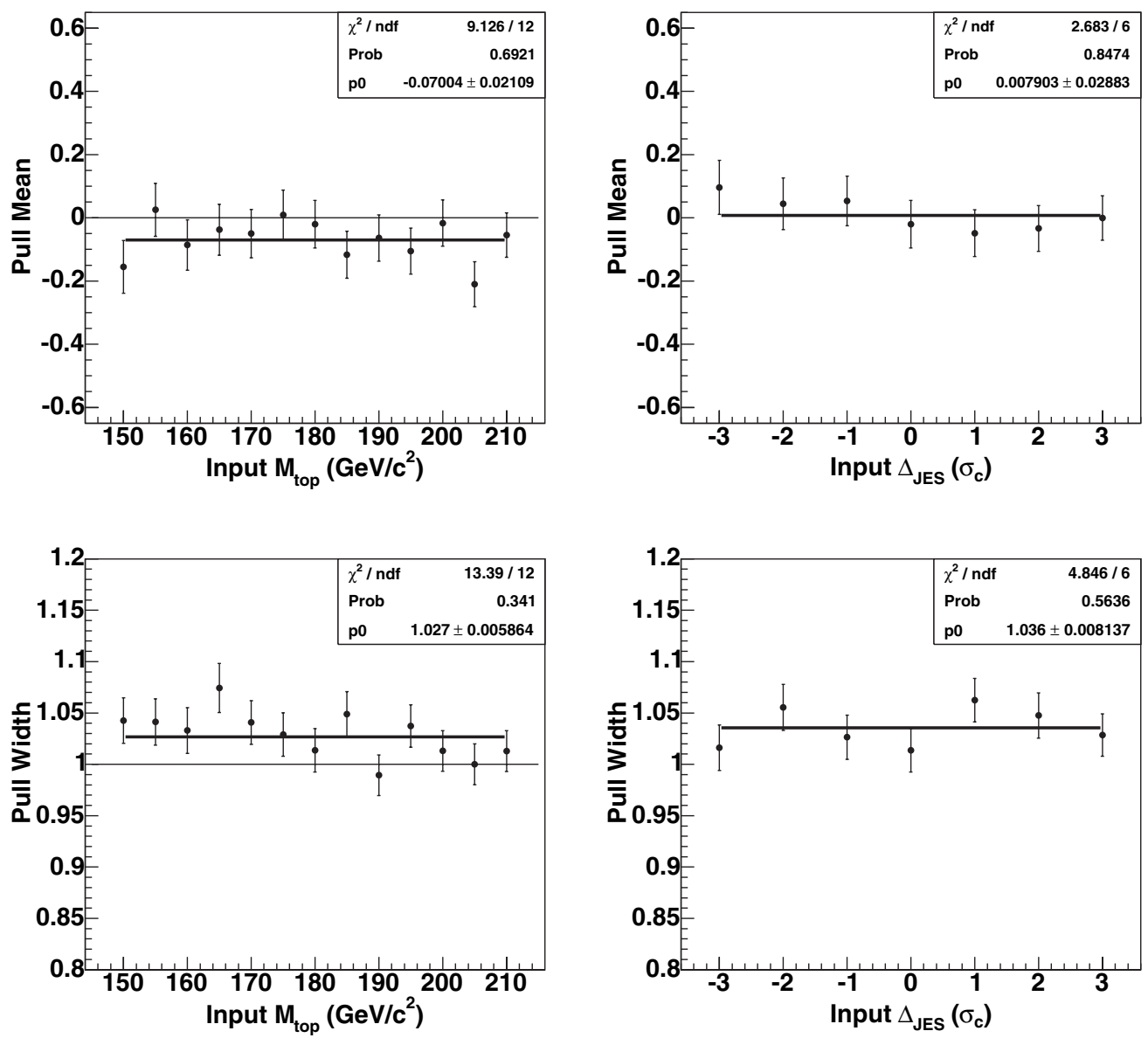

FIG. 16. The mean (top) and width (bottom) of pull distributions from sets of 2500 pseudoexperiments are shown. On the left, the jet energy scale is fixed at its nominal value, and the generated top quark mass is varied from $150 \mathrm{GeV} / c^{2}$ to $210 \mathrm{GeV} / c^{2}$. On the right, the top quark mass is fixed at $180 \mathrm{GeV} / c^{2}$, and the input jet energy scale shift is varied from $-3 \sigma_{c}$ to $+3 \sigma_{c}$. The error bars come mostly from the limited statistics of the Monte Carlo samples from which the pseudodata is taken. 
for each from the $\Delta \ln L=-1 / 2$ procedure. We check the pull distribution for $\mathrm{M}_{\mathrm{top}}$, defined using a symmetrized uncertainty on the top quark mass as the distribution of $\left(\mathrm{M}_{\text {top }}-\mathrm{M}_{\text {top }}^{\text {input }}\right) / 0.5\left(\delta_{\mathrm{M}_{\text {top }}}^{+}+\delta_{\mathrm{M}_{\text {top }}}^{-}\right)$, where $\mathrm{M}_{\text {top }}^{\text {input }}$ is the generated top quark mass. A pull distribution is generated for each of 12 input values for the top quark mass, keeping $\Delta_{\text {JES }}$ fixed to zero, where 2500 pseudoexperiments are generated for each input mass value, and each pull distribution is fitted using a Gaussian function. We determine a similar set of pull distributions for various values of the $\Delta_{\mathrm{JES}}$ parameter, keeping the top quark mass fixed to $180 \mathrm{GeV} / c^{2}$, although the results in this case are correlated since they all use the same Monte Carlo event sample. The mean and sigma of the fitted functions are shown in Fig. 16. Defining the pull distributions using the reported asymmetric uncertainties does not systematically change the results.

In the pull distributions as a function of top quark mass, the pull means show a small offset for this particular slice of the $\mathrm{M}_{\mathrm{top}}-\Delta_{\mathrm{JES}}$ plane. Since the value of this offset varies with location in the $\mathrm{M}_{\mathrm{top}}-\Delta_{\mathrm{JES}}$ space, instead of directly correcting $\mathrm{M}_{\text {top }}$ we take the average offset of $0.3 \mathrm{GeV} / c^{2}$ as a systematic uncertainty on the measurement.

In addition the pull widths are slightly larger than 1 due to the modest statistics of the event sample. For the expected number of events with current luminosity, and for templates such as the ones described in Sec. VA, the resulting likelihood curve is typically non-Gaussian, and in fact, typically shallower than Gaussian. The pull distributions become more Gaussian (with width one) as pseudoexperiments with more events are performed. With 10 times the statistics, the pull widths are consistent with unity.

For the current data sample, the quoted measurement with uncertainties is designed to have pull width equal to one by scaling the errors taken from $\Delta \ln L=-1 / 2$. The scale factor is the pull width from the lower left plot of Fig. 16, averaged over the values of top quark mass, giving 1.027.

\section{RESULTS ON THE DATA}

We fit the events in the data using the procedure described in Sec. V. After detailing the results, we present several additional results performed as cross-checks on the primary measurement.

\section{A. Subsample likelihood curves}

The likelihood fit is first performed on each subsample separately. In these fits, each subsample likelihood contains the $\Delta_{\text {JES }}$ constraint term given in Eq. (5.8). For a series of $\mathrm{M}_{\mathrm{top}}$ and $\Delta_{\mathrm{JES}}$ values, the corresponding parameters are fixed, while the likelihood is maximized with
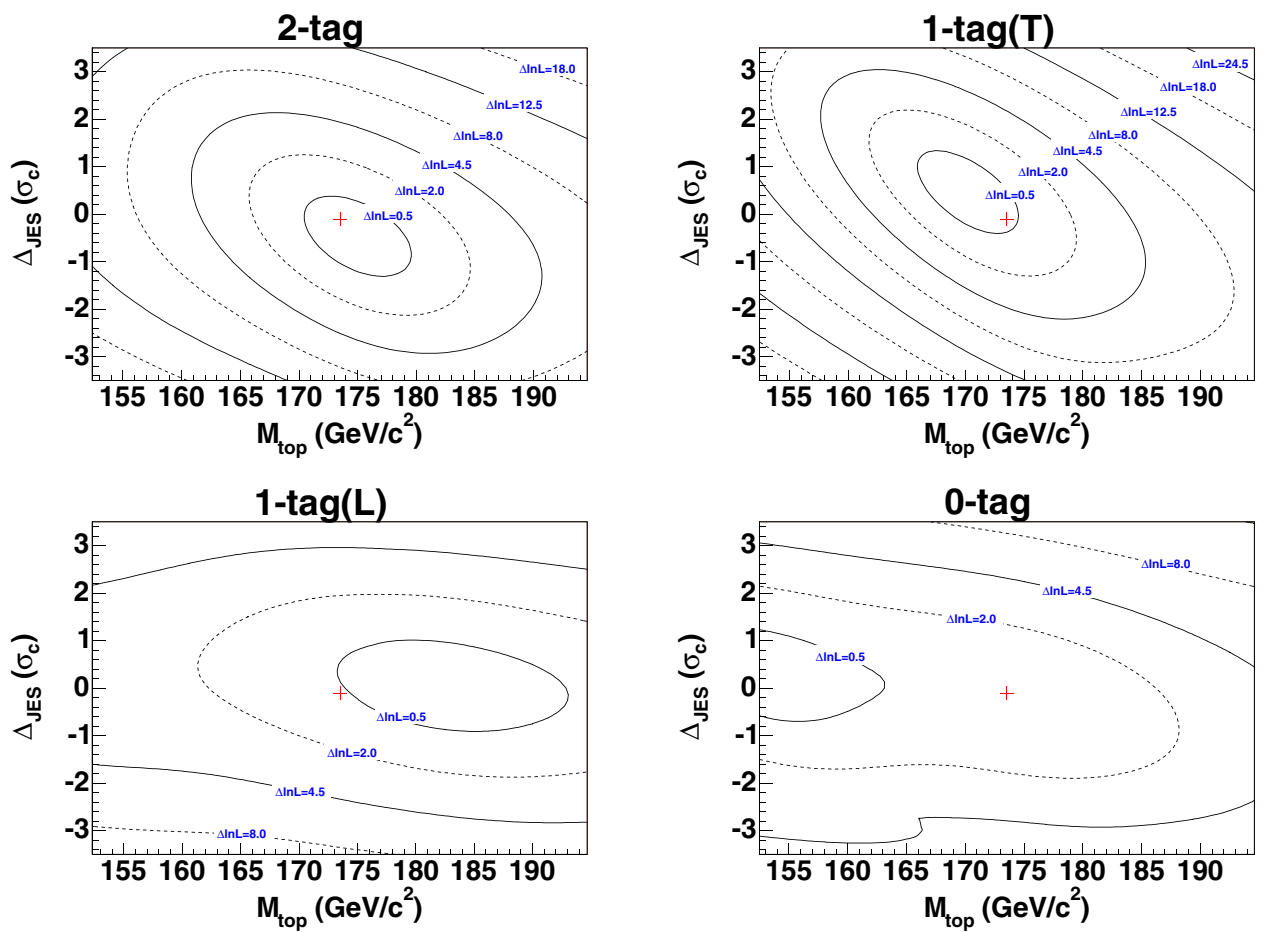

FIG. 17 (color online). The contours of the likelihood in the $\mathrm{M}_{\mathrm{top}}-\Delta_{\mathrm{JES}}$ plane for the independent fit to each subsample in the data. At each point in the plane, the likelihood is maximized with respect to the other free parameters. A crosshair shows the maximum likelihood point from the combined fit, and contours are given at regular intervals in $\Delta \ln L$, the change in log-likelihood from its maximum. Upper left: 2-tag events; upper right: 1-tag(T) events; lower left: 1-tag(L) events; lower right: 0-tag events. 


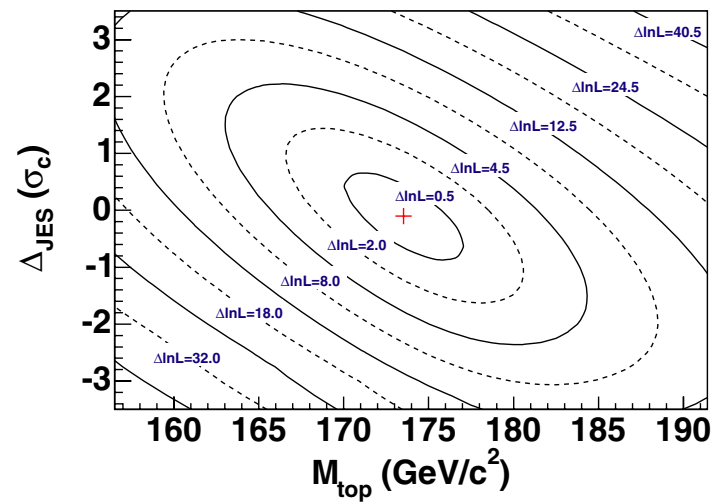

FIG. 18 (color online). The contours of the likelihood in the $\mathrm{M}_{\text {top }}-\Delta_{\mathrm{JES}}$ plane for the combined fit to all four subsamples. At each point in the plane, the likelihood is maximized with respect to the other free parameters. The crosshair shows the best fit point, and contours are given at regular intervals in $\Delta \ln L$, the change in log-likelihood from its maximum.

TABLE VI. The input constraints and fitted values are given for all free parameters in the combined likelihood fit.

\begin{tabular}{|c|c|c|c|c|c|}
\hline Category & & 2-tag & 1-tag(T) & 1-tag(L) & 0 -tag \\
\hline $\mathrm{M}_{\mathrm{top}}$ & $\begin{array}{c}\text { constr. } \\
\text { fit }\end{array}$ & \multicolumn{4}{|c|}{ 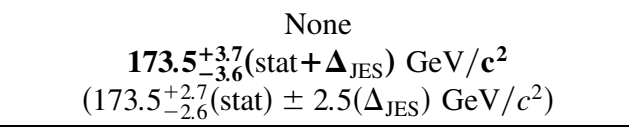 } \\
\hline$\Delta_{\mathrm{JES}}$ & $\begin{array}{l}\text { constr. } \\
\text { fit } \\
\text { constr. }\end{array}$ & \multicolumn{4}{|c|}{$\begin{array}{l}0.0 \pm 1.0 \sigma_{c} \\
-0.10_{-0.80}^{+0.78} \sigma_{c} \\
\quad \text { None }\end{array}$} \\
\hline & fit & $23.5 \pm 5.0$ & $53.9 \pm 7.9$ & $14.3 \pm 5.2$ & $28.3 \pm 8.3$ \\
\hline$n_{b}^{W}$ & $\begin{array}{c}\text { constr. } \\
\text { fit }\end{array}$ & $\begin{array}{c}1.89 \pm 0.52 \\
1.8 \pm 0.5\end{array}$ & $\begin{array}{c}10.4 \pm 1.72 \\
10.1 \pm 1.7\end{array}$ & $\begin{array}{c}14.3 \pm 2.45 \\
15.5 \pm 2.2\end{array}$ & $\begin{array}{c}\text { None } \\
15.7_{-7.1}^{+8.0}\end{array}$ \\
\hline
\end{tabular}

respect to the remaining parameters $\left(n_{s}\right.$ and $\left.n_{b}\right)$ using MINUIT. The resulting likelihood contours in the $\mathrm{M}_{\mathrm{top}}-\Delta_{\mathrm{JES}}$ plane are shown in Fig. 17.

\section{B. Results of combined likelihood}

Finally, the likelihood is maximized with respect to all parameters using all four subsamples. The result, after scaling the $\Delta(\ln L)=-1 / 2$ errors as described in Sec. V C, is a top quark mass of $173.5_{-3.6}^{+3.7} \mathrm{GeV} / c^{2}$. The simultaneous measurement of the jet energy scale shift is $-0.10_{-0.80}^{+0.78} \sigma_{c}$. The correlation between the $\mathrm{M}_{\text {top }}$ and $\Delta_{\mathrm{JES}}$ fits is -0.676 . The combined likelihood as a function of $\mathrm{M}_{\text {top }}$ and $\Delta_{\text {JES }}$ is shown in Fig. 18. For each value of $\mathrm{M}_{\text {top }}$ and $\Delta_{\mathrm{JES}}$, the likelihood is maximized with respect to all other parameters. This likelihood is not the simple product of the four likelihoods shown in Fig. 17 because the $\Delta_{\text {JES }}$ constraint term $\mathcal{L}_{\text {JES }}$ is included in each of the subsample fits, but of course only once in the combined fit.

The uncertainty on $M_{\text {top }}$ from the likelihood fit is a combination of the statistical uncertainty in extracting a measurement of $\mathrm{M}_{\text {top }}$ and the systematic uncertainty due to allowed variations of $\Delta_{\mathrm{JES}}$. It is possible to get an idea of the size of each contribution. Fixing $\Delta_{\mathrm{JES}}$ to its fitted value of $-0.10 \sigma_{c}$ fitting for $\mathrm{M}_{\text {top }}$ alone yields a top quark mass measurement of $173.5_{-2.6}^{+2.7}$ (stat) $\mathrm{GeV} / c^{2}$, corresponding to the "pure statistical" uncertainty. Subtracting this uncertainty in quadrature from the full uncertainty gives an $\mathbf{M}_{\text {top }}$ uncertainty due to the jet energy scale of $\pm 2.5 \mathrm{GeV} / c^{2}$.

The input constraints and fit results for the combined fit are given in Table VI. Figure 19 shows the consistency of the reconstructed top quark mass distribution in each sub-

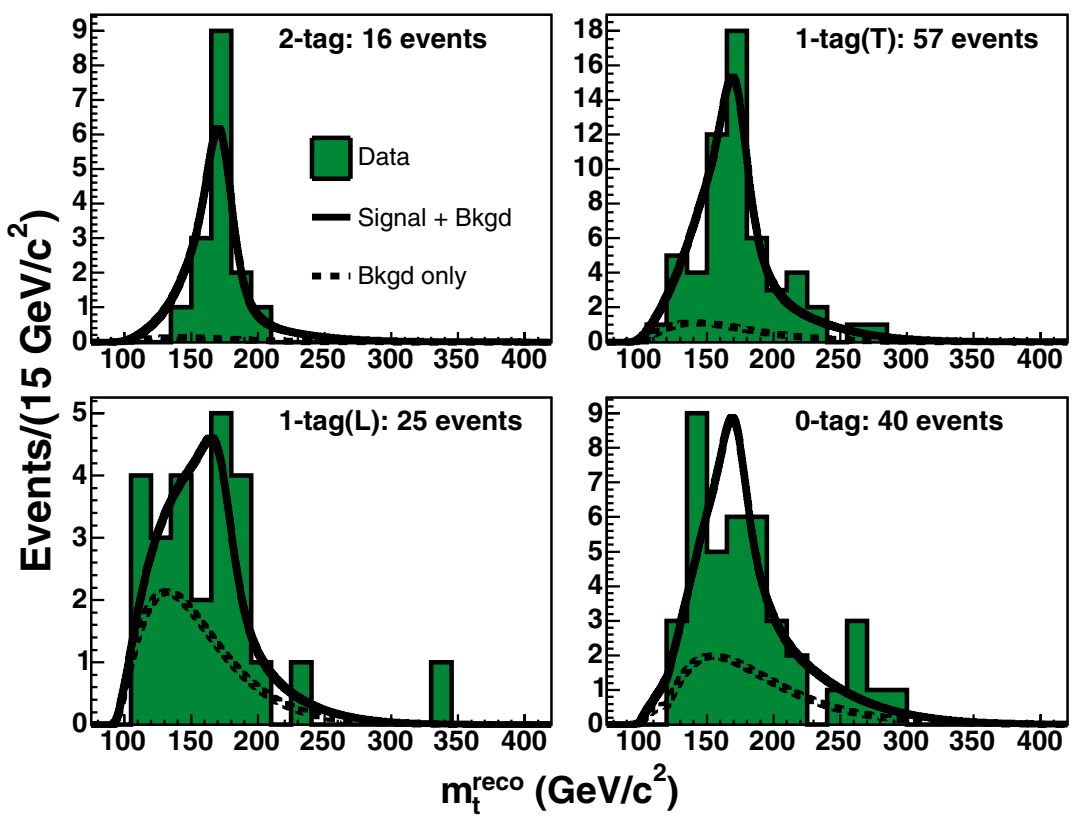

FIG. 19 (color online). The reconstructed top quark mass distribution for each subsample is shown overlaid with the expected distribution using the top quark mass, jet energy scale shift, signal normalization, and background normalization from the combined fit. 


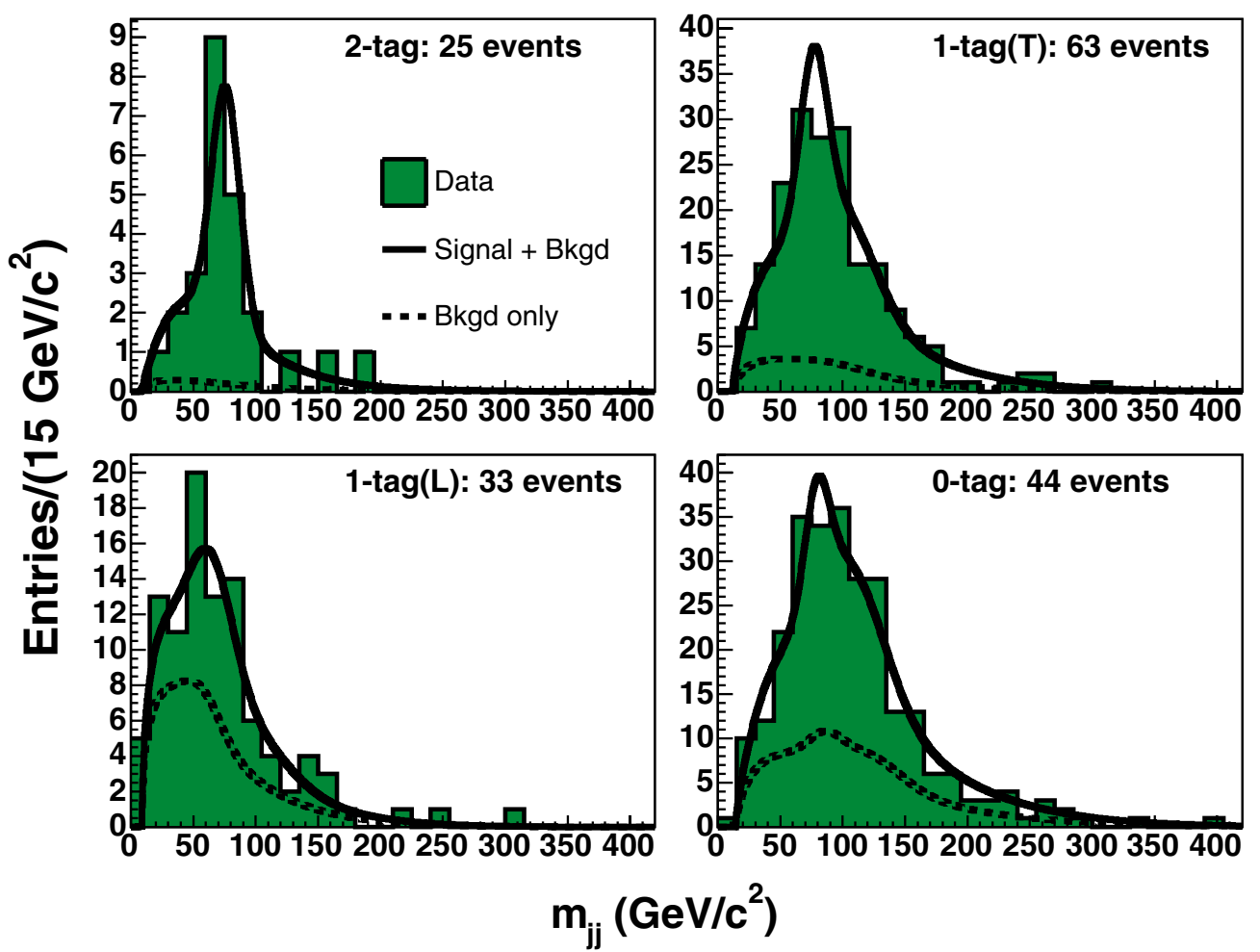

FIG. 20 (color online). The reconstructed dijet mass distribution for each subsample is shown overlaid with the expected distribution using the top quark mass, jet energy scale, signal normalization, and background normalization from the combined fit.

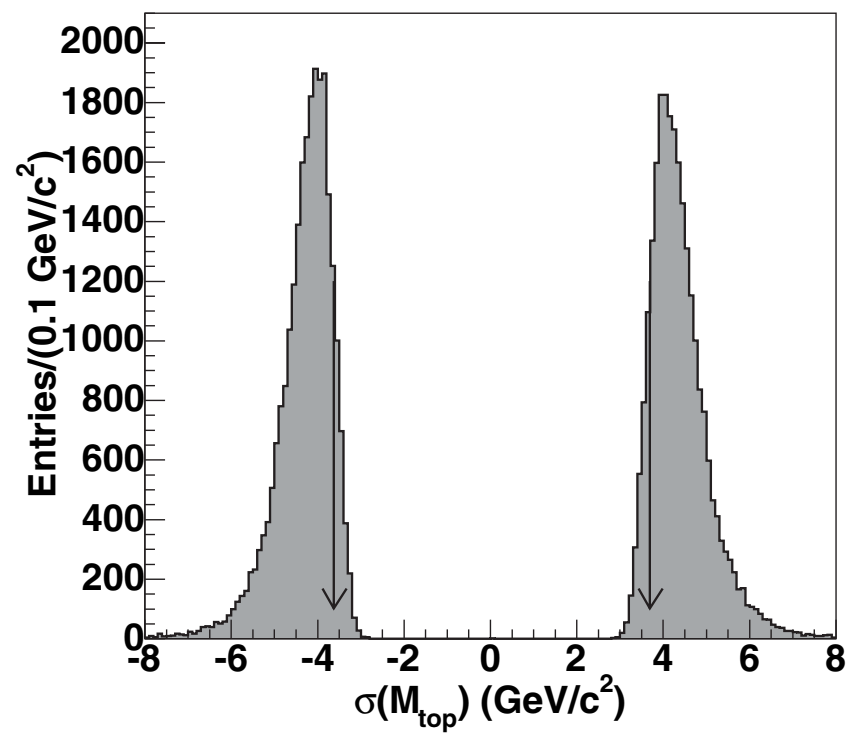

FIG. 21. The distributions of positive and negative uncertainties from the likelihood fit are shown, for pseudoexperiments generated with a top quark mass of $172.5 \mathrm{GeV} / c^{2}$, the nominal jet energy scale, and the number of events in each subsample as observed in the data. Arrows indicate the positive and negative uncertainties from the likelihood fit to the data; $9.2 \%$ of the pseudoexperiments have smaller uncertainties. sample with the combined fit results, while Fig. 20 shows the same for the reconstructed dijet mass.

A set of pseudoexperiments is generated with a top quark mass of $172.5 \mathrm{GeV} / c^{2}$ (close to the central value from the fit), the nominal jet energy scale, and with the number of events in each subsample equal to the number observed in our data (Table I). In Fig. 21, the positive and negative uncertainties from the likelihood fits are plotted. Arrows indicate the uncertainties from the fit to the data. Although smaller than the median uncertainties from the pseudoexperiments, the uncertainties on the data are reasonable $-9.2 \%$ of the pseudoexperiments have smaller uncertainties than those returned by the fit to the data. The distributions do not change significantly if a top quark mass value of $165 \mathrm{GeV} / c^{2}$ or $180 \mathrm{GeV} / c^{2}$ is used. The better-than-expected uncertainties are consistent with the sharpness of the reconstructed top quark mass peaks in the 2-tag and 1-tag(T) subsamples, as shown in Fig. 19.

\section{Alternate fits}

In addition to the primary result described above, a number of additional fits are performed as cross-checks and to investigate the effect of certain assumptions on our measurement. The differences between the primary fit and the alternate fits are briefly described below, along with the resulting top quark mass measurements. Table VII summa- 
TABLE VII. The results of alternate fits are summarized. For the cases that do not include the jet energy scale systematic effect in the likelihood fit result, the independently determined systematic is given for comparison (see Sec. VIID for more details).

\begin{tabular}{lcc}
\hline \hline Method & $\mathrm{M}_{\text {top }}$ fit result & $\Delta_{\text {JES }}$ fit result \\
\hline & {$\left[\mathrm{GeV} / c^{2}\right]$} & {$\left[\sigma_{c}\right]$} \\
Default & $173.5_{-3.6}^{+3.7}\left(\mathrm{stat}+\Delta_{\mathrm{JES}}\right)$ & $-0.10_{-0.80}^{+0.78}$ \\
No $\Delta_{\text {JES }}$ constr. & $174.0 \pm 4.5\left(\right.$ stat $\left.+\Delta_{\mathrm{JES}}\right)$ & $-0.25 \pm 1.22$ \\
$\mathrm{M}_{\text {top }}$-only & $173.2_{-2.8}^{+2.9}($ stat $) \oplus 3.1\left(\Delta_{\mathrm{JES}}\right)$ & $\mathrm{N} / \mathrm{A}$ \\
$+\mathrm{JPB}$ & $173.0_{-2.8}^{+2.9}($ stat $) \oplus 3.0\left(\Delta_{\mathrm{JES}}\right)$ & $\mathrm{N} / \mathrm{A}$ \\
\hline \hline
\end{tabular}

rizes the results. The results from the alternate methods are quite similar, though the methods are highly correlated.

\section{Fit without $\Delta_{\mathrm{JES}}$ constraint}

In the primary fit, the measurement of $\Delta_{\mathrm{JES}}$ is treated as an update to the extrinsic calibration by including the Gaussian constraint $\mathcal{L}_{\text {JES }}$ in the likelihood. Here the $\mathcal{L}_{\text {JES }}$ term is removed, so that all the jet energy scale information comes from the in situ calibration to the resonance of the hadronically decaying $W$ boson. The resulting top quark mass measurement is $174.0 \pm 4.5\left(\right.$ stat $\left.+\Delta_{\mathrm{JES}}\right) \mathrm{GeV} / c^{2}$, and the simultaneous fit for $\Delta_{\mathrm{JES}}$ gives $-0.25 \pm$ 1.22 (stat) $\sigma_{c}$. Although the systematic uncertainties are not explicitly evaluated for this approach, they are not expected to be significantly different from those of the primary analysis.

\section{Traditional $\mathrm{M}_{\mathrm{top}}$-only fit}

For this alternate result, the traditional fit for a single variable, $\mathrm{M}_{\mathrm{top}}$, is performed using a single reconstructed quantity, $m_{t}^{\text {reco }}$. This fit is virtually identical to the analysis performed in run I [5]. The event selection and $m_{t}^{\text {reco }}$ reconstruction are exactly as described earlier. With only one reconstructed quantity and one measured quantity, the template parameterizations are simpler. Signal and background p.d.f.'s for $m_{t}^{\text {reco }}$ are fitted without any dependence on $\Delta_{\mathrm{JES}}$ but otherwise identical in form to those described above. The form of the likelihood used is also much simpler, since the term $\mathcal{L}_{\text {shape }}^{m_{j j}}$ is absent and $\mathcal{L}_{\text {nev }}$ is greatly simplified with only the sample of events after the $\chi^{2}$ cut used. For each subsample, the likelihood is given by
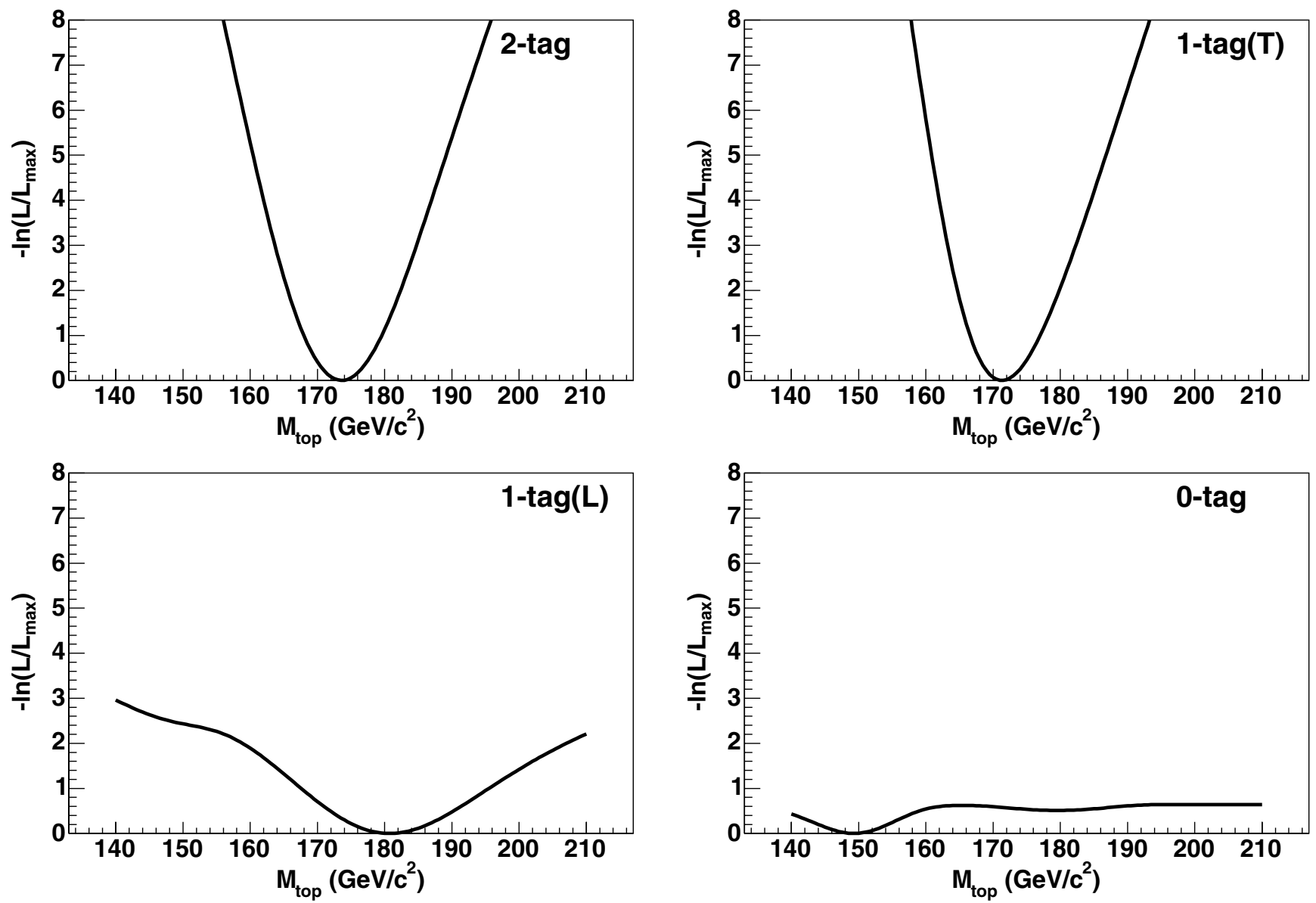

FIG. 22 (color online). The negative log-likelihood curves as a function of the top quark mass are shown for the $\mathrm{M}_{\text {top }}$-only fit to each subsample in the data. Upper left: 2-tag events; upper right: 1-tag(T) events; lower left: 1-tag(L) events; lower right: 0-tag events. 


$$
\begin{aligned}
\mathcal{L}= & \operatorname{Pois}\left(N ; n_{s}+n_{b}\right) \\
& \times \prod_{k=1}^{N} \frac{n_{s} P_{s}\left(m_{t, k}^{\mathrm{reco}} ; \mathrm{M}_{\mathrm{top}}\right)+n_{b} P_{b}\left(m_{t, k}^{\mathrm{reco}}\right)}{n_{s}+n_{b}} \\
& \times \exp \left(-\frac{\left(n_{b}-n_{b}^{0}\right)^{2}}{2 \sigma_{n_{b}}^{2}}\right)
\end{aligned}
$$

where in this context $n_{s}, n_{b}$, and $N$ refer to the number of events expected and observed in the sample after the $\chi^{2}$ cut. The combined likelihood is simply the product of the subsample likelihoods.

The fitted value of the top quark mass using this method is $173.2_{-2.8}^{+2.9}$ (stat) $\mathrm{GeV} / c^{2}$, with a central value very close to the result from the primary measurement. For this result, of course, since the jet energy scale systematic uncertainty is not accounted for in the likelihood fit, its effect on the top quark mass uncertainty must be estimated separately and added in quadrature. The negative log-likelihood curves for the $\mathbf{M}_{\text {top }}$-only fit in each subsample are shown in Fig. 22. As can be seen in the lower-right panel, the 0-tag subsample contributes very little to the overall measurement. This is because the fit prefers a small signal contribution in this sample with no background constraint, which results in very little sensitivity to $\mathrm{M}_{\mathrm{top}}$.

\section{Traditional $\mathrm{M}_{\mathrm{top}}$-only fit with additional tag category}

Events with two $b$ tags carry the most information about the top quark mass because of their high purity and narrow reconstructed mass templates. In this alternate analysis, we increase the number of events with two $b$ tags by allowing one of the tags to come from the jet probability tagger. We establish a new category of events with exactly one secondary vertex tag and an additional JPB tag; the former requirement ensures that these events do not overlap with the 2-tag subsample. The events in this category are then removed from the 1-tag(T) and 1-tag(L) samples so that all the subsamples remain disjoint. Eighteen events are found in this category in the data sample: 4 out of 18 events were newly categorized from 1-tag(L) and 14 events from 1$\operatorname{tag}(\mathrm{T})$ in the default configuration.

The expected backgrounds in the new event category are estimated to be $0.52 \pm 0.26$ events from $W b \bar{b}, W c$, and $W c \bar{c}$ processes, $0.15 \pm 0.08$ events from non- $W$ background, $0.38 \pm 0.19$ events from mistagged $W+$ jets events, $0.08 \pm 0.04$ events from single top, and $0.05 \pm$ 0.03 events from the diboson processes $W W$ and $W Z$. The total number of background events is thus estimated to be $1.2 \pm 0.6$ for the new subsample. The background estimates for the exclusive one-tag subsamples change to account for the reduced acceptances.

The likelihood used to extract the top quark mass from this data is that described above in Sec. VIC 2, i.e. using a $\mathrm{M}_{\mathrm{top}}$-only fit. Figure 23 shows the reconstructed top quark mass distribution for the events with one secondary vertex

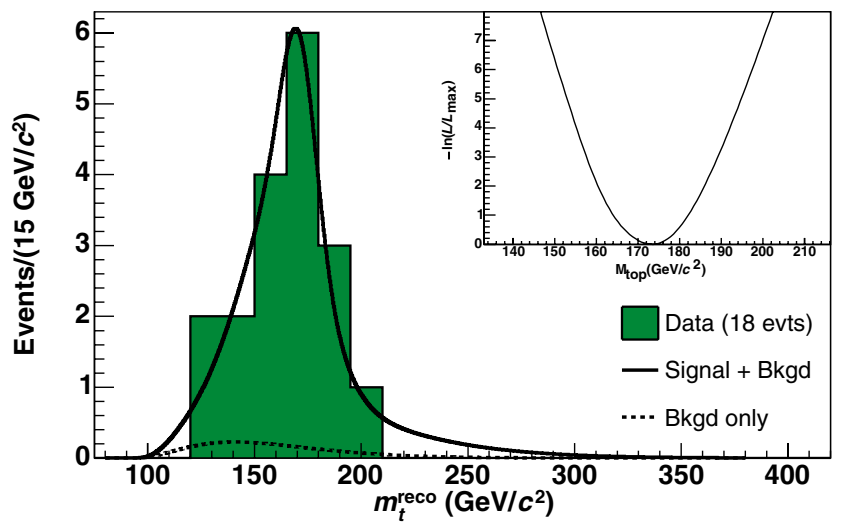

FIG. 23 (color online). The reconstructed top quark mass distribution for the 18 events with one secondary vertex tag and one JPB tag, overlaid with the expected distribution from the fit to this subsample. The inset shows the shape of $-\Delta \log \mathcal{L}$ for the fit to these events as a function of the top quark mass.

tag and one JPB tag, along with the expected distribution using parameters taken from the fit to only this set of events. The inset shows the negative log-likelihood curve for this subsample alone. Using only the 18 events in this subsample, the measured top quark mass is $173.3_{-6.5}^{+6.1}$ (stat) $\mathrm{GeV} / c^{2}$.

This result using this data sample can be combined with the other four categories of events. A sensitivity study shows that the combined likelihood including the new class of events improves the expected statistical uncertainty by $2.6 \%$. In addition to the statistical improvement, increasing the number of double-tagged events improves the jet en-

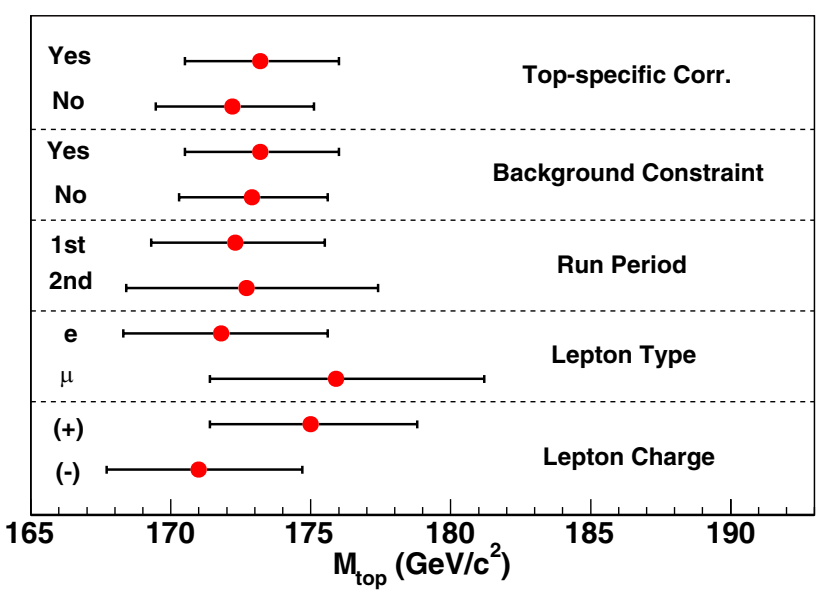

FIG. 24 (color online). Top quark mass measurements using the $\mathrm{M}_{\text {top}}$-only fit are compared for different assumptions [topspecific corrections (default) vs generic jet out-of-cone corrections, constrained backgrounds vs unconstrained] and different ways of subdividing the sample (two different run periods, electrons vs muons, positive-charge leptons vs negative-charge leptons). All results with only statistical errors are consistent. 


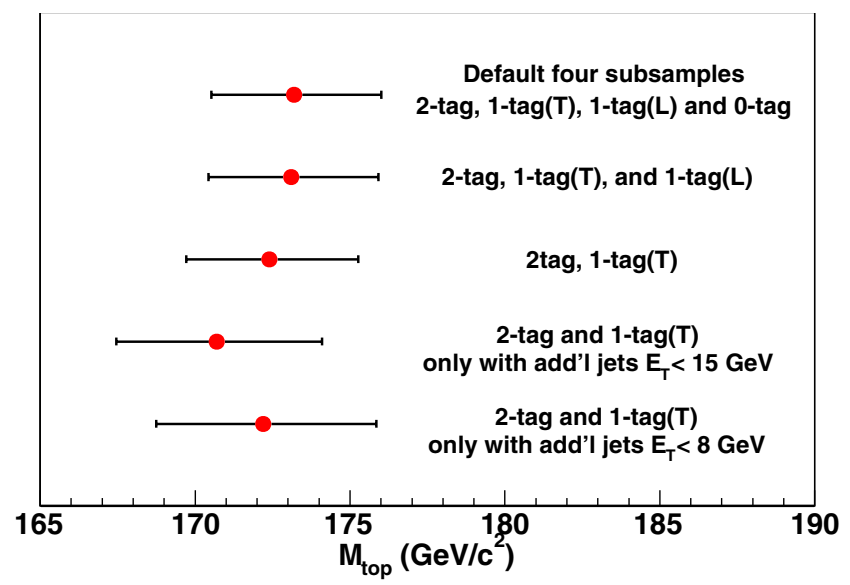

FIG. 25 (color online). Top quark mass measurements using the $\mathrm{M}_{\text {top }}$-only fit are compared for various samples. From top to bottom: all four subsamples; 2-tag, 1-tag(T), and 1-tag(L) subsamples; 2-tag and 1-tag(T) subsamples only; 2-tag and 1-tag(T) subsamples only, additional jets $E_{T}<15 \mathrm{GeV}$; 2-tag and 1$\operatorname{tag}(\mathrm{T})$ subsamples only, additional jets $E_{T}<8 \mathrm{GeV}$. All results with only statistical errors are found to be consistent.

ergy systematic uncertainty. The resulting combined top quark mass measurement on the five subsamples is $173.0_{-2.8}^{+2.9}$ (stat) $\mathrm{GeV} / c^{2}$.

\section{Cross-checks on the results}

The measurement of the top quark mass is checked by performing the analysis in various subsamples and with different jet corrections and background normalization constraints in order to ensure the robustness of the result. In these cross-checks, we use the traditional $\mathrm{M}_{\text {top }}$-only fit described in Sec. VIC 2 for simplicity.

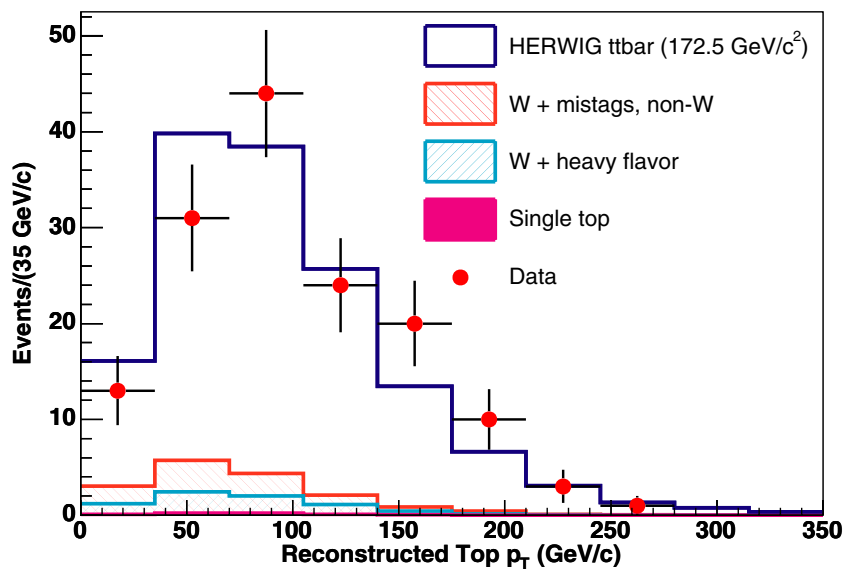

FIG. 26 (color online). The $p_{T}$ distribution of the reconstructed top quarks for 73 signal candidate events [2-tag and $1-\operatorname{tag}(\mathrm{T})$ subsamples], compared to the prediction from HERWIG $t \bar{t}$ signal events (with generated top quark mass of $172.5 \mathrm{GeV} / c^{2}$ ) and simulated background events.

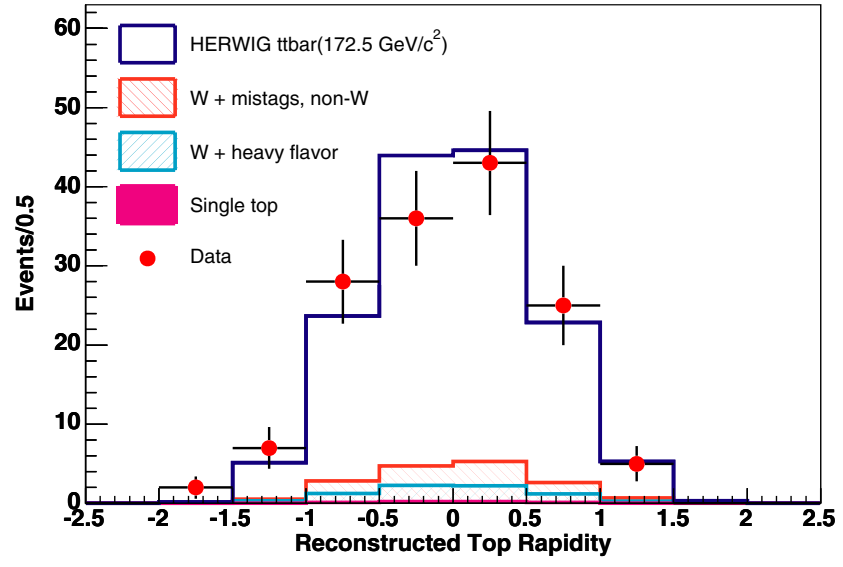

FIG. 27 (color online). The rapidity distribution of the reconstructed top quarks for 73 signal candidate events [2-tag and 1$\operatorname{tag}(\mathrm{T})$ subsamples] compared to the prediction from HERWIG $t \bar{t}$ signal events (with generated top quark mass of $172.5 \mathrm{GeV} / c^{2}$ ) and simulated background events.

Figure 24 shows the resulting top quark mass measurement for various modifications to the method. Any inconsistencies would most likely indicate problems with the detector or the analysis method. First the measurement using top-specific corrections (the default) and using generic out-of-cone jet corrections is compared. Next fits using the background constraints (the default) and without the constraints are shown. For the remaining comparisons, the data set is divided into two subsamples. First results are shown from two different run periods, then events with a primary electron vs those with a primary muon, and finally positive-charge primary leptons vs negative-charge primary leptons. Except in the case of the generic jet corrections, the default reconstructed mass templates are used.

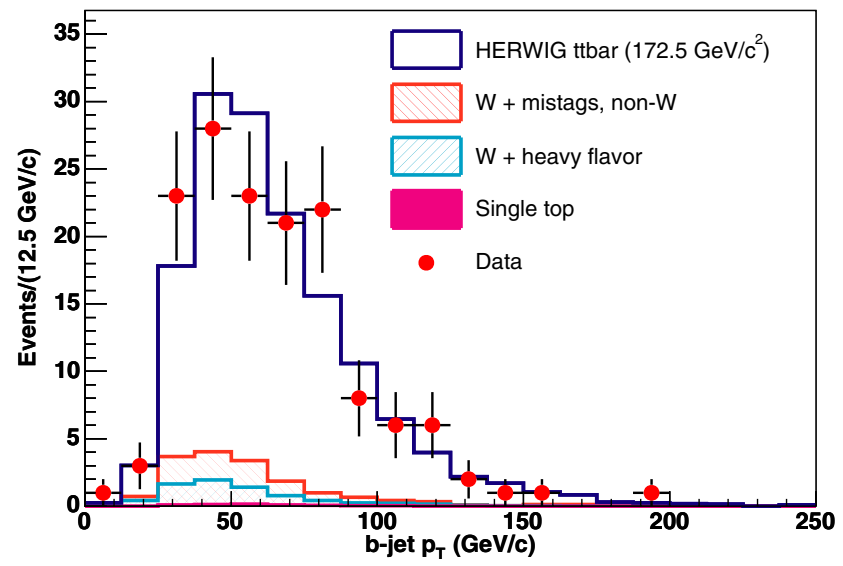

FIG. 28 (color online). The $p_{T}$ distribution of the reconstructed $b$ jets for 73 signal candidate events [2-tag and 1$\operatorname{tag}(\mathrm{T})$ subsamples] compared to the prediction from HERWIG $t \bar{t}$ signal events (with generated top quark mass of $172.5 \mathrm{GeV} / c^{2}$ ) and simulated background events. 


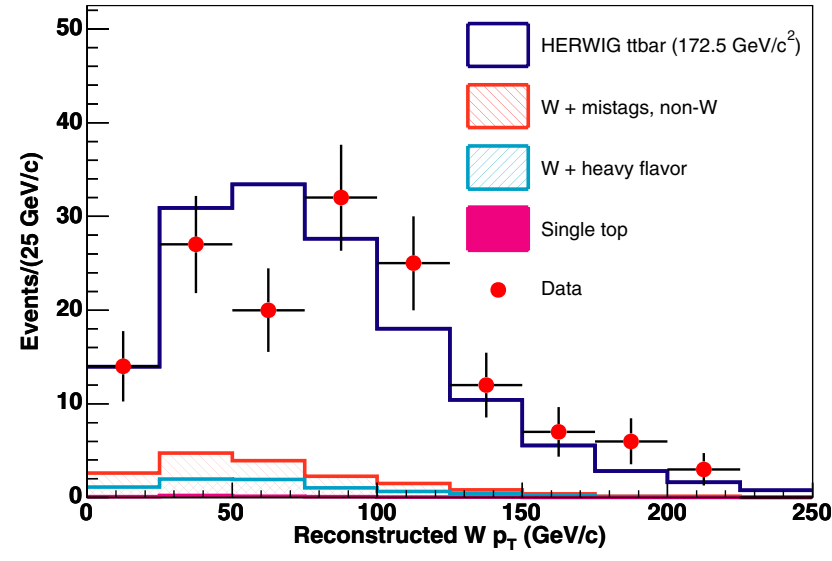

FIG. 29 (color online). The $p_{T}$ distribution of the reconstructed $W$ bosons for 73 signal candidate events [2-tag and 1$\operatorname{tag}(\mathrm{T})$ subsamples] compared to the prediction from HERWIG $t \bar{t}$ signal events (with generated top quark mass of $172.5 \mathrm{GeV} / c^{2}$ ) and simulated background events.

All the results are consistent with each other and with the primary measurement.

A series of top quark mass measurements using different subsamples of the data is shown in Fig. 25. The primary effects of the increasingly tight selection are, first, to increase the sample purity and thereby decrease sensitivity to modeling of the background processes; and second, to select events with low extra jet activity, decreasing sensitivity to modeling of ISR and FSR. The list of samples used is as follows from top to bottom: all four subsamples (default, 138 events after $\chi^{2}$ cut); 2-tag, 1-tag(T), and 1$\operatorname{tag}(\mathrm{L})$ only (98 events); 2 -tag and 1-tag(T) only (73 events); 2-tag and 1-tag(T) only with any additional jets required to have $E_{T}<15 \mathrm{GeV}$ (56 events); 2-tag and 1$\operatorname{tag}(\mathrm{T})$ only with any additional jets required to have $E_{T}<$

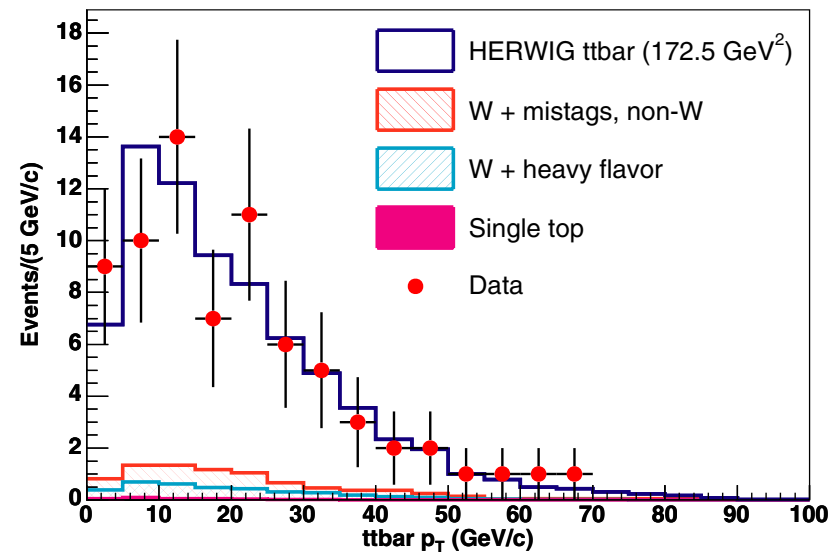

FIG. 30 (color online). The $p_{T}$ distribution of the reconstructed $t \bar{t}$ system for 73 signal candidate events [2-tag and 1$\operatorname{tag}(\mathrm{T})$ subsamples] compared to the prediction from HERWIG $t \bar{t}$ signal events (with generated top quark mass of $172.5 \mathrm{GeV} / c^{2}$ ) and simulated background events.

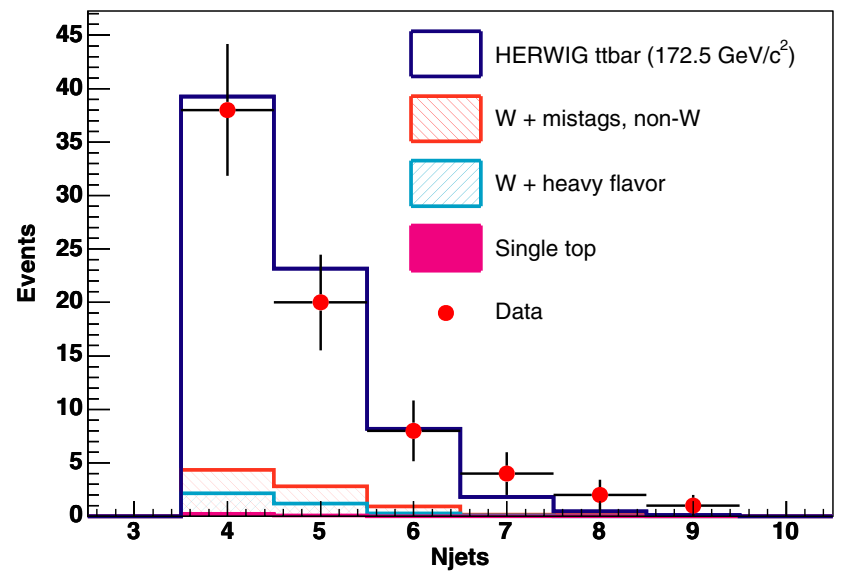

FIG. 31 (color online). The number of jets distribution for 73 signal candidate events [2-tag and 1-tag(T) subsamples] compared to the prediction from HERWIG $t \bar{t}$ signal events (with generated top quark mass of $172.5 \mathrm{GeV} / c^{2}$ ) and simulated background events. Jets are required to have $E_{T}>8 \mathrm{GeV}$ and $|\eta|<2.0$.

$8 \mathrm{GeV}$ (38 events). For the last two cases, top mass templates are prepared with the additional requirements. Again we find that all results are consistent, indicating that background kinematics and extra jet activity are reasonably well modeled.

\section{E. Kinematic distributions}

We compare various kinematic distributions for the $t \bar{t}$ signal candidate events with the Monte Carlo predictions for combined signal and backgrounds. Comparisons of kinematic distributions tell us how well the Monte Carlo models the data, which is very important in this kinematic analysis. This information could additionally be used to test whether the kinematic properties of the top quark we observe are consistent with standard model predictions. For these distributions, we use only 2-tag and 1-tag(T) events with $\chi^{2}<9$ (73 events), in order to increase the signal purity. All kinematic quantities are defined using the output of the $\chi^{2}$ fitter, so that both jet-quark assignments and the $p_{T}$ of each object are taken at the minimum $\chi^{2}$ point.

Figures 26 and 27 show the $p_{T}$ and rapidity distributions of the reconstructed top quarks, respectively. The data distributions are in agreement with predictions using HERWIG $t \bar{t}$ signal events with top quark mass of $172.5 \mathrm{GeV} / c^{2}$ and simulated background events.

We also find good agreement in the $p_{T}$ distribution of the $b$ jets from top decays, shown in Fig. 28. Good modeling of the $b$-jet spectrum by the Monte Carlo simulation is one of the most important things for a good determination of the top quark mass. Figure 29 shows the $p_{T}$ distribution of the reconstructed $W$ bosons. 
The $p_{T}$ distribution of the $t \bar{t}$ system is shown in Fig. 30, which has good agreement between the data and the prediction from simulated events. This distribution is sensitive to the modeling of initial-state radiation. The distribution of the number of jets from data events is also compared with the prediction from the Monte Carlo simulation, as shown in Fig. 31. To be counted in this plot, each jet is required to have $E_{T}>8 \mathrm{GeV}$ and $|\eta|<2.0$; note that this distribution is sculpted by the selection requirements for the 2-tag and 1-tag $(\mathrm{T})$ subsamples. The data and prediction are in good agreement, indicating that the number of extra jets (from hard initial and final-state radiation) is reasonably well modeled by HERWIG.

\section{SYSTEMATIC UNCERTAINTIES}

Systematic uncertainties arise from uncertainties in our understanding of the detector response and in the assumptions employed to infer a top quark mass from the observed data. The magnitudes of such uncertainties are estimated using auxiliary data collected for this purpose and large samples of Monte Carlo simulated events that allow us to estimate the sensitivity of the measurements to reasonable variations in analysis assumptions.

For each source of systematic uncertainty, the relevant quantities or parameters are varied by $\pm 1 \sigma$, and new $178 \mathrm{GeV} / c^{2} t \bar{t}$ signal and background Monte Carlo templates are produced by performing event selection and mass reconstruction on the modified samples. Events for pseudoexperiments (see Sec. VC) are taken from these new templates, but the signal and background p.d.f.'s used in the analysis remain unchanged. The shift in the median fitted top quark mass for a large ensemble of pseudoexperiments is taken as the systematic uncertainty associated with a given assumption or effect. When the uncertainty on a given systematic shift due to the statistics of the Monte Carlo sample is larger than the shift itself, that statistical uncertainty is used for the systematic uncertainty.

\section{A. Systematic uncertainties related to $\Delta_{\text {JES }}$ measurement}

The use of the observed $W$ boson mass to constrain the jet energy scale calibration essentially measures the average energy response of light-quark jets arising from the decay of the colorless $W$ boson. However, the top quark mass also depends on the energy response to $b$ quark jets. This introduces three possible sources of uncertainty: (i) uncertainties in energy response arising from uncertainties in the decay properties of bottom quarks, (ii) uncertainties arising from the imperfect knowledge of the fragmentation properties of bottom quarks, and (iii) uncertainties in energy response arising from the different color flow associated with bottom quark jets produced in top quark decay.
We varied the $B$ meson semileptonic branching ratios by about $10 \%$ of their values, corresponding to their measurement uncertainties [28], in our Monte Carlo models to estimate the size of this uncertainty in the overall energy scale of the bottom quark jet. We found that this introduced an additional uncertainty in the bottom quark jet energy scale of $0.4 \%$, resulting in an uncertainty in the extraction of the top quark mass of $0.4 \mathrm{GeV} / c^{2}$. We used the highstatistics measurements of bottom quark fragmentation observed in $Z \rightarrow b \bar{b}$ decays at the LEP and SLC colliders to constrain the fragmentation models in our Monte Carlo calculations. We found that this variation introduced an additional top quark mass uncertainty of $0.4 \mathrm{GeV} / c^{2}$. In order to test the effects of possible variations in energy response due to different models of "color flow" in the top quark production and decay, we varied the parameters of the algorithms used to generate this color flow in both HERWIG and PYTHIA and conservatively estimated that this could result in an uncertainty in the bottom quark jet energy scale of $0.3 \%$. This results in an additional uncertainty in the top quark mass of $0.3 \mathrm{GeV} / c^{2}$.

We add these three contributions in quadrature and include an additional $0.6 \mathrm{GeV} / c^{2}$ systematic uncertainty in the top quark mass arising from the modeling of the bottom quark jets.

In this analysis, the jet energy scale shift parameter $\Delta_{\mathrm{JES}}$ is assumed to have the same value for all jets in all events. However, the jet energy systematics have contributions from many sources, and those component uncertainties in general have different dependence on, for example, jet $p_{T}$ and $\eta$, or on the event environment. To estimate the uncertainty arising from the assumption of a monolithic jet energy scale, we produce samples in which the components of the jet energy systematics are shifted independently and in various combinations. The typical shift in the

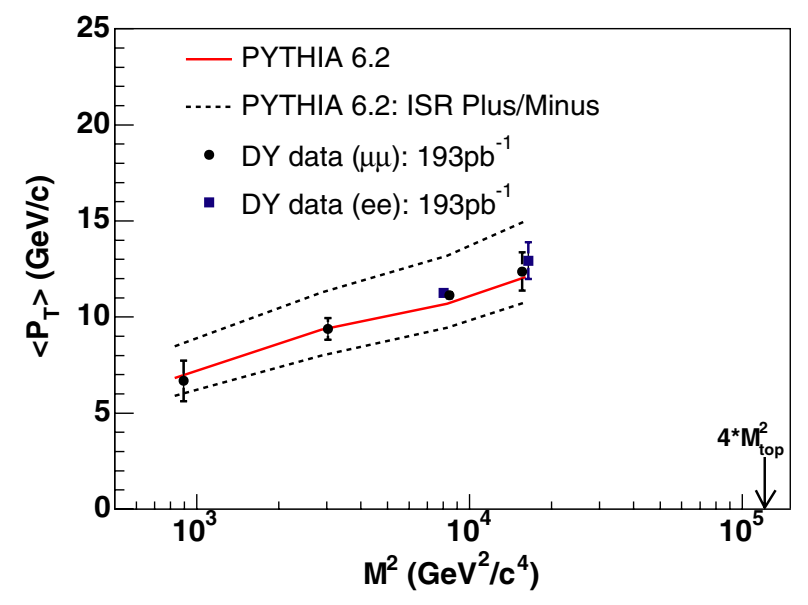

FIG. 32 (color online). The average $p_{T}$ of the dilepton system, which corresponds to the level of ISR activity, shows a logarithmic dependence on the dilepton invariant mass $M_{l l}^{2}$. The data are compared with the predictions of PYTHIA 6.2 and of the $+1 \sigma_{\text {ISR }}$ and $-1 \sigma_{\text {ISR }}$ samples. 
top quark mass measurement is $0.5 \mathrm{GeV} / c^{2}$, which is taken as the largest part of a "method" systematic. This systematic includes the offset of $0.07 \sigma$ observed in the pull distributions of Fig. 16, which translates to $0.3 \mathrm{GeV} / c^{2}$.

Finally, the effect of the jet energy scale uncertainty on background events must be treated separately, since it is not included in the background template parameterization. We find a small uncertainty on $\mathrm{M}_{\text {top }}, 0.04 \mathrm{GeV} / c^{2}$, which we add linearly to the uncertainty due to the overall jet energy scale shift since the effects are correlated.

\section{B. ISR/FSR/PDF systematic errors}

The systematic uncertainties due to initial-state radiation, final-state radiation, and parton density functions are summarized in this section.

Extra jets originating from the incoming partons and outgoing partons affect the measurement of $\mathbf{M}_{\text {top }}$ when they are misidentified as jets from the final-state partons or change the kinematics of the final-state partons. ISR and FSR are controlled by the same DGLAP evolution equation that tells us the probability for a parton to branch [3034]. ISR is studied using Drell-Yan events in dilepton channels. The advantage of Drell-Yan events is that there is no FSR, and they are produced by the $q \bar{q}$ annihilation process, as are most $(\sim 85 \%) t \bar{t}$ pairs.

The level of ISR is measured as a function of the DrellYan mass scale and shows a logarithmic dependence on the Drell-Yan mass squared, as shown in Fig. 32. By extrapolation, the ISR effect is then estimated at top pair production energies. Based on this measurement, two ISR systematic Monte Carlo samples $\left(+1 \sigma_{\text {ISR }}\right.$ and $\left.-1 \sigma_{\text {ISR }}\right)$ are produced using PYTHIA, by varying the value of $\Lambda_{\mathrm{QCD}}$ and scale factor, $K$ to the transverse momentum scale for ISR showering. The parameters used are $\Lambda_{\mathrm{QCD}}(5$ flavors $)=292 \mathrm{MeV}, K=0.5$ for $+1 \sigma_{\text {ISR }}$ and $\Lambda_{\mathrm{QCD}}(5$ flavors $)=73 \mathrm{MeV}, K=2.0$ for $-1 \sigma_{\mathrm{ISR}}$. The corresponding curves of Drell-Yan dilepton $\left\langle p_{T}\right\rangle$ vs invariant mass squared are shown in Fig. 32. Although ISR is also sensitive to the choice of parton distribution function (PDF), the PDF uncertainty is not included as a part of the ISR uncertainty. Because a PDF change affects not only ISR but also hard scattering kinematics, the PDF uncertainty is treated separately. The largest top quark mass shift between default PYTHIA and the two ISR samples, $0.4 \mathrm{GeV} / c^{2}$, is taken as the ISR uncertainty.

Since ISR and FSR shower algorithms are the same, the same variations in $\Lambda_{\mathrm{QCD}}$ and $K$ are used to generate FSR systematic samples by varying a set of parameters specific to FSR modeling. The largest top quark mass shift between default PYTHIA and the two FSR samples, $0.6 \mathrm{GeV} / c^{2}$, is used as the FSR uncertainty. We examine the effects of higher order corrections to $t \bar{t}$ production using MC@NLO [35], a full NLO Monte Carlo. Based on distributions of the number of jets and the $t \bar{t} p_{T}$, we find that NLO effects are covered by the ISR/FSR systematics.
The calculation of the top quark invariant mass does not depend directly on the choice of input PDF. However, changing the PDF changes the top quark $\eta$ and $p_{T}$ distributions as well as the size of ISR. This results in a change in the jet $p_{T}$ distributions and in the probability of selecting the correct jets, both of which affect the reconstructed top quark mass.

To examine the systematic effect due to PDF uncertainties, 20 pairs of uncertainty sets based on CTEQ6M are used [36,37]. These PDFs provide “ $\pm 1 \sigma$ " variations for 20 independent eigenvectors but do not include variation in $\Lambda_{\mathrm{QCD}}$. In addition, the MRST group [38] provides PDFs with different assumptions for the value of $\Lambda_{\mathrm{QCD}}$. The difference between the measured top quark mass using the MRST72 $\left(\Lambda_{\mathrm{QCD}}=228 \mathrm{MeV}\right)$ and MRST75 $\left(\Lambda_{\mathrm{QCD}}=\right.$ $300 \mathrm{MeV}$ ) PDFs is taken as an uncertainty, as is the difference between leading order PDFs CTEQ5L and MRST72. Instead of 43 different, fully simulated sets of events, a single simulated sample is used, and mass templates are generated for the different PDF sets by weighting events according to the probability of observing their incoming partons using each PDF set. This technique also removes most of the uncertainty due to limited Monte Carlo statistics. A symmetrized uncertainty for each of the 20 pairs of CTEQ6 PDFs (determined by varying one eigenvector at a time) is added in quadrature to get one part of the PDF uncertainty, $0.20 \mathrm{GeV} / c^{2}$. An additional systematic error of $0.22 \mathrm{GeV} / c^{2}$ comes from the variation of $\Lambda_{\mathrm{QCD}}$. This is consistent with the much less precise estimate using fully simulated samples. Adding a negligible contribution from the CTEQ-MRST difference, the total PDF uncertainty comes to $0.3 \mathrm{GeV} / c^{2}$.

In order to check the sensitivity of the top quark mass measurement to a very different top quark $p_{T}$ distribution due to a new physics process, we have used a signal Monte Carlo sample with resonant $t \bar{t}$ production, where the resonance occurs at $700 \mathrm{GeV} / c^{2}$ and then top quarks decay according to the standard model. The measured top quark mass is shifted by only $1.5 \mathrm{GeV} / c^{2}$, demonstrating that this kinematic top mass fitter is nearly insensitive to the $p_{T}$ of the top quark.

\section{Other systematic errors}

The remaining sources of systematic uncertainty are described in this section.

The difference in the top quark mass between HERWIG and PYTHIA samples is $0.2 \pm 0.2 \mathrm{GeV} / c^{2}$. To be conservative, this difference is taken as another systematic uncertainty, although the differences in ISR and FSR between the two generators are already taken into account in the ISR and FSR uncertainties, and fragmentation effects are accounted for in the jet energy uncertainties.

The largest uncertainty in the shape of the reconstructed mass templates for background events is due to the uncertainty in the $Q^{2}$ scale that is used for the calculation of the 
TABLE VIII. The uncertainties on the $\mathrm{M}_{\text {top }}$-only top quark mass measurement are shown for each jet energy systematic error. Estimates are obtained for the independent subsamples as well as for the combined measurement.

Jet energy systematic: $\Delta \mathrm{M}_{\mathrm{top}}\left(\mathrm{GeV} / c^{2}\right)$

2-tag 1-tag(T) 1-tag(L) 0-tag Combined

Response relative to central

$\begin{array}{lllll}0.6 & 0.6 & 0.6 & 1.0 & 0.6\end{array}$

Modeling hadron jets (absolute scale)

$\begin{array}{lllll}2.0 & 2.3 & 2.0 & 2.7 & 2.2\end{array}$

Modeling out-of-cone energy and underlying event

$\begin{array}{lllll}2.2 & 2.2 & 1.9 & 1.9 & 2.1\end{array}$

Total systematic due to jet energies

3.0

$$
3.2
$$$$
2.8
$$

3.4

3.1

hard scattering and for the shower evolution. Different background shapes are obtained for four different $Q^{2}$ scales $\left(4 M_{W}^{2}, \quad M_{W}^{2}, M_{W}^{2} / 4\right.$, and $\left.M_{W}^{2}+P_{T W}^{2}\right)$ using ALPGEN MC samples. An ALPGEN $W b \bar{b}+2$ parton Monte Carlo sample is used for the tagged events and a $W+4$ parton sample for the 0 -tag and mistagged events. Half of the largest difference in top quark mass from pseudoexperiments using these samples is used as the systematic uncertainty, $0.4 \mathrm{GeV} / c^{2}$. Smaller contributions to the background shape uncertainty are estimated by performing sets of pseudoexperiments in which background events are drawn not from the combined background template but from templates for one of the individual background processes, or from the templates derived from QCD-enriched data. Half of the largest difference observed in these pseudoexperiments is $0.3 \mathrm{GeV} / c^{2}$ for the different background processes, and

TABLE IX. Summary of all systematic uncertainties for the combined analysis and two alternate fits.

\begin{tabular}{|c|c|c|c|c|}
\hline \multirow[t]{2}{*}{ Method } & \multicolumn{2}{|c|}{ Primary } & \multirow{2}{*}{$\begin{array}{c}\mathrm{M}_{\text {top }} \text {-only } \\
\Delta \mathrm{M}_{\mathrm{top}} \\
\left(\mathrm{GeV} / c^{2}\right)\end{array}$} & \multirow{2}{*}{$\begin{array}{c}\mathrm{M}_{\text {top }} \text {-only } \\
+\mathrm{JPB} \\
\Delta \mathrm{M}_{\mathrm{top}} \\
\left(\mathrm{GeV} / \mathrm{c}^{2}\right)\end{array}$} \\
\hline & $\begin{array}{c}\Delta \mathrm{M}_{\mathrm{top}} \\
\left(\mathrm{GeV} / c^{2}\right)\end{array}$ & $\begin{array}{c}\Delta\left(\Delta_{\mathrm{JES}}\right) \\
\left(\sigma_{c}\right)\end{array}$ & & \\
\hline Jet energy & N/A & N/A & 3.1 & 3.0 \\
\hline$b$-jet energy & 0.6 & 0.25 & 0.6 & 0.6 \\
\hline Method & 0.5 & 0.02 & N/A & N/A \\
\hline ISR & 0.4 & 0.08 & 0.4 & 0.3 \\
\hline FSR & 0.6 & 0.06 & 0.4 & 0.6 \\
\hline PDFs & 0.3 & 0.04 & 0.4 & 0.4 \\
\hline Generators & 0.2 & 0.15 & 0.3 & 0.2 \\
\hline Bkgd shape & 0.5 & 0.08 & 0.5 & 0.5 \\
\hline$b$ tagging & 0.1 & 0.01 & 0.2 & 0.3 \\
\hline MC stats & 0.3 & 0.05 & 0.4 & 0.4 \\
\hline Total & 1.3 & 0.33 & 3.3 & 3.2 \\
\hline
\end{tabular}

$0.1 \mathrm{GeV} / c^{2}$ for the different models of the QCD background. Both of these are taken as additional systematic uncertainties on the top quark mass due to background shape modeling.

Different $b$-tagging efficiency in data and simulation can introduce a bias in the top quark mass measurement. The $E_{T}^{\mathrm{jet}}$ dependence of the $b$ tagging in data and simulation agree very well. But if a slope on the $E_{T}^{\text {jet }}$ dependence (consistent at $1 \sigma$ with the measurement) is introduced in the tagging efficiency, the shift in the top quark mass is $0.1 \mathrm{GeV} / c^{2}$, which is taken as a systematic uncertainty.

The analysis can have a systematic bias due to the finite statistics of Monte Carlo samples that are used to obtain the signal and background shape parameterizations. For a rough estimate of this uncertainty, sets of pseudoexperiments are performed with a series of fluctuated signal and background templates; in each fluctuated template, each bin is varied randomly according to Poisson statistics. For each fluctuated template, the median top quark mass measured by pseudoexperiments is shifted. The typical shift due to these statistical fluctuations, taken as a systematic uncertainty due to Monte Carlo statistics, is $0.3 \mathrm{GeV} / c^{2}$.

\section{Jet systematic errors}

The systematics on jet energy measurements are described in detail in Sec. III B. The primary analysis fits for the jet energy scale, and the error from the likelihood fit includes a contribution due to these systematics. For the $\mathrm{M}_{\text {top }}$-only fits, however, this systematic uncertainty must be estimated independently.

To determine the systematic uncertainty on the top quark mass measurement given the various sources of uncertainty on the jet energy measurements, the mass shifts for $+1 \sigma_{c}$ and $-1 \sigma_{c}$ perturbations in the jet energies are extracted, and a symmetric uncertainty for each source is defined as half the difference between the two shifts. Table VIII lists the uncertainties obtained for the $\mathrm{M}_{\text {top }}$-only measurement. The total systematic uncertainty in the top quark mass due to jet energy measurements is $3.1 \mathrm{GeV} / c^{2}$ for the combined measurement. The corresponding systematic uncertainties for an independent measurement in each subsample are listed for comparison.

\section{E. Total systematic uncertainty}

The systematic uncertainties for the combined fit are listed in Table IX. The total systematic uncertainty is estimated to be $1.3 \mathrm{GeV} / c^{2}$, exclusive of the uncertainty due to jet energy scale that is included in the likelihood error. Also shown in Table IX are the systematic uncertainties on the $\Delta_{\text {JES }}$ measurement $\left(0.33 \sigma_{c}\right.$ total $)$, and the systematic uncertainties on $\mathrm{M}_{\text {top }}$ for the $\mathrm{M}_{\text {top }}$-only measurement without $\left(3.3 \mathrm{GeV} / c^{2}\right.$ total $)$ and with (3.2 GeV/c $c^{2}$ total) JPB tags. 


\section{CONCLUSION}

We have made a new measurement of the top quark mass,

$$
\begin{aligned}
& 173.5_{-3.6}^{+3.7}\left(\text { stat }+\Delta_{\mathrm{JES}}\right) \pm 1.3 \text { (other syst) } \mathrm{GeV} / c^{2} \\
& =173.5_{-3.8}^{+3.9} \mathrm{GeV} / c^{2}
\end{aligned}
$$

using a novel technique that utilizes the jet energy scale information provided by the hadronically decaying $W$ boson in the top quark events. This new top quark mass measurement provides the most precise single measurement on this important physical parameter. We have performed a cross-check of this result using a more traditional fit that does not use the in situ jet energy scale information and found excellent agreement in the central value of the top quark mass: $173.2_{-2.8}^{+2.9}$ (stat) \pm 3.3 (syst) $\mathrm{GeV} / c^{2}$. Finally, by adding an algorithm to increase the number of tagged $b$ jets, we measure: $173.0_{-2.8}^{+2.9}$ (stat) \pm 3.2(syst) $\mathrm{GeV} / c^{2}$.

This measurement is part of a rich top physics program at CDF. As the luminosity acquired increases from the current $318 \mathrm{pb}^{-1}$ to an expected $4000-7000 \mathrm{pb}^{-1}$ for run II, the statistical uncertainty on the top quark mass will improve. Using our technique, the dominant systematic uncertainty on the measurement, associated with the jet energy scale, also will be reduced with more data. As we approach total uncertainties of approximately $2.0 \mathrm{GeV} / c^{2}$, the uncertainties due to initial and final-state radiation, as well as the bottom quark jet energy scale, become comparable to the statistical uncertainties associ- ated with the top quark mass and jet energy scale measurement. We expect that these other systematics also can be improved with more work and more data in the relevant control samples. Additional top quark mass results from $\mathrm{CDF}$ are expected in the near future. We expect that these will continue to provide important inputs into our understanding of the fundamental fermions and the nature of the electroweak interaction.

\section{ACKNOWLEDGMENTS}

We thank the Fermilab staff and the technical staffs of the participating institutions for their vital contributions. This work was supported by the U.S. Department of Energy and National Science Foundation; the Italian Istituto Nazionale di Fisica Nucleare; the Ministry of Education, Culture, Sports, Science and Technology of Japan; the Natural Sciences and Engineering Research Council of Canada; the National Science Council of the Republic of China; the Swiss National Science Foundation; the A.P. Sloan Foundation; the Bundesministerium für Bildung und Forschung, Germany; the Korean Science and Engineering Foundation and the Korean Research Foundation; the Particle Physics and Astronomy Research Council and the Royal Society, UK; the Russian Foundation for Basic Research; the Comisión Interministerial de Ciencia y Tecnología, Spain; in part by the European Community's Human Potential Programme under Contract No. HPRNCT-2002-00292; and the Academy of Finland.
[1] F. Abe et al. (CDF Collaboration), Phys. Rev. Lett. 74, 2626 (1995).

[2] S. Abachi et al. (D0 Collaboration), Phys. Rev. Lett. 74, 2632 (1995).

[3] P. Azzi et al. (TEVEWWG Collaboration), hep-ex/ 0404010.

[4] F. Abe et al. (CDF Collaboration), Phys. Rev. D 50, 2966 (1994).

[5] T. Affolder et al. (CDF Collaboration), Phys. Rev. D 63, 032003 (2001).

[6] D. Acosta et al. (CDF Collaboration), Phys. Rev. D 71, 032001 (2005).

[7] A. Sill (CDF Collaboration), Nucl. Instrum. Methods Phys. Res., Sect. A 447, 1 (2000).

[8] T. Affolder et al. (CDF Collaboration), Nucl. Instrum. Methods Phys. Res., Sect. A 526, 249 (2004).

[9] L. Balka et al. (CDF Collaboration), Nucl. Instrum. Methods Phys. Res., Sect. A 267, 272 (1988).

[10] S. Bertolucci et al. (CDF Collaboration), Nucl. Instrum. Methods Phys. Res., Sect. A 267, 301 (1988).

[11] M. G. Albrow et al. (CDF Collaboration), Nucl. Instrum. Methods Phys. Res., Sect. A 480, 524 (2002).
[12] G. Ascoli et al., Nucl. Instrum. Methods Phys. Res., Sect. A 268, 33 (1988).

[13] E. J. Thomson et al., IEEE Trans. Nucl. Sci. 49, 1063 (2002).

[14] G. Corcella, I. G. Knowles, G. Marchesini, S. Moretti, K. Odagiri, P. Richardson, M. H. Seymour, and B. R. Webber, J. High Energy Phys. 01 (2001) 010.

[15] T. Sjostrand, L. Lonnblad, and S. Mrenna, hep-ph/ 0108264.

[16] M.L. Mangano, M. Moretti, F. Piccinini, R. Pittau, and A. D. Polosa, J. High Energy Phys. 07 (2003) 001.

[17] E. A. Gerchtein and M. Paulini, eConf C0303241, TUM T005 (2003), physics/0306031.

[18] R. Brun, F. Bruyant, M. Maire, A.C. McPherson, and P. Zanarini CERN Report No. DD/EE/84-1, 1987.

[19] G. Grindhammer, M. Rudowicz, and S. Peters, Nucl. Instrum. Methods Phys. Res., Sect. A 290, 469 (1990).

[20] A. Abulencia et al. (CDF Collaboration) hep-ex/0508029.

[21] F. Abe et al. (CDF Collaboration), Phys. Rev. D 45, 1448 (1992).

[22] D. Acosta et al. (CDF Collaboration), Phys. Rev. D 71, 052003 (2005). 
[23] F. Abe et al. (CDF Collaboration), Phys. Rev. D 50, 2966 (1994).

[24] D. Buskulic et al. (ALEPH Collaboration), Phys. Lett. B 313, 535 (1993).

[25] J. M. Campbell and R. K. Ellis, Phys. Rev. D 60, 113006 (1999).

[26] B. W. Harris, E. Laenen, L. Phaf, Z. Sullivan, and S. Weinzierl, Phys. Rev. D 66, 054024 (2002).

[27] A. Bhatti et al., hep-ex/0510047.

[28] S. Eidelman et al. (Particle Data Group Collaboration), Phys. Lett. B 592, 1 (2004).

[29] S. M. Oliveira, L. Brucher, R. Santos, and A. Barroso,
Phys. Rev. D 64, 017301 (2001).

[30] Y. L. Dokshitzer, Sov. Phys. JETP 46, 641 (1977).

[31] V. N. Gribov and L. N. Lipatov, Yad. Fiz. 15, 1218 (1972).

[32] V. N. Gribov and L. N. Lipatov, Yad. Fiz. 15, 781 (1972).

[33] L. N. Lipatov, Sov. J. Nucl. Phys. 20, 94 (1975).

[34] G. Altarelli and G. Parisi, Nucl. Phys. B126, 298 (1977).

[35] S. Frixione, P. Nason, and B. Webber, J. High Energy Phys. 08 (2003) 007.

[36] J. Pumplin et al., J. High Energy Phys. 07 (2002) 012.

[37] D. Stump et al., J. High Energy Phys. 10 (2003) 046.

[38] A. D. Martin, R. G. Roberts, W. J. Stirling, and R.S. Thorne, Eur. Phys. J. C 14, 133 (2000). 